\title{
A Chirped Pulse Fourier Transform Millimeter Wave Spectrometer for Room Temperature, Gas Mixture Analysis
}

\author{
Brent Joseph Harris \\ St. Charles, MO \\ Masters of Arts, University of Arizona, 2011 \\ Bachelors of Science, University of Missouri-Columbia, 2005
}

A Dissertation presented to the Graduate Faculty of the University of Virginia in Candidacy for the Degree of

Doctor of Philosophy

Department of Chemistry

University of Virginia

May, 2014 


\begin{abstract}
A new arbitrary waveform generator based, chirped pulse spectrometer for highresolution, millimeter wave rotational spectroscopy of gas mixtures is presented. The spectrometer band covers the frequency range from $260-295 \mathrm{GHz}$, a favorable spectral window for room temperature analysis of small molecules with a molecular mass of less than 150 amu. The design leverages the state of the art in solid-state millimeter light sources and high-speed digital electronics to introduce significant advances for millimeter/submillimeter spectroscopy, which is conventionally addressed by direct absorption techniques. By efficient use of a high power millimeter wave light source, the chirped pulse spectrometer is 10,000 times faster than modern direct absorption counterparts. In addition to speed, the time resolved emission spectroscopy approach enables analytical tools that can be used to speed spectral assignment in unknown samples. Results of sensitivity, dynamic range, and chemical selectivity are presented using compressed gases as an initial benchmark followed by extensions to real gas mixtures. The results have implications to a broad set of basic science research applications and make a strong case for wider application to analytical challenges as a new commercial platform.
\end{abstract}




\section{Table of Contents}

\section{Introductory Chapter: Astrochemistry}

The origins of millimeter wave analytical chemistry

I. Chemistry in the Interstellar Medium ................................... 1

II. Chemical Synthesis and Detection by Molecular Rotational

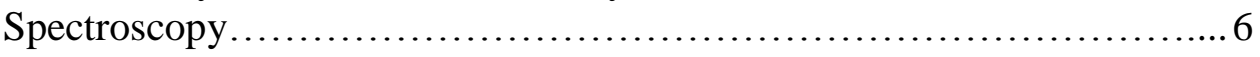

III. Chirped Pulse Millimeter Wave Spectroscopy.......................... 12

Chapter 1. Spectrometer Design and Benchmarking ........................... 18 A segmented chirped pulse Fourier transform spectrometer for high-speed millimeter wave molecular rotational spectroscopy

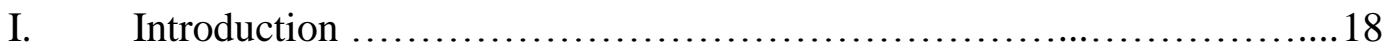

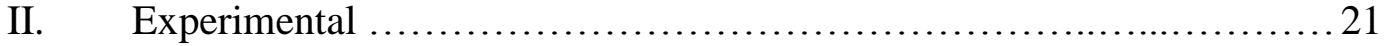

III. Basic Principles of Instrument Design .............................. 24

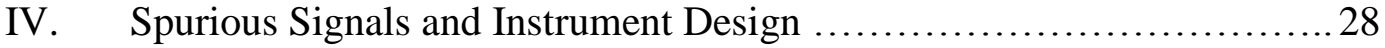

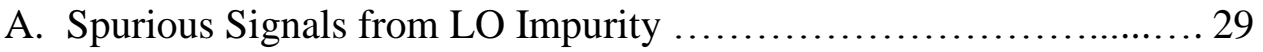

B. Spurious Signals in the Receiver Path from Data Processing .........30

V. Operating Modes of the CP-FTmmW Spectrometer .................... 35

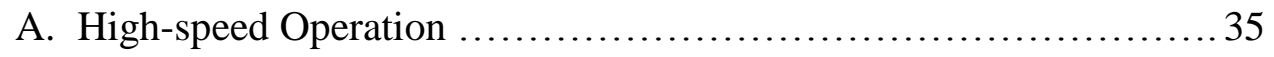

B. High Dynamic Range Operation ................................ 38

VI. Intensity Calibration and Spectral Accuracy .........................41

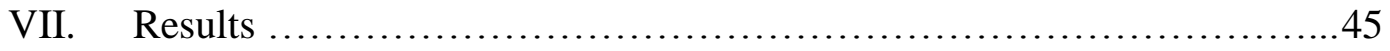

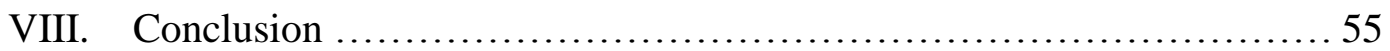

Chapter 2. Analysis Tools ................................................ 65

Advanced gas analysis tools for Fourier transform millimeter spectroscopy

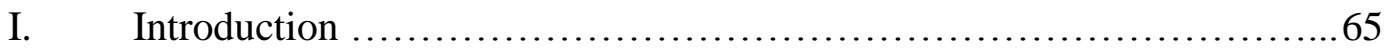

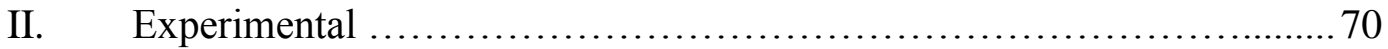


III. Results: Concepts for Advanced Analysis Tools

A. Coherent Excitation ........................................ 74

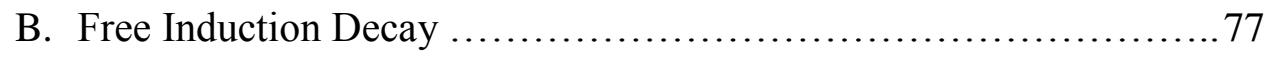

C. Signal Echoes ............................................. 78

D. Mass Estimation ............................................. 79

E. Double Resonance (two color analysis) .......................86

IV. Discussion and Conclusions ................................... 95

Chapter 3. Mixture Analysis-Solutions ....................................103

Direct headspace analysis by chirped pulse Fourier transform millimeter wave spectroscopy

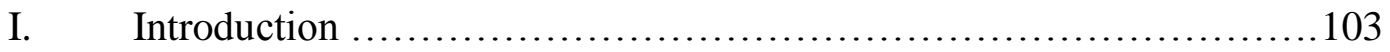

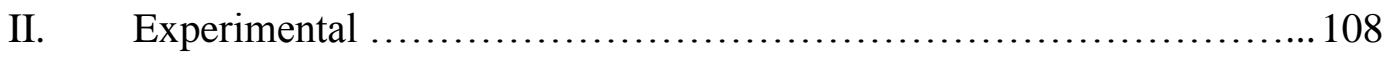

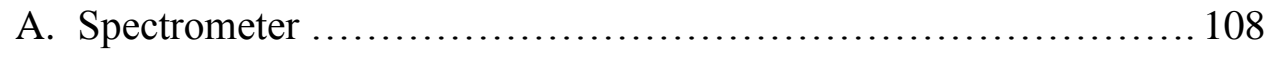

B. Distinctions for CP-FTmmW in Rotational Spectroscopy .......... 110

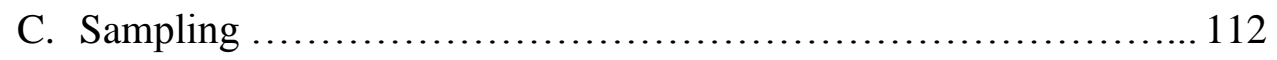

III. Results .................................................. 114

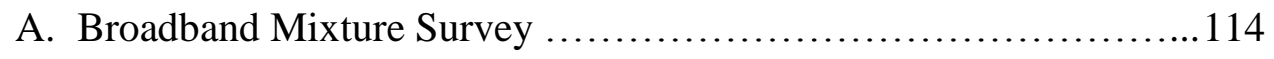

B. Targeted Mixture Survey ................................. 123

IV. Discussion and Conclusions ................................. 129

Chapter 4. Mixture Analysis-Solids .................................... 137

The millimeter wave rotational spectrum of the 4(5)-methylimidazole

tautomeric pair

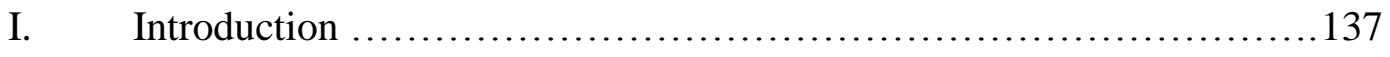

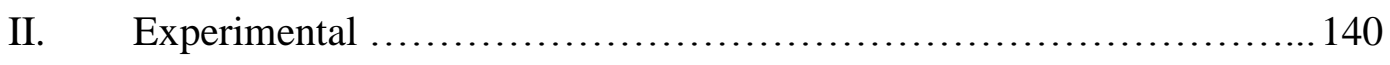

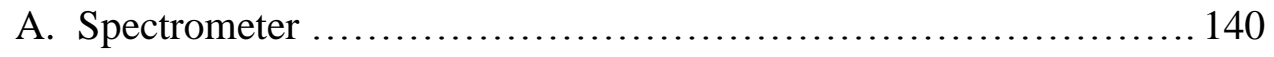

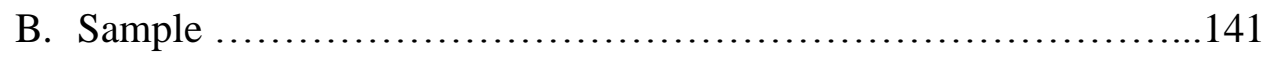


C. Spectral Assignment .................................... 142

III. Results ....................................................... 144

IV. Discussion .................................................. 154

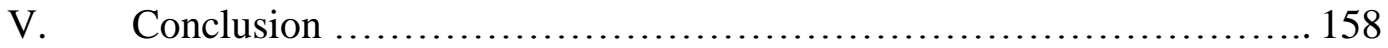

Concluding Chapter: A New Generation for Rotational Spectroscopy .................164 


\section{Acknowledgments and Dedications}

Although a scientist's education never really stops, the $\mathrm{PhD}$ is a major pinnacle on the path to higher knowledge. It is a highpoint with many milestones, each a great challenge on their own. I have had many guides along the way. Scholastic achievement was a value in my family from day one. My aunt Mary Lynne, a $1^{\text {st }}$ grade teacher was always there to answer my early questions and to focus my efforts on presenting my skills. "Even if you do know the material, how can anyone else know if you can't present the answers to them?" I would like to thank my family for all of their support and refocusing through the years. I have been fortunate to have the foresight of my parents and their ambition to create an opportunity at education that I otherwise would not have had. My mother has always been my greatest advocate and one of my greatest examples of how to make the sacrifice to work hard. My father will always be my example of transformation.

I would also like to thank my first research advisor, Professor Paul Sharp for taking me in as an early apprentice in my undergraduate studies and enriching my knowledge of metal chemistry. His support and that experience ultimately led me to the University of Virginia, where I had the opportunity to develop a diverse skillset for scientific discovery. I am truly proud and privileged to have been a part of the research group of Brooks Pate. The opportunity to learn from such a forward thinking, bigpicture, and genuinely innovative mentor has imprinted an approach to problem solving that I believe will be the foundation to my contributions throughout my career. My dissertation committee has also provided invaluable direction: Kevin Lehmann, John Yates, Eric Herbst, and Stephen Macko. I would also like to thank Mark Smith, Oliver 
Monti, and Lucy Ziurys for the crucial foundation of knowledge I developed at the University of Arizona.

The last group of people I would like to thank are those that have been in the trenches with me though out my graduate experience, my lab mates. They have been just as important to my training and growth. My lab mentors: Lindsay Zack, Emmy Tenenbaum, Ming Sun, and Justin Neill. My lab mates: Danny Zaleski, Nathan Seifert, Matt Muckle, Jei Min, Gilles Adande, Jessica Edwards, Matt Bucchino. My lab partner: Amanda Steber. And, finally, my closest lab partner, my closest friend and my wife: Robin Pulliam. 


\section{Introductory Chapter: Astrochemistry}

\section{The origins of millimeter wave analytical chemistry}

Molecular rotational spectroscopy has been a relatively isolated field of study since its introduction around the mid- $20^{\text {th }}$ century. Rotational spectra are used as fundamental tool in physical chemistry for determining the molecular structure of gas phase molecules, and in astrochemistry for investigating the composition of the interstellar medium (ISM). However, there has been a lag in the widespread adoption of rotational spectroscopy for practical analytical chemistry applications. The ambition to broadcast the utility of this method to a diverse set of research scientists has recently driven the development of new techniques that improve performance and practicality for simple, benchtop rotational spectroscopy. It is important to note that, although rotational spectroscopy would be a new tool for analytical chemists, it is a very mature technique for astrochemists. In fact, astrochemistry has been the only major analytical application of rotational spectroscopy and has played a large role in the story leading up to the current age of rotational spectroscopy, paving the way to where it may finally "take its place along with the rest of the electromagnetic spectrum in the tools of an analytical chemist." [1]

\section{Chemistry in the Interstellar Medium}

Astronomical and astrophysical studies date back well before the start of the $20^{\text {th }}$ century. Discoveries of new planets and investigations into the general composition of space have helped to tell the story of how the earth, and its life came to be. However, the 
formation of life may have as much to do with the cycling of bulk matter as with the cycling of molecular material. It wasn't until the mid- $20^{\text {th }}$ century that the scope of space research really expanded to include chemistry. Space research, with regards to chemistry, has been defined in rather broad terms. For example, chemistry in stellar atmospheres refers to the ionized elemental make-up investigated through atomic spectroscopy (where metallicity refers to composition of atoms heavier than helium), [2] and cosmochemistry is a field largely concerned with the make-up of solid refractory materials. [3] The study of chemical reactions and the molecular composition of the ISM is the domain of Astrochemistry.

Early ISM observations in the visible spectrum during the 1930s indicated the presence of the diatomic molecules like $\mathrm{CH}, \mathrm{CN}$, and $\mathrm{CH}^{+}$. [4] From the onset, line overlap complicated spectral assignments and it was recognized that superior resolution and spectral redundancy were key to confident composition analysis. Chemical separation on Earth can help solve the complex mixture analysis problem, but for studying the ISM, astronomers had to develop spectroscopic techniques that were inherently capable of high chemical selectivity. Molecular spectroscopy in the microwave and millimeter wavelength region, where molecular rotational transitions are measured, provided the superior resolution required and became the primary spectral window for establishing the comprehensive chemical inventory of the ISM.

Astrochemistry is considered to have been launched in the 1970s, when radio telescopes were used to detect polyatomic molecules in numerous sources from their pure rotational spectrum (as opposed to ro-vibration in FIR spectroscopy). Detections of water, ammonia, and formaldehyde at microwave frequencies below $40 \mathrm{GHz}$ highlighted 
the prebiotic nature of ISM chemistry. [5-7] The first radio detection, however, was of the $\mathrm{OH}$ free radical and came after Zeeman modulated microwave spectrometers enabled the measurement of highly accurate rest frequencies. [8,9] The cross dependency of advances in laboratory technology and observational astronomy technologies is a strong theme, which is apparent even in the present day. By the late 1970s, the spectral coverage of laboratory techniques and telescope instrumentation had been extended into the millimeter wave region, where the rotational spectrum of a broader set of heavier molecules would be accessible. The National Radio Astronomy Observatory (NRAO) built the Kitt Peak 36 foot millimeter wave telescope, and during early measurements made the first millimeter detection of carbon monoxide in the ISM. The assignment of the spectrum was indisputable and initiated the expansion of chemical knowledge in the ISM to include a very diverse molecular inventory. [10] New molecular detections in space would follow at a rate of about four per year, continuing to the present day.

Many of the molecules detected in the ISM are what chemists would consider to be transient, or unstable species such as radicals, ions, protonated species, and highenergy isomers. Molecules detected in the ISM are largely composed of hydrogen, carbon, oxygen and nitrogen, yielding plenty of stable molecules, as well, many of which are pre-biotic in nature providing synthetic routes to sugars and amino acids. [11,12] In some cases, silicon, sulfur, and phosphorous analogues to the NCO species are also abundant. [13-15] Even simple, metal bearing molecules have been detected; they are generally halogenated (or oxygenated) diatomics, or cyano-triatomics with an s-, p-, or d-block metal. [16-25] The more complex molecules (> 10 atoms) detected in the ISM 
also include unsaturated, conjugated carbon chains. [26] Together, this combined chemical inventory in space represents a very diverse group of molecular species.

New molecule detections are historically focused on one of a few chemical "factories" in the ISM: 1) molecular clouds: e.g. Sagittarius B2N, Orion KL, and the Taurus Molecular Cloud (TMC-1), and 2) circumstellar envelopes of evolved stars: e.g. IRC+10216 and VY-Canis Majoris. Molecular line surveys can be found for all of these objects. [27-31] Common to each source is a material for seeding chemistry, such as ice or a central star, and a dense environment for shielding against destructive UV rays. These particular clouds and circumstellar envelopes are chosen for chemical searches because of their proximity to earth, their size, and the historically established high abundance of molecular material. For environments with very active chemistry, the gas density can range from $10^{3}-10^{8} \mathrm{~cm}^{-3}$ and kinetic temperatures from $10 \mathrm{~K}-300 \mathrm{~K}$. Though they provide for sensitive spectroscopy, the five sources are by no means the only examples of chemically rich environments. Dark clouds, diffuse clouds, circumstellar envelopes, comet tails and coma, and planetary nebulae throughout the ISM have been the focus of numerous chemical studies, which provide insight into chemical processes via molecular rotational spectroscopy.

Since it is not currently feasible to collect a gas sample of interstellar material for chemical analysis, the spectroscopy must be sufficiently selective to determine the individual components of astrophysical environments. The high-resolution rotational spectrum is absolutely specific to a particular molecular structure and is one of the most stringent identifiers of a molecule. Any change in mass distribution, even as slight as an isotopic substitution, changes the moment of inertia enough to substantially shift the 
rotational spectrum. The second feature that enables the superior selectivity of the rotational spectrum is the inherently high-resolution $\left(\Delta v / v \approx 10^{-5}\right.$ or $10^{-4}$ for expanding gases in the ISM) due to the comparatively long lived excited rotational states and the low Doppler limit at millimeter wavelengths. Even in complex mixtures, it is possible to analyze the composition with confidence based on a rotational spectrum as evidenced by the fact that most of the molecular detections in the ISM are common to the same sources. After considering source velocity with respect to the local standard of rest (LSR, the common reference frame for reporting astrophysical velocities in order to account for Doppler shift), the center line frequencies of a molecular millimeter rotational spectrum can be determined to an accuracy of better than $1 \mathrm{ppm}$ in total agreement across most sources in the ISM and with the laboratory rest frequencies.

In some cases, the ISM has been the first indicator of a new molecule, such as the detection of $\mathrm{HCO}^{+}$in the 1970 s, which had never before been measured by rotational spectroscopy. [32] However, in general, laboratory measurements are needed as a reference for astronomical observations when determining molecular composition. The two measurements are used in concert to catalogue the chemistry of interstellar space, but there is an important and obvious distinction. In a terrestrial environment, scientists can control the measurement by trying to mimic interstellar conditions, i.e. working in a vacuum and providing an appropriate source of energy (heat, electric discharge, laser, etc.); however, they cannot sufficiently reproduce the actual environment of the ISM. Molecules that are abundant in the ISM, for example radicals and ions, may not be abundant in the laboratory. In fact, some molecules may not even have been considered in the laboratory as viable candidates for astronomical searches. Thus, laboratory 
scientists are careful to archive data, so it can be re-examined as new astronomical observations (or conclusions) become available. The converse is also true for broadband astronomical survey data. $[11,33,34]$

In general, conditions in the ISM induce a very exotic chemistry that provides a challenge for laboratory synthesis. In all sources, cosmic rays penetrate into the dense material and influence the molecular composition by ionization and inducing photochemistry on the diffuse edges. [35,36] A background radiation field can be the dominant source of energy in a non-uniform environment. Generally, thermodynamic equilibrium is not a requirement for the ISM where collisions in a molecular cloud can occur on the time scale of hours to years. As a result, the radiation field and the thermodynamic temperature both provide energy for chemical reactions and spectroscopic energy transitions. Many observations do not indicate thermodynamic equilibrium; for example, $\mathrm{SiO}$, water, and methanol masers are the extreme case where transitions with very high excitation temperatures $(>1000 \mathrm{~K})$ are observed in a molecular spectrum. These physical conditions in the ISM are difficult to emulate in terrestrial experiments; however, laboratory techniques have so far enabled the assignment of nearly $50-80 \%$ of the features in astronomical line surveys. New astronomical instruments, with greatly improved sensitivity and broadband capabilities, will further drive the need for improved laboratory techniques.

\section{Chemical Synthesis and Detection by Molecular Rotational Spectroscopy}

Pure rotational transitions occur from centimeter to submillimeter wavelengths, between the radio and infrared regions of the electromagnetic spectrum. There is some 
overlap and ambiguity in the terms used to specify this region. In present day spectroscopy literature, microwave (or "centimeter wave"), millimeter wave, and submillimeter wave commonly refer to frequencies lower than approximately $50 \mathrm{GHz}$, between 50 and $300 \mathrm{GHz}$, and from $300 \mathrm{GHz}$ up to $1 \mathrm{THz}$, respectively. Molecules exhibit pure rotational transitions throughout this wide spectral space. Considering the high-resolution nature of rotational spectroscopy where typical line widths are a factor of 1000 to 10000 times narrower than in IR spectroscopy, for example, this is a very wide window.

Through the 1960s and 1970s, there was little fundamental distinction between microwave and millimeter laboratory experiments. However, microwave and millimeter/submillimeter spectroscopic techniques diverged at the beginning of the 1980s with the invention of the supersonic jet and Fourier transform microwave (FTMW) cavity emission spectroscopy. [37,38] Static gas FTMW cavity emission spectroscopy was also described at this time. [39] In recent years, the distinction between microwave and millimeter/submillimeter has become clear because laboratory measurements in the two spectral regions are now carried out with a different fundamental basis in technology and technique. After the introduction of FTMW, continuous flow absorption cells would continue on a development path almost exclusively for millimeter/submillimeter spectroscopy.

Microwave studies are usually carried out under cold conditions (1-50 K), where the lowest energy levels of molecules are populated. The molecules are cooled via adiabatic expansion of a gas into a vacuum, the so-called supersonic jet or molecular beam. The benefits of using pulsed molecular beam FTMW methods include thermal 
cooling for observing weakly bound molecules and complexes, increased single acquisition column density over constant pressure conditions, and an effective increase in optical path length by cavity enhancement. Furthermore, since FTMW is a time domain technique, the molecular emission is detected independently from the pulsed excitation light yielding the advantage of detecting clean molecular signals against zero background light. The signal generator and cavity for FTMW spectrometers can be tuned across a sufficiently wide bandwidth to measure a broad range of molecular transitions and achieve excellent resolution for highly accurate molecular structure determination. However, these data are often inadequate to calculate the higher transitions within the desired $0.1 \mathrm{ppm}$ accuracy in the submillimeter spectrum. At the higher angular momentum states measured by submillimeter spectroscopy, centrifugal distortion becomes a significant perturbation to the rigid rotor spectrum; for free-radicals, electron angular momentum is an additional perturbation.

Millimeter/submillimeter spectra are collected by direct absorption in a low pressure ( $<100$ mTorr), continuous flow cell under warm conditions ( > $200 \mathrm{~K}$ ) that may be held at room temperature or heated. This spectral region has been pursued for decades by astrochemists where warm organic molecules $(<100 \mathrm{amu})$ and some smaller inorganic molecules have their most intense transitions. Historically, methods for generating, propagating, and detecting radiation at short submillimeter wavelengths have posed challenges that have delayed the introduction of Fourier transform methods used at longer wavelengths. One target for sensitivity improvement was path length extension accomplished through double pass optics, which eventually became standard for millimeter/submillimeter spectrometers. [40] However, a factor of two improvement did 
not compare to the sensitivity gains of FTMW. Power output, frequency accuracy, and frequency stability all become a greater challenge at higher frequencies. Nonetheless, a global measurement across as much of the rotational spectrum as possible is crucial for analyzing complex mixtures at the wide range of temperatures found in the ISM.

\section{Synthetic methods:}

Although the peak absorption (or emission) of a molecular rotational spectrum depends on temperature, under laboratory conditions this parameter is typically chosen to create the best conditions for chemical production. The exotic chemistry of the ISM has driven new approaches to producing gas phase molecules in laboratory environments. Thus, laboratory astrochemistry is, perhaps, as much chemical synthesis as it is spectroscopy. Since the turn of the $20^{\text {th }}$ century, scientists have developed techniques for synthesizing short-lived molecules such as free-radicals, electronically excited molecules, high-energy conformers, ions, and van der Waals complexes with composition across the periodic table. A common scheme involves applying an external source of energy in the form of an electrical discharge, a laser, or heat to a sample in a molecular beam or some kind of gas flow. These methods have been modified over the years with the goal of improving chemical production.

A typical energy source for producing transient molecules in the laboratory is the electrical discharge, introduced in Zeeman modulated spectrometers for producing free radicals. [41,42] Both the discharge and the sample cell are direct influences to optimum synthesis. The Zeeman modulated spectrometer cell was a modification of the first Stark modulated spectrometer, [43,44] comprised of a main chamber for the microwave 
propagation and an additional section attached below that was outfitted with two opposing electrodes for producing radicals by supplying either dc or oscillating current in the presence of 100 mTorr of an inert gas to sustain a "glow" (e.g. Argon). Lower pressure, Doppler limited glows could be sustained once the glow cell was incorporated into the larger volume, measurement cell. [45]

From early implementations, chemical synthesis was an optimization process. Good production conditions are a complicated function of discharge conditions (current, voltage, etc.), temperature, and time, as reaction products coat and/or corrode the cell and electrodes over the course of minutes or hours, ultimately reducing the discharge current. In addition, the choice of chemical reactants and inert discharge carrier gases will influence production. [46] In many experiments, radical production can be improved by cooling the cell with a nitrogen jacket. [47] Experimental results in glow discharge cells are difficult to reproduce but some general trends in the reagents, discharge conditions, and pressures have been noted.

The electrodes can provide a dual function: producing the glow for synthesis and verifying the presence of an ion. Ions in the discharge travel with a velocity shift depending on the charge/mass ratio and the polarity of the discharge. Spectral lines arising from an ionic carrier can be velocity shifted in the propagation path by reversing the discharge polarity. [48] The ion property of velocity shift has been exploited for the design of velocity modulated submillimeter spectrometers. [49]

Free radical synthesis methods based on electric discharge have been used to characterize many of the molecules of the ISM; however, successful synthesis of metal bearing molecules for submillimeter measurements has traditionally required the 
introduction of metal vapor. [50,51] Metal bearing chemicals can be purchased, but much success has been met by vaporizing metals for reaction. Vaporization can be accomplished by oven heating, [52,53] heating by contact with a discharge electrode, [54] or by sputtering of the electrode itself. [55] The heat, in addition to the discharge, can provide the energy necessary to excite metals into higher electronic states, from which they may react more readily.

In recent years, pulsed techniques in microwave spectroscopy have combined the benefits of FTMW spectroscopy with transient sources of energy for synthesis such as lasers. Laser ablation of metals, [56] sometimes in combination with an electrical discharge, [57] has been the experimental technique for numerous microwave structure studies of metal bearing molecules produced in molecular beams by supersonic jet expansion. Radicals are short-lived after a laser pulse, so the discharge can be used to stabilize them for measurement. [58] In addition to metal rods, laser ablation can be used to vaporize solid organic species from compressed powders. [59]

Lasers can also be used to induce photochemistry. Attempts at flash photolysis date back to the 1950s, [42] with these techniques eventually applied to gas phase kinetic measurements. [60] Early implementations used a mercury lamp for a flash discharge across a sample in mercury vapor. Later studies utilized an excimer laser to generate radicals. [61] Conventional electric discharge spectroscopy can be used to generate electronically excited molecules, however laser techniques were eventually developed for studying electronically excited molecules by excimer laser photolysis. [62,63]

Pulsed lasers are compatible with the pulsed molecular beam used in microwave spectroscopy. Millimeter wave jet absorption spectrometers have also been in operation 
for a few years. $[64,65]$ In addition to pulsed discharge, laser ablation has been used in combination with millimeter-wave jet absorption spectroscopy. [66] These millimeter/submillimeter molecular beam spectrometers keep the pulsed synthesis capabilities and retain the path length advantage in a slightly different way than their FTMW counterparts, which use a Fabry-Perot high Q cavity. In the Supersonic Jet

Spectrometer for Terahertz Applications (SuJeSTA), the "cavity" is essentially a multipass beam path accomplished with curved mirrors. In the OROTRON spectrometer, an intracavity molecular beam is created inside the resonant cavity of the millimeterwave generator. SuJeSTA, OROTRON, and modern spectrometers for fast direct absorption spectroscopy all underscore the continued ambition of scientists to leverage the benefits of high frequency spectroscopy for discovery.

\section{Chirped Pulse Millimeter Wave Spectroscopy}

Pure rotational spectroscopy has passed many milestones across the decades, most of which have improved synthetic techniques. But, with the exception of FTMW, there had been few advances in the actual methods for measuring these spectra at microwave and millimeter/submillimeter wavelengths until the introduction of chirped pulse (CP) FTMW in 2006. [67, 68] The previous generation, narrow band cavity FTMW spectrometers had brought a significant advance and a fresh technique, but they did not fully realize the multiplexing advantage that usually comes with Fourier transform spectroscopy. CP-FTMW instruments turned microwave spectroscopy into a broadband method and removed the traditional trade off between FTMW spectroscopy and millimeter/submillimeter direct absorption spectroscopy. By any method, it is a 
challenge to control gas phase chemical synthesis of transient molecules. At present, structural studies of reactive molecules using millimeter/submillimeter spectroscopy are still conducted by continuous flow, static gas, and molecular beam methods where fluctuations in production may introduce inconsistencies in the data over the time required to scan a sufficiently wide spectral region for analysis. As such, broadband acquisition speed can be considered one of the next tools for sensitive millimeter/submillimeter spectroscopy that favors room temperature rotational spectra.

New millimeter/submillimeter telescope arrays have been designed with the broadband advantage in mind and have fueled some significant advances in high frequency, solid-state light sources which have been incorporated into modern millimeter/submillimeter spectrometers. The latest direct absorption spectrometers accomplish sensitive, full spectral sweeps ( > $10 \mathrm{GHz}$ ) in seconds. [69-71] However, chirped pulse spectroscopy, can deliver broadband sensitivity gains of $>100$ fold over absorption spectrometers. In the early days of development, the goal was to design a chirped pulse spectrometer that could match the data throughput of new millimeter/submillimeter telescope arrays. In addition to meeting the needs of the astronomy community, the millimeter wave chirped-pulse spectrometer that has resulted from this work is a strong design for practical, high performance analytical chemistry instruments. Though the history of spectroscopy at millimeter/submillimeter wavelengths may have been driven by astrochemistry, the advent of this new technology gives its future a much broader scope. In this thesis, the development of a new, segmented chirped pulse millimeter wave spectrometer is presented, with a demonstration of its strengths as an analytical tool applicable to many fields of science. 


\section{References for Introductory Chapter:}

1. I. R. Medvedev, M. Behnke, and F. C. De Lucia, Analyst, 2006, 131, 1299.

2. D. F. Gray, Chemical Analysis. In The Observation and Analysis of Stellar Atmospheres; Cambridge, New York, 1992, Cambridge University Press, p. 302.

3. G. R. McSween, H. Y. Huss, Cosmochemistry, New York, 2010, Cambridge University Press.

4. A. McKellar, Pub. ASP, 1940, 52, 187.

5. A. C. Chueng, D. M. Rank, C. H. Townes, D. D. Thornton, W. J. Welch, Nature, 1969, 221, 626.

6. A. C. Chueng, D. M. Rank, C. H. Townes, D. D. Thornton, W. J. Welch, Phys. Rev. Lett., 1968, 21, 1701.

7. L. E. Snyder, D. Buhl, B. Zuckerman, P. Palmer, Phys. Rev. Lett., 1969, 22, 679.

8. S. Weinreb, A. H. Barret, M. L. Meeks, J. C. Henry, Nature, 1963, 200, 829.

9. G. Ehrenstein, C. H. Townes, M.J. Stevenson, Phys. Rev. Lett., 1959, 3, 40.

10. R. W. Wilson, K. B. Jefferts, A. A. Penzias, ApJ, 1970, 161, L43.

11. R. A. Loomis, D.P. Zaleski, A.L. Steber, J.L. Neill, M.T. Muckle, B.J. Harris, J.M. Hollis, P.R. Jewell, V. Lattanzi, F.J. Lovas, O. Martinez, M.C. McCarthy, A.J. Remijan, B.H. Pate, and J.F. Corby, ApJ, 2013, 765, L9.

12. J. M. Hollis, P. R. Jewell, F. J Lovas, A. Remijan, H. Mollendal, ApJ, 2004, 610, L21.

13. D. M. Goldhaber, A. L. Betz, ApJ, 1984, 279, L55.

14. M. W. Sinclair, N. Fourikis, J. C. Ribes, B. J. Robinson, R. D. Brown, P. D. Godfrey, Aust. J. Phys., 1973, 26, 85.

15. M. Agundez, J. Cernicharo, J. R. Pardo, M. Guelin, T. G. Phillips, $A \& A$, 2008, 485, L33.

16. L. N. Zack, D. T. Halfen, L. M. Ziurys, ApJ, 2011, 733, L36.

17. J. Cernicharo, M. Guelin, A\&A, 1987, 183, L10. 
18. T. Kaminski, C. A. Gottleib, K. M. Menten, N. A. Patel, K. H. Young, S. Brunken, H. S. P. Muller, M. C. McCarthy, J. M Winters, L. Decin, A\&A, 2013, 551, A113.

19. E. D. Tenenbaum, L. M. Ziurys, ApJ, 2009, 694, L59.

20. L. M. Ziurys, C. Savage, J. L. Highberger, A. J. Apponi, M. Guelin, J. Cernicharo, ApJ, 2002, 564, L45.

23. K. Kawaguchi, E. Kagi, T. Harano, S. Takano, S. Saito, ApJ, 1993, 406, L39.

24. B. E. Turner, T. C. Steimle, L. Meerts, ApJ, 1994, 426, L97.

25. L. M. Ziurys, A. J. Apponi, M. Guelin, J. Cernicharo, ApJ, 1995, 445, L47.

26. M. B. Bell, P. A. Feldman, M. J. Travers, M. C. McCarthy, C. A. Gottlieb, P. Thaddeus, ApJ, 1997, 483, L61.

27. D. N. Friedel, L. E. Snyder, B. E. Turner, A. Remijan, ApJ, 2004, 600, 234.

28. A. O. H. Olofsson, C. M. Persson, N. Koning, P. Bergman, P. F. Bernath, J. H. Black, U. Frisk, W. Geppert, T. I. Hasegawa, A. Hjalmarson, A\&A,2007, 476, 791.

29. N. Kaifu, M. Ohishi, K. Kawaguchi, S. Saito, S. Yamamoto, T. Miyaji, K. Miyasawa, S. Ishikawa, C. Noumaru, S. Harasawa, PASJ, 2004, 56, 69.

30. N. A. Patel, K. Y.Young, C. A. Gottleib, P. Thaddeus, R. W. Wilson, K. M. Menten, M. J. Reid, M. C. McCarthy, J. Cernicharo, J. He, et al., ApJ, 2011, 193, 17.

31. E. D. Tenenbaum, J. L. Dodd, S. N. Milam, N. J. Woolf, L.M. Ziurys, ApJ, 2010, 190, 348.

32. D. Buhl, L.E. Snyder, Nature, 1970, 228, 267.

33. J. L. Neill, M. T. Muckle, D. P. Zaleski, A. L. Steber, B. H. Pate, V. Lattanzi, S. Spezzano, M. C. McCarthy, A. J. Remijan, ApJ, 2012, 755, 153.

34. D. P. Zaleski, N. A. Seifert, A.L. Steber, M.T. Muckle, R. A. Loomis, J.F. Corby, O. Martinez, K.N. Crabtree, P.R. Jewell, J.M. Hollis, F.J. Lovas, D. Vasquez, J. Nyiramahirwe, N. Sciortino, K. Johnson, M.C. McCarthy, A.J. Remijan, B.H. Pate, ApJ, 2013, 765, L10.

35. D. Ruffle, E. Herbst, MNRAS, 2001, 332, 770.

36. R. T. Garrod, E. Herbst, $A \& A, 2006,457,927$. 
37. E. J. Campbell, L. W. Buxton, T. J. Balle, M. R. Keenan, W. H. Flygare, J. Chem. Phys., 1981, 74, 829.

39. W. H. Balle, T. J., Flygare, Rev. Sci. Instrum., 1981, 53, 33.

39. E. J. Campbell, J. Chem. Phys., 1981, 74, 813.

40. H. E. Radford, W. Wei, T. J. Sears, J. Chem. Phys., 1992, 97, 3989.

41. G. C. Dousmanis, T. M. Sanders, C. H. Townes, Phys. Rev., 1955, 100, 1735.

42. I. R. Hurle, T. M. Sugden, Jounal Sci. Instruments., 1958, 35, 319.

43. R. H. Hughes, E. B. Wilson, Phys. Rev., 1947, 71, 562.

44. A. H. Sharbaugh, Rev. Sci. Instrum., 1959, 21, 120.

45. R. C. Woods, Rev. Sci. Instrum., 1973, 44, 282.

46. F. Kaufman, F. P. Del Greco, J. Chem. Phys., 1961, 34, 61.

47. T. A. Dixon, R. C. Woods, Phys. Rev. Lett., 1975, 34, 13.

48. R. C. Woods, T. A. Dixon, Phys. Rev. Lett., 1975, 35, 1269.

49. C. Savage L. M. Ziurys, Rev. Sci. Instrum., 2005, 76, 043106.

50. M. L. Stitch, A. Honig, C. H. Townes, Rev. Sci. Instrum., 1954, 25, 759.

51. P. A. Tate, M. W. Strandberg, Rev. Sci. Instrum., 1954, 25, 956.

52. K. Kawaguchi, E. Kagi, T. Hirano, S. Takano, S. Saito, ApJ, 1993, 406, L39.

53. L. M. Ziurys, W. L. Barclay, M. A. Anderson, D. A. Fletcher, J. W. Lamb, Rev. Sci. Instrum., 1994, 65, 1517.

54. T. Okabayashi, M. Tanimoto, ApJ, 2000, 543, 275.

55. M. Yamazaki, E., Okabayashi, T., Tanimoto, ApJ, 2001, 551, L199.

56. R. D Suenram, F. J. Lovas, G. T. Fraser, K. Matsumara, J. Chem. Phys., 1990, 92 , 4724.

57. M. Sun, D. T. Halfen, J. Min, B. Harris, D. J. Cloutheir, L. M. Ziurys, J. Chem. Phys., 2010, 133, 174301. 
58. J. S. Lash, R. M. Gilgenback, C. H. Ching, Appl. Phys. Lett., 1944, 65, 53.

59. A. Lesarri, S. Mata, J. C. Lopez, J. L. Alonso, Rev. Sci. Instrum., 2003, 74, 4799.

60. Y. Endo, H. Kanamori, E. Hirota, Laser Chem., 1987, 7, 61.

61. W. F. Kolbe, B. Leskovar, Rev. Sci. Instrum., 1985 56, 1577.

62. S. Saito, J. Chem. Phys., 1970, 53, 2544.

63. M. Bogey, S. Civis, B. Delcroix, C. Demuynck, A. F. Krupnov, J. Quiguer, M. Y. Tretyakov, A. Walters, J. Mol. Spec., 1997, 97, 85.

64. M. Caris, T. F. Giesen, C. Duan, H. S. P. Müller, S. Schlemmer, K. M. T. Yamada, J. Mol. Spec., 2009, 253, 99.

65. L. A. Surin, B. S. Dumesh, F. Lewen, D. A. Roth, V. P. Kostromin, F. S. Rusin, G. Winnewisser, I. Pak, Rev. Sci. Instrum., 2001, 72, 2535.

66. P. Kania, M. Hermanns, S. Brunken, H. S. P. Muller, T. F. Giesen, J. Mol. Spec., 2011, 268, 173.

67. G. G. Brown, B. C. Dian, K. O. Douglass, S. M. Geyer, B. H. Pate, J. Mol. Spec., 2006, 238, 200.

68. G. G. Brown, B. C. Dian, K. O. Douglass, S. M. Geyer, S. T. Shipman, B. H. Pate, Rev. Sci. Instrum., 2008, 79, 053103.

69. I. R. Medvedev, C. F. Neese, G. M. Plummer, F. C. De Lucia, Opt. Lett., 2010, 35, 1533.

70. R. A. Motiyenko, L. Margulès, E. A. Alekseev, J. C. Guillemin, J. Demaison, J. Mol. Spec., 2010, 264, 94.

71. B. J. Drouin, F. W. Maiwald, J. C. Pearson, Rev. Sci. Instrum., 2005, 76, 093113. 


\title{
Chapter 1. Spectrometer Design and Benchmarking
}

\author{
A segmented chirped pulse Fourier transform spectrometer for high-speed \\ millimeter wave molecular rotational spectroscopy
}

\section{Introduction}

Rotational spectroscopy was one of the first fields of high resolution molecular spectroscopy and has a strong theoretical foundation for spectrum analysis. [1,2] Molecular rotational spectroscopy involves transitions between the rotational kinetic energy levels of the freely rotating molecule in a low pressure gas that are discrete through the quantization of angular momentum. The rotational spectrum covers all frequencies (limited by molecular dissociation) and the frequency where the peak intensity occurs depends on the molecular size and the temperature of the sample. For molecules and clusters studied in molecular beams, where rotational temperatures of $1-10 \mathrm{~K}$ can be easily achieved, the peak spectral intensity typically falls in the $2-40 \mathrm{GHz}$ frequency range leading to the common terminology of "microwave spectroscopy" for rotational spectroscopy. For gases that can be studied near room temperature, the peak intensity of the rotational spectrum typically falls in the $100 \mathrm{GHz}-1 \mathrm{THz}$ frequency range, using mm-wave, sub mm-wave, and terahertz technology. A spectrometer for mm-wave rotational spectroscopy $(260-295 \mathrm{GHz})$ is described here that extends chirped pulse Fourier transform (CP-FT) methods that have recently been developed for broadband microwave spectroscopy. [3,4]

Most applications of molecular rotational spectroscopy are based on the connection between the rotational spectrum and the three dimensional structure of the 
molecule through the principal moments-of-inertia (which appear in the Hamiltonian as the three rotational constants $(\mathrm{A}, \mathrm{B}$, and $\mathrm{C})$ and are inversely proportional to the principal moments-of-inertia). [5,6] Quantitative structures of molecules and clusters can be determined by measuring the rotational spectrum of singly substituted isotopologues making it possible to generate a three dimensional molecular structure directly from experiment. [7] The close connection between the rotational spectrum and the molecular geometry, coupled with the availability of spectrometers that produce highly resolved "line spectra" offers benefits for analytical chemistry applications where high selectivity is required. Because the rotational spectra of molecules with isotopes (like ${ }^{13} \mathrm{C}$ ) located at different atom positions are distinct, the technique has advantages over mass spectrometry techniques for identifying the site of isotopic enhancement. This sitespecific isotopologue abundance information often carries important information about the chemical formation routes through secondary kinetic isotope effects. An important recent advance in molecular rotational spectroscopy has shown that the absolute stereochemistry is available through measurements of the phase of the emitted light following coherent excitation of the molecule in a static electric field. [8] This new phenomenon provides a way to obtain the last piece of information about three dimensional structure from experimental study by rotational spectroscopy.

Rotational spectroscopy also has applications in chemical dynamics. Lasermicrowave double-resonance spectroscopy is an important tool for simplifying complex spectra observed in excited vibrational and electronic excited states of molecules. [9-12] The technique is also well suited to detecting reaction products from dissociation reactions where the vibrational dependence of the rotational constants can be used to 
monitor the degree of vibrational excitation. [13-15] This information provides insight into the dynamics at the transition state of the reaction process. Rotational spectroscopy can also be used to study the kinetics of isomerization reactions through motional narrowing and coalescence effects on the overall line shape of the rotational spectrum that are similar to those in nuclear magnetic resonance (NMR) spectroscopy. [16-21] Techniques in mm-wave Fourier transform spectroscopy have also been used to probe the properties of Rydberg states of molecules. [22]

Recent advances in high-speed digital electronics have made it possible to develop broadband techniques for microwave spectroscopy. These new methods use chirped pulse excitation to polarize the gas sample and the spectrum is obtained by digitizing the subsequent broadband molecular emission, or free induction decay (FID). The frequency-domain molecular rotational spectrum is produced by fast Fourier transform of the broadband FID. Several experimental implementations of this technique for spectroscopy at microwave frequencies $(2-40 \mathrm{GHz})$ in pulsed jet expansions have been reported since the technique was introduced in 2006. [3,4, 23-27] For microwave spectroscopy, the sensitivity of the broadband measurement is aided by the availability of high power traveling wave tube and solid-state amplifiers. However, the CP-FT approach has also been shown to work at mm-wave and submillimeter frequencies where only low power light sources are available. [28-31] The instrument design described in this work focuses on developing a CP-FT mm-wave spectroscopy technique with near 100\% measurement duty cycle. The method provides high-speed spectrum acquisition, requiring just $100 \mu \mathrm{s}$ to obtain the spectrum over the full $260-295 \mathrm{GHz}$ spectrometer operating range. High-speed spectrum acquisition leads to relatively poor spurious signal 
performance. The CP-FT spectrometer can be operated in a high dynamic range mode that offers significant spurious signal reduction with a trade off in measurement speed.

\section{Experimental}

A schematic diagram of the spectrometer is shown in Fig. 1.1. The mm-wave light source $(260-295 \mathrm{GHz})$ is an active multiplier chain (AMC) with $24 \mathrm{x}$ frequency multiplication (VDI AMC 291). The source produces $20-45 \mathrm{~mW}$ of power across the 260-295 GHz frequency range. The receiver uses a subharmonic mixer (VDI MixAMC 156) to downconvert the detected mm-wave molecular emission to microwave frequencies. This mixer has a single side band conversion loss of $9 \mathrm{~dB}$ and the IF port bandwidth is $40 \mathrm{GHz}$. Both mm-wave light sources have an input frequency range of $10.7-12.3 \mathrm{GHz}$ and require $15-18 \mathrm{dBm}$ drive power.

The excitation pulses for the source and the local oscillator (LO) for the receiver are generated using an 8-bit arbitrary waveform generator (AWG) operating at $12 \mathrm{GS} / \mathrm{s}$ (Agilent M8190a). The waveforms for chirped pulse excitation and the single frequency LO cover the $1.9-3.4 \mathrm{GHz}$ frequency range. These AWG waveforms are upconverted to the input frequency range of the mm-wave multiplier chains by the circuit shown in Fig. 1.1. This circuit uses a single microwave frequency source, an $8.8 \mathrm{GHz}$ phaselocked dielectric resonator oscillator (PDRO, Microwave Dynamics PLO-4000-8.8GHz) as the LO for two double balanced mixers - one for the excitation pulse channel, and one for the LO channel. A $10 \mathrm{MHz}$ reference is used for input to the PDRO and is provided 


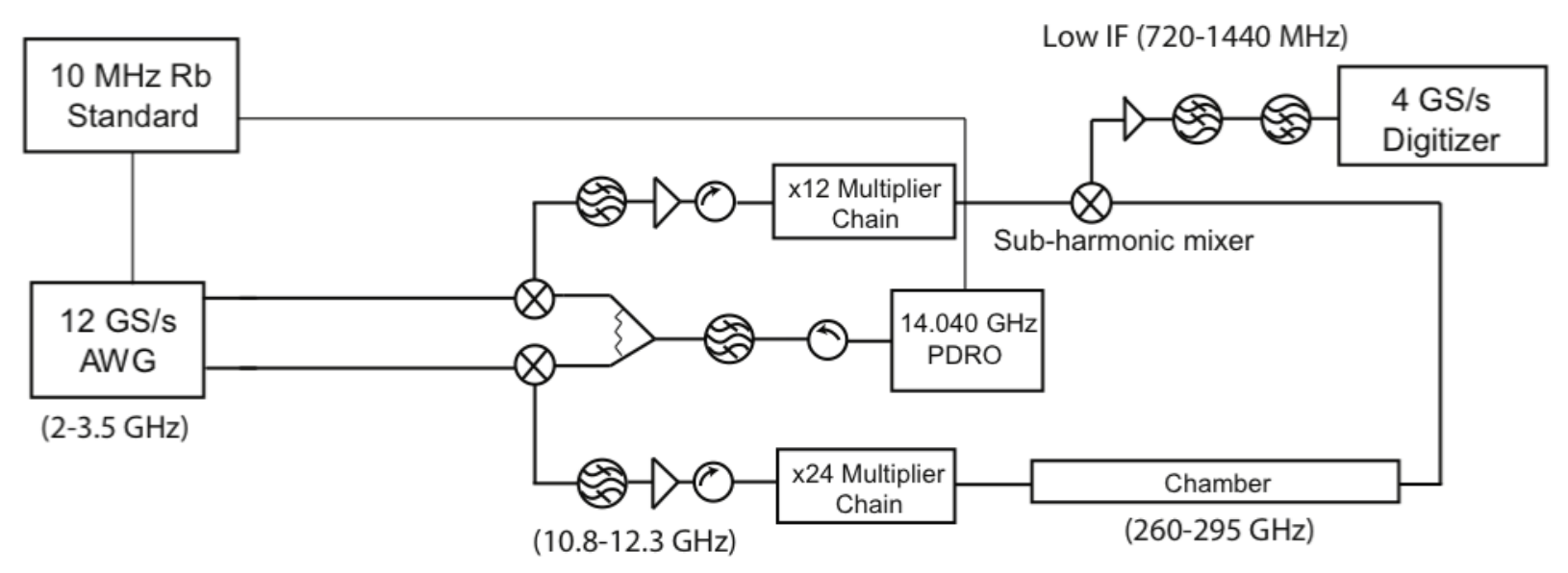

Figure 1.1: A block schematic of the CP-FTmmW spectrometer. A dual channel AWG generates the excitation pulse and the LO waveform. The AWG waveforms are upconverted using the PDRO and multiplied in the AMCs. Molecular emission is downconverted in the SHM and the IF amplification is accomplished with cascaded low band general purpose LNAs separated by a switch in order to protect the final amplification stage from excitation pulse saturation. The resulting low frequency IF is digitized on a PCI digitizer. 
by a rubidium frequency standard (SRS FS725). The AWG also uses this $10 \mathrm{MHz}$ reference clock. The upper sideband from each mixer is selected using a multi-stage cavity bandpass filter (Lorch 13EZ5-11450/A 1700-S) and amplified (Wright Technologies ASG-183020) to reach the required drive power of the AMCs.

The receiver consists of the 12 AMC driven subharmonic mixer (SHM), two cascaded low noise IF amplifiers separated by a switch to protect the final amplification stage from the excitation pulse, and a bandpass filter $(600-1700 \mathrm{MHz}$ constructed using a separate high pass and low pass filter). The IF amplification components (by Mini Circuits) are as follows: ZX60-P162LN+ (P1-dB: 15 dBm Gain: $20 \mathrm{~dB}$ Noise Figure: 0.4 dB), ZYSWA-2-50DR (6ns rise/fall switch), ZX60-H242+ (P1-dB: $15 \mathrm{dBm}$ Gain: $15 \mathrm{~dB}$ Noise Figure: $3.0 \mathrm{~dB}$ ). The downconverted signals in the $720-1440 \mathrm{MHz}$ frequency band are digitized using an 8-bit, 4 GS/s digitizer (Agilent Acqiris U1084a). This digitizer has an on-board 32-bit field programmable gated array (FPGA) that allows signal accumulation up to $2^{24}$ spectra (about 16 million) with a maximum record length of $512 \mathrm{kpts}$ for real-time signal averaging. Following signal acquisition in the time domain, the spectral data is transferred to the host computer for data processing including fast Fourier transformation to produce the frequency-domain rotational spectrum for analysis.

The sample cell is a $65 \mathrm{~cm}$ long, $3.8 \mathrm{~cm}$ diameter stainless steel tube (approximately $1 \mathrm{~L}$ of volume). The input and output windows of the tube are $30 \mathrm{~cm}$ focus, Teflon lenses (Thor Labs LAT300). The light from the mm-wave source is collimated using an off axis parabolic mirror and makes a single pass through the sample cell with an approximately $2 \mathrm{~cm} 3 \mathrm{~dB}$ beam waist. The light exiting the cell is focused into the horn antenna of the mm-wave mixer using a second off-axis parabolic mirror. 
The sample cell is attached to a small turbo pump backed with a dry scroll pump (Agilent 96982147) and the cell pressure is monitored with a MKS Baratron Capacitance Manometer (range 50 mTorr) pressure gauge. The cell includes a vacuum port and a port to introduce chemical gas samples. All chemical samples used in this work were obtained from Aldrich Chemical and used without further purification.

\section{Basic Principles of Instrument Design}

The spectrometer uses chirped pulses (with linear frequency sweep) to polarize the gas sample. The advantage of a chirped excitation pulse compared to a singlefrequency, transform limited pulse is that the waveform decouples the excitation bandwidth from the pulse duration. [4] For Fourier transform rotational spectroscopy, the practical excitation pulse duration is limited by the dephasing processes for the subsequent FID. For the low-pressure (1-10 mTorr), room temperature gases used in this work, the Doppler effect is the limiting dephasing mechanism. The typical time scale, which depends on the square root of the mass/temperature ratio, is 1-3 $\mu$ s for molecules in the 20-120 amu mass range that are amenable to room temperature rotational spectroscopy.

The high-power AMC light source used in this work has more power than needed for direct absorption spectroscopy where source powers are usually kept in the 1-10 $\mu \mathrm{W}$ range to avoid saturation effects. [32] This peak power also exceeds the amount required to achieve optimal excitation using coherent single-frequency excitation on a time scale shorter than the dephasing limit. For example, the spectrometer used here can achieve this " $\pi / 2$-pulse" condition using a 400 ns pulse (sufficiently short compared to the $2 \mu \mathrm{s}$ 
dephasing time) for OCS with dipole moment of 0.712 D. [33] The chirped pulse distributes the source power over a wide measurement bandwidth and makes more efficient use of the high source power.

The decoupling of the excitation bandwidth from the pulse duration leads to an important distinction of the signals from chirped pulse excitation and single-frequency pulse, transform limited excitation. In the weak pulse limit, the signal strength for chirped pulse excitation is inversely related to the square root of the pulse bandwidth $\left(\alpha^{-1 / 2}\right)$, while the transform limited pulse excitation signal decreases linearly with the reciprocal of the bandwidth $\left(\alpha^{-1}\right)$ (see Fig. 1.2). [4] The behavior of the signal with chirped pulse excitation versus bandwidth has the result that, all things being equal, the measurement time to reach a target sensitivity level is independent of the number of spectrum segments used to cover the spectrometer operating range. This principle is founded upon the $\alpha^{-1 / 2}$ signal enhancement with reduced bandwidth and the $N^{1 / 2}$ noise floor reduction with the number of signal averages. For example, when splitting a broadband measurement into two equal bandwidth segments, the chirps concentrate the excitation power into a narrow frequency range so that signals will be larger by $2^{1 / 2}$. However, it takes twice as much time to address the entire band when segmenting the spectrum into two parts. The measurement which addresses the entire band in one segment can apply the factor of 2 in time to signal average and reduce the noise floor by a $2^{1 / 2}$ recovering the sensitivity gain from the segmented spectrum. The end result is an equal measurement time to reach equal sensitivity. Note that this scaling does not hold when the same scheme is applied for transform limited pulses, where performing a 


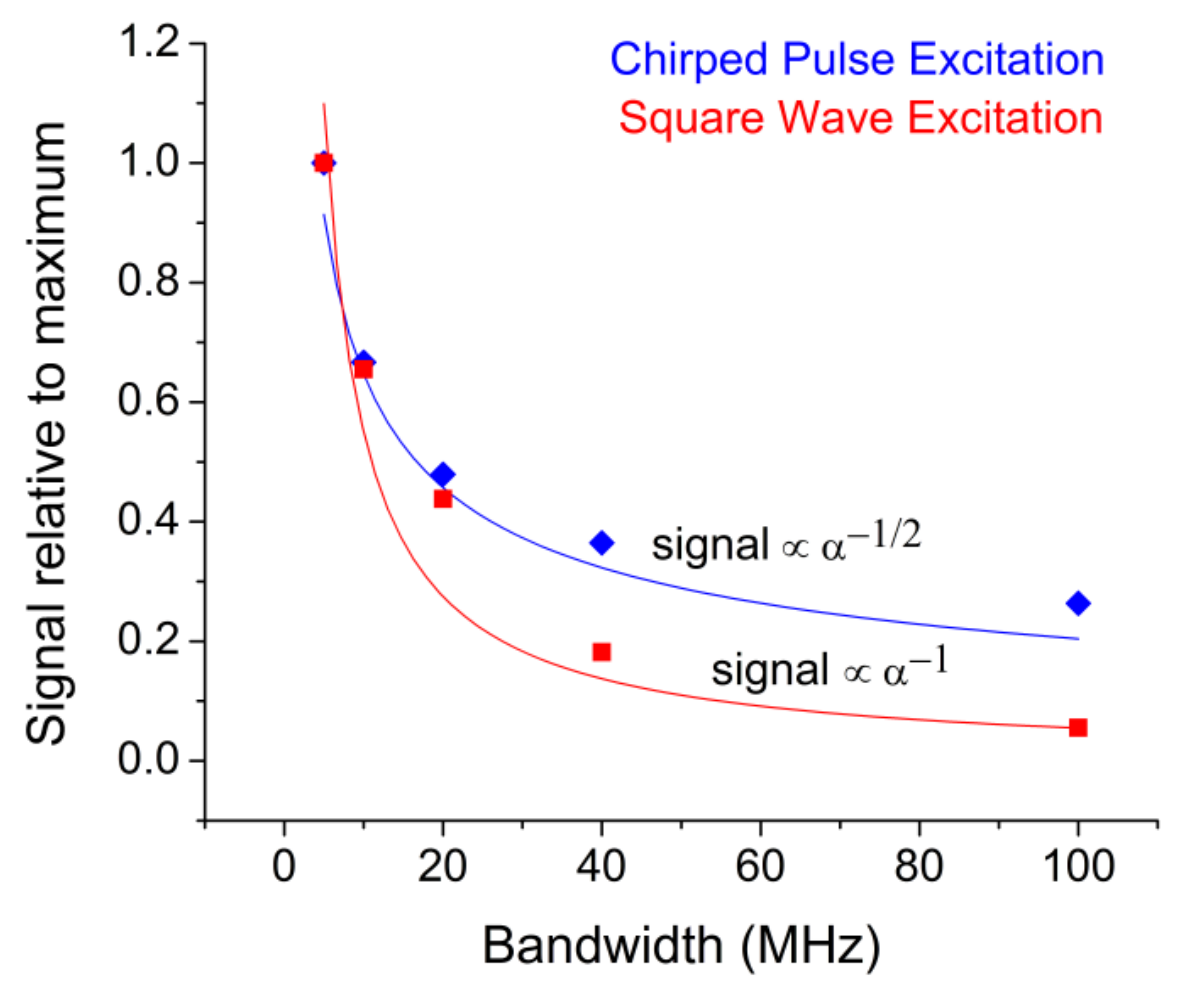

Figure 1.2: Signal drop as a function of bandwidth for chirped pulses at fixed duration (black) and for transform limited pulses (blue). The chirped pulse signals fall according to $\alpha^{-1 / 2}$ and the transform limited signals fall according to $\alpha^{-1}$, where $\alpha$ is the full width half max bandwidth of the excitation pulse. 
full band measurement with a single pulse will take two times longer than using two separate measurements with half the bandwidth.

In an instrument implementation, the molecular emission will be captured using a high-speed digitizer. All things being equal, segmenting the chirped pulse scan offers no advantages, but when the technical capabilities of the digitizer are considered, there can be significant performance and cost improvements because segmenting reduces the digitizer sampling rate requirement. In general, using $\mathrm{N}$ segments reduces the number of data points that need to be processed by a factor of N. Referring back to the two segment example, at half bandwidth, the sampling rate can be reduced by two. Summing the data points for two segments results in the same number of data points that would be collected in the full band measurement with double the sampling rate. However, to reach the same sensitivity, the "single segment" measurement needs to be acquired twice (leading to equal measurement times as pointed out above) so that twice as much data must be processed. There are also some data process performance gains in the FFT algorithm where the number of computations scales as $\mathrm{O}(n \log n)$, but these are likely offset by the extra processing required to concatenate the segment scans to produce the full spectrum.

The performance and cost benefits of segmented CP-FT spectroscopy are clear when measurements with $100 \%$ duty cycle are desired. For the current instrument design, the FID collection time is about $2 \mu \mathrm{s}$ and the full spectrometer bandwidth is $35 \mathrm{GHz}$. The minimum number of data points required to record a spectrum over the full frequency range is 140,000 (this measurement requires a digitizer operating at a minimum of $70 \mathrm{GS} / \mathrm{s}$ ). The digitizer used in our previous broadband design was an 8-bit, $100 \mathrm{GS} / \mathrm{s}$ digitizer in a digital oscilloscope. [28] The signal averaging rate for a $2 \mu$ s FID 
collection time of a $35 \mathrm{GHz}$ full band chirped measurement was $400 \mathrm{~Hz}$, a duty cycle of just $0.08 \%$. High-speed digitizers are commercial available with enough memory (512 kpts) in the FPGA to cover the instrument frequency range of $260-295 \mathrm{GHz}$ and signal average effectively in real time. Our spectrometer uses a 4 GS/s PCI digitizer card with FPGA signal accumulation capabilities that can achieve near $100 \%$ duty cycle measurements (each accumulation has a $1.8 \mu$ s re-arm time out of a maximum of $131 \mu \mathrm{s}$ acquisition time at $4 \mathrm{GS} / \mathrm{s})$.

The AWG waveform repeatedly loops through the excitation and LO waveforms without missed clock cycles. Because the segment durations are a multiple of the $10 \mathrm{MHz}$ reference clock period (100 ns) by design, successive waveforms have the same phase with respect to the PDRO clock in the up conversion circuit and the digitizer clock (2 GS/s). Therefore, accumulations of the full band scan are phase coherent and permit signal averaging. The AWG waveforms and data collection routine are customized for either high-speed or high dynamic range and are described in the operating modes section.

\section{Spurious Signals and Instrument Design}

A major challenge for broadband spectrometers is to minimize spurious signals that ultimately compromise the dynamic range of the measurement and complicate spectral assignment. For CP-FT mm-wave spectrometers, an important source of spurious signals is the AWG-driven AMC light source. In practice, we generate good quality chirped pulses with the AWG-based system and they are not a major influence to spectral purity. The detection principle is a significant difference between the CP- 
FTmmW design and traditional absorption spectrometers [32, 34-43] that detect the transmitted light from the mm-wave source and are, therefore, more sensitive to the spectral purity of the light source. CP-FT spectrometers detect the coherent molecular emission following excitation and this background-free molecular emission is spectrally pure. The main challenges for minimizing the spurious signals in CP-FT spectrometers are in the heterodyne receiver design.

\section{A. Spurious Signals from LO Impurity}

Because the CP-FT method requires an LO source that can switch frequencies instantaneously and advance to the next segment, the LO is generated using an AWG. The AWG LO output will have spurious signals in the bandwidth that get upconverted to the input range of the AMC chain. The most recent generation of high-speed AWGs have been tested in our system (the Agilent M8190a used in these measurements and the Tektronix AWG70000) and the spurious output has been reduced significantly compared to earlier units making the CP-FT design practical.

The LO spectral impurity contaminates the spectrum through two mechanisms during LO-to-IF conversion: 1) spurious signals mix in the mm-wave SHM resulting in signals within the IF detection bandwidth, and 2) LO spurious signals mix with molecular signals to produce spectrum images in the detection band. The first source of spurious signals can be corrected by making an electronic "background" measurement. This measurement takes place without chirped pulse excitation so that there is no molecular emission input to the mixer. Note that the molecular sample does not need to be removed from the cell so this method is not the same background correction that would be required 
in a dual beam absorption spectrometer. The electronic background of the LO can be subtracted to better than $1 \%$.

The second source of LO-to-IF conversion spurs only appear when the molecular emission signal is present, so there is no easy way to remove them. In the current instrument's high-speed mode, the molecular images tend to appear at about $1 \%$ of the real signal. The presence of these spurious signals is illustrated in Fig. 1.3 where the spectrum of acetonitrile acquired using an AWG generated LO is compared to a spectrum acquired using a high spectral purity microwave synthesizer (Agilent 83620B) as the LO source. In order to minimize spurious images, the AWG output is restricted to integer multiples of $30 \mathrm{MHz}$, which were determined to provide improved AWG spectral purity. The $30 \mathrm{MHz}$ AWG intervals multiply to $720 \mathrm{MHz}$ intervals after broadcast, and the IF band is chosen to be $720 \mathrm{MHz}-1440 \mathrm{MHz}$. The detection bandwidth is an important design parameter because it sets the minimum requirements on digitizer sampling rate to achieve real-time signal acquisition. Depending on the application, spurious signals at the $1 \%$ level may be intolerable. The high dynamic range mode, described later is designed to reduce this source of spurious signals.

\section{B. Spurious Signals in the Receiver Signal Path and from Data Processing}

There are several sources of spurious signals that can appear in the SHM, IF amplifier, and digitizer even in the case where there is an ideal LO signal. Nonlinearity in any of the components will produce intermodulation (IM) signals. For broadband detection where there are a large number of molecular signals present at once, the number of spurs from these sources can proliferate rapidly. The spectrometer design minimizes 


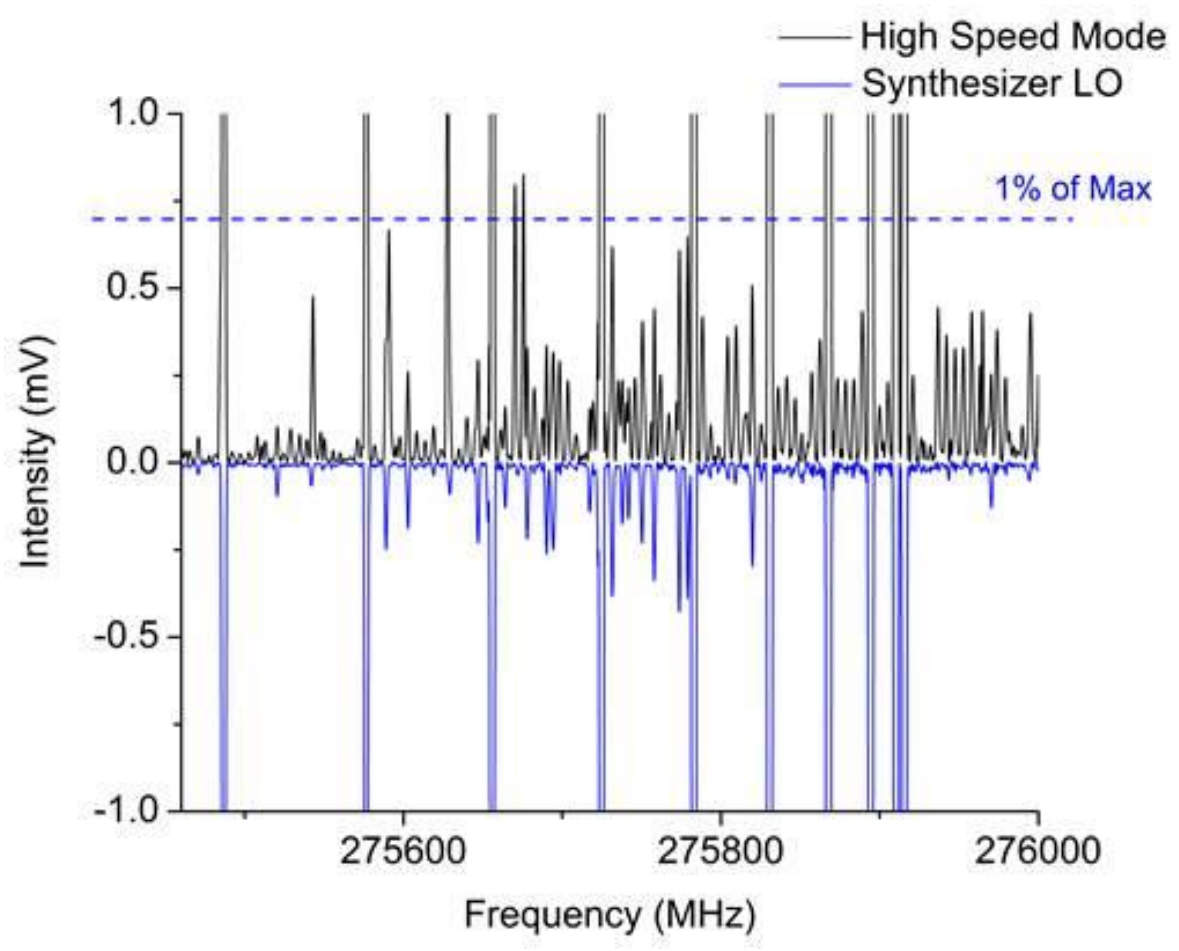

Figure 1.3: A 10,000 shot, $1 \mathrm{mTorr}$ spectrum of acetonitrile $(\mathrm{J}=15 \rightarrow 14)$ measured on the CP-FTmmW spectrometer. For acetonitrile, the image content in the spectrometer high-speed mode (black) confuses the spectrum at approximately the $1 \%$ level. The spurious signals occur in intervals that match the splitting of the two strongest lines at the high end of this spectrum (off scale, $70 \mathrm{mV}$ ). The spectral quality using the AWG driven LO is compared to the microwave synthesizer generated LO (blue). 
second order IM signals (sum and difference frequencies) by placing the detection band in the second octave - that is, the $720 \mathrm{MHz}$ segment bandwidth is detected from $720 \mathrm{MHz}$ - $1440 \mathrm{MHz}$. For an upper detection frequency of $1440 \mathrm{MHz}$, a digitizer with at least $2.88 \mathrm{GS} / \mathrm{s}$ (and FPGA signal accumulation capabilities) is required. This design condition was the basis of our choice of a $4 \mathrm{GS} / \mathrm{s}$ real-time digitizer.

Third order IM signals are more difficult to circumvent. These include the twotone IM signals where the beat frequency of two strong signals will modulate all other signals adding sidebands. The modulation can occur if the strong signals are out of the linear performance range of the mm-wave mixer or IF amplifier. The relative intensity of these spurious signals decreases with the input signal levels and have been observed for very strong signals as in Fig. 1.3 where many of the spurs have a $5.3 \mathrm{MHz}$ spacing corresponding to the splitting between the two strongest acetonitrile signals $\left(15_{0} \rightarrow 14_{0}\right.$ at 275915.6 $\mathrm{MHz}$ and $15_{1} \rightarrow 14_{1}$ at $275910.3 \mathrm{MHz}$ ). The third order IM spurs can be minimized by careful design of the IF amplification (described in the intensity calibration section) to include high IP3 performance specifications.

After the receiver, there can be spectrum images generated in the digitizer that come from mixing of the molecular signal with other clock signals on the digitizer board. High-speed digitizers interleave multiple core digitizers and imperfections in the interleaving can produce these spurs. For example, the $4 \mathrm{GS} / \mathrm{s}$ digitizer used in this instrument interleaves two 2 GS/s channels. Mixing of molecular signals in the $720-1440$ $\mathrm{MHz}$ band with this $2 \mathrm{GHz}$ clock will create images that overlap the detection band. This 
source of spurious signals appears to give small contributions, at most, to the signal detection.

Finally, there is another type of "spurious" effect that is related to the signal processing of the receiver system. The signal processing of the broadband FID uses a spectral window function to minimize the wings of the line shape - often called spectral leakage. This capability is important for room temperature rotational spectroscopy. To a good approximation, the decay of the FID is the product of two contributions: the Doppler dephasing (with $e^{-t^{2}}$ dependence) and collisional relaxation (with $e^{-t}$ dependence). When the frequency domain spectrum is produced, this functional form for the FID yields a Voigt line shape. The collisional relaxation contributes a Lorentzian line shape that increases with total sample pressure. The long Lorentzian wings hurt the baseline resolution of the measurement and, in most cases, degrade the dynamic range of the spectrum. A Kaiser-Bessel function with a variable $\beta$ parameter which serves to tighten the window that shapes the FID, offers a slight tradeoff in signal strength and FWHM for improved baseline resolution. [44] For analytical chemistry applications, the baseline line width is often the performance metric most closely tied to the measurement dynamic range because it characterizes the ability to detect a weak signal in close proximity to a strong signal. The sensitivity of CP-FT spectrometers for room temperature rotational spectroscopy is improved using this signal processing because high total gas pressure can be used without losing dynamic range. The use of the window function to improve base line resolution is illustrated in Fig. 1.4 where a narrowing of a Kaiser-Bessel window (beta parameter) reduces spectral leakage. 


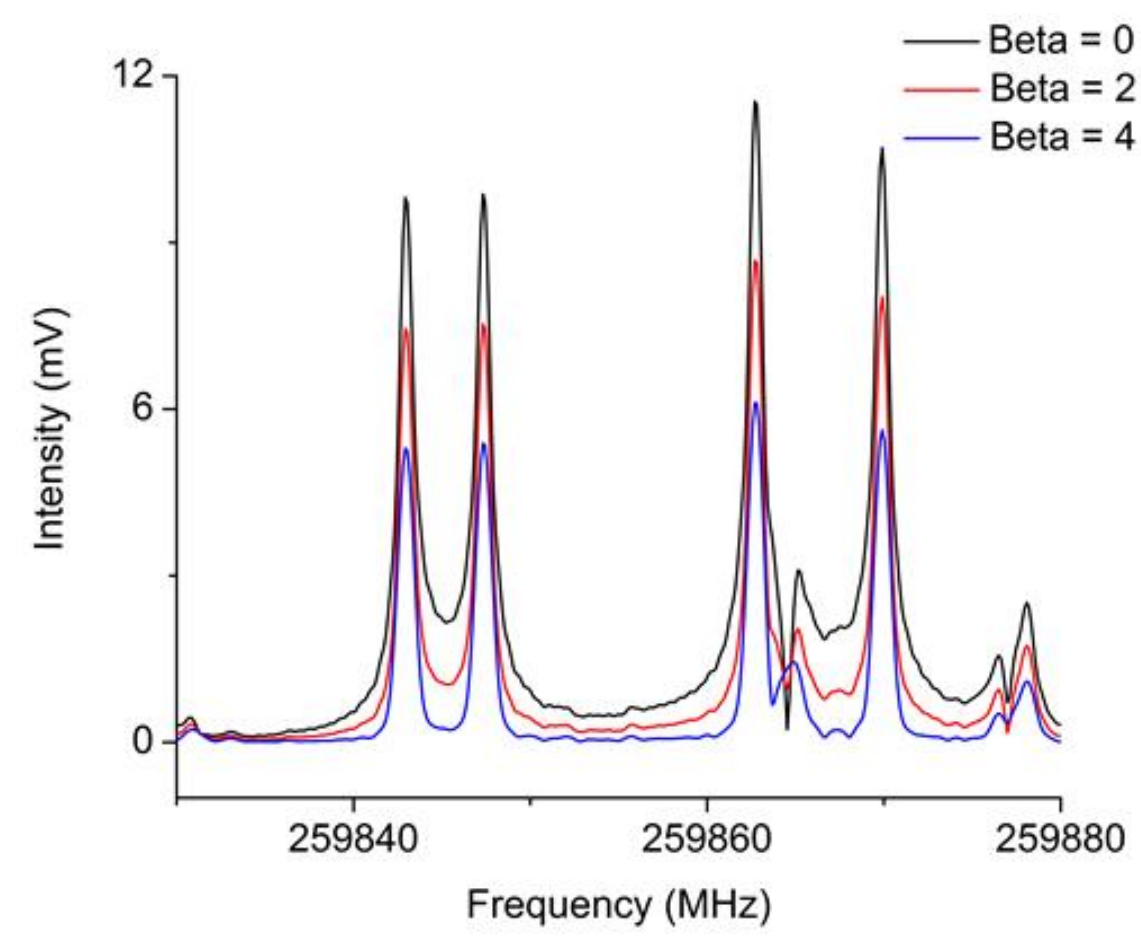

Figure 1.4: A 10,000 shot, 2 mTorr spectrum of ethyl cyanide measured on the CP-FTmmW spectrometer in HDR mode zoomed in on the horizontal scale. The baseline resolution improves as the beta parameter increases, narrowing the Kaiser-Bessel window function and enabling higher measurement pressure without a sacrifice in baseline resolution. 


\section{Operating Modes of the CP-FT mm-wave Spectrometer}

There are important tradeoffs in measurement speed (implying broadband excitation) and dynamic range (requiring low spurious signal content) for spectrometer operation. We have implemented two AWG-based measurement schemes that operate at the extremes of these two limits. The different operation modes simply use different excitation waveforms from the AWG and different acquisition modes of the digitizer so both modes can be used without any changes to the spectrometer components. High-speed operation makes use of broader band segments so that the entire band can be signal averaged within the memory depth of the accumulator. For high dynamic range operation, HDR mode minimizes spurs by utilizing reduced bandwidth segments. Intensity calibration is performed with the same basic routine, but with a corresponding structure in each mode.

\section{A. High-speed Operation}

The goal for high-speed operation is to obtain the rotational spectrum over the full spectrometer frequency range $(260-290 \mathrm{GHz})$ in as short a time as possible that still permits real-time spectrum acquisition. The key design parameters are the dephasing time and the memory depth of the FPGA used for signal accumulation. The FID will decay on a time scale of the Doppler dephasing or faster when the sample pressure becomes high enough that collisional relaxation is the dominant dephasing mechanism. Collisional relaxation rates are molecule and gas mixture dependent, but typically Doppler dephasing will dominate for total sample pressures below 10 mTorr at room temperature. For the size range of molecules that are well suited for room temperature 
mm-wave spectroscopy (about $100 \mathrm{amu}$ or lower), the Doppler dephasing takes place on a time scale of about $2 \mu \mathrm{s}$.

The basic segmented CP-FT concept involves acquiring a set of back-to-back, reduced bandwidth spectra. A total of 50 measurement segments are recorded to cover the $260-295 \mathrm{GHz}$ frequency range of the high-power AMC mm-wave source. Each measurement cycle is $2 \mu \mathrm{s}$ in duration, for a total measurement time of $100 \mu$ s (well within the $131 \mu$ s limit in FPGA memory depth at 4GS/s . Within each measurement cycle, a chirped excitation pulse with bandwidth $720 \mathrm{MHz}$ (30 MHz bandwidth in the AWG waveform) and a duration $250 \mathrm{~ns}$ is used to polarize the gas sample. The FID is acquired for the remaining $1.75 \mu$ s of the segment measurement time while the second channel of the AWG generates the single frequency LO to drive the receiver. There is approximately $50 \mathrm{~ns}$ of measurement dead time following the chirped excitation pulse as the receiver recovers from the high-power chirped pulse. In general, a duty cycle of close to $10 \%$ for the excitation pulse is chosen to avoid damage to the mm-wave SHM. The LO is chosen to be $720 \mathrm{MHz}$ below the chirped excitation pulse and is incrementally increased with each new segment. A full band measurement can be as fast as $100 \mu$ s and must be paired with an electronic background measurement that also takes $100 \mu \mathrm{s}$. However, depending on the nature of the experiment, the background measurement need only be recorded once across several data sets. A schematic figure illustrating the chirped pulse and LO AWG waveforms is shown in Fig. 1.5.

The high-speed, segmented CP-FT rotational spectrum of a 1 mTorr sample of acetonitrile $\left(\mathrm{CH}_{3} \mathrm{CN}\right)$ after 10,000 averages is shown in Fig. 1.3, and previously discussed. This figure shows the presence of a dense set of spectral features in the 


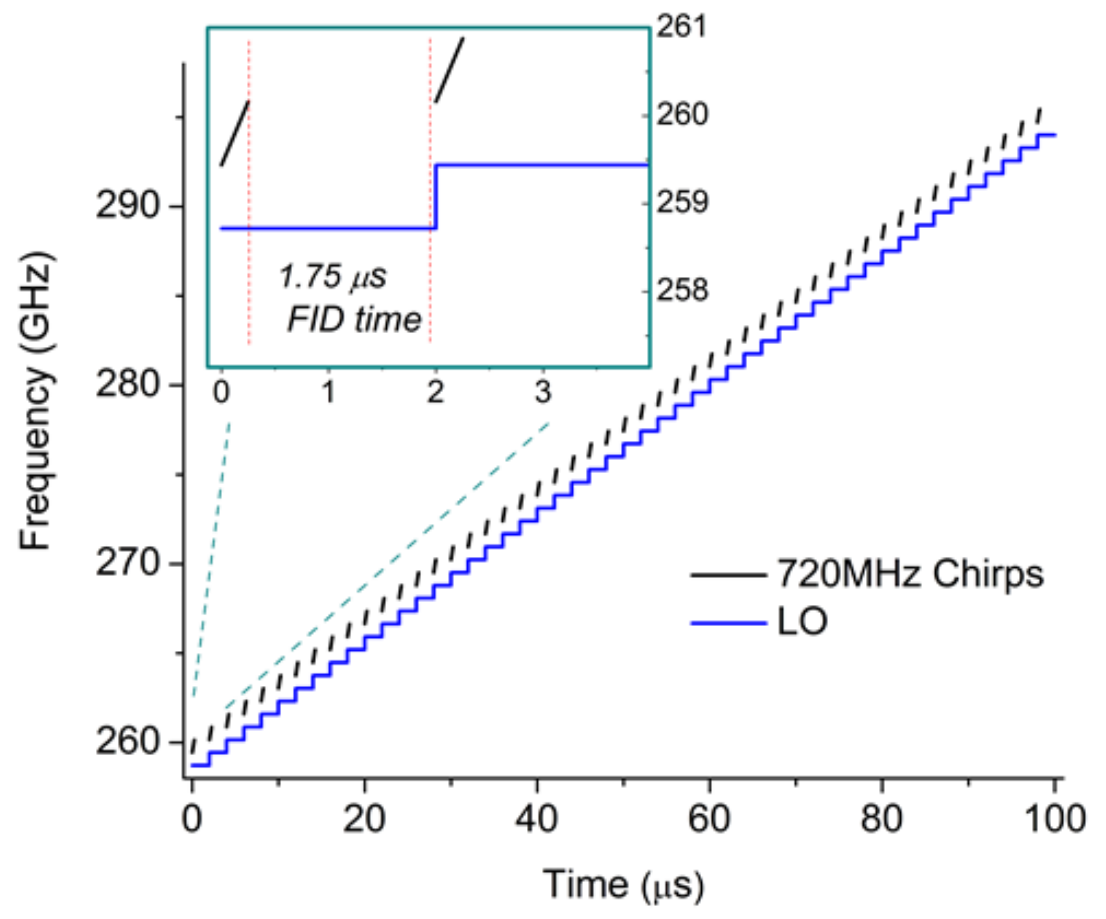

Figure 1.5: A conceptual spectrogram of the high-speed mode segmented chirped-pulse measurement protocol. 50 excitation/detection segments address the entire $35 \mathrm{GHz}$ bandwidth without overrunning the digitizer memory depth of the FPGA real-time accumulator. Each segment includes a $250 \mathrm{~ns} 720 \mathrm{MHz}$ chirp paired with a local oscillator, single frequency sine wave $2 \mu$ s in duration. The full band spectrum is acquired in $100 \mu \mathrm{s}$. 
frequency range where the strongest rotational transitions of acetonitrile occur. These features have amplitudes that are about 100-1000 times weaker than the strongest transitions. The vast majority of these signals are spurs. These spurious signals limit the dynamic range of the measurement in spectrum segments where strong transitions occur. Since these spurious signals are generated as images of the molecular transitions, they do not occur in other regions of the spectrum. This effect is the performance trade off that comes from achieving high-speed spectrum acquisition (full 260-295 GHz spectrum coverage in $100 \mu$ s with the ability to average the spectrum in real time).

\section{B. High Dynamic Range Operation}

The goal for high dynamic range operation is spectral accuracy for library quality spectra. HDR mode is a segmented chirped pulse measurement with individual chirp bandwidths sufficiently small to nearly remove all spurious images generated from the LO impurity in the SHM. The strongest LO spurs in the millimeter-wave spectrum generally occur on $30 \mathrm{MHz}$ intervals. If the segment bandwidth is less than $30 \mathrm{MHz}$, then the $30 \mathrm{MHz}$ spurs can contribute at most one image in the band for that particular segment. Such is the principle used to justify the HDR mode.

HDR mode makes further use of the AWG agility. Each segment has a measurement duration of $2 \mu \mathrm{s}$, as in high-speed mode, however the chirp bandwidth is chosen to be $24 \mathrm{MHz}$. The HDR measurement scheme can be understood in terms of splitting each $720 \mathrm{MHz}$ segment of the high-speed mode into 30 smaller segments that collectively make up the $720-1440 \mathrm{MHz}$ IF, each with the same LO. The measurement sequence is shown in the conceptual spectrogram in Fig. 1.6. The LO remains constant 


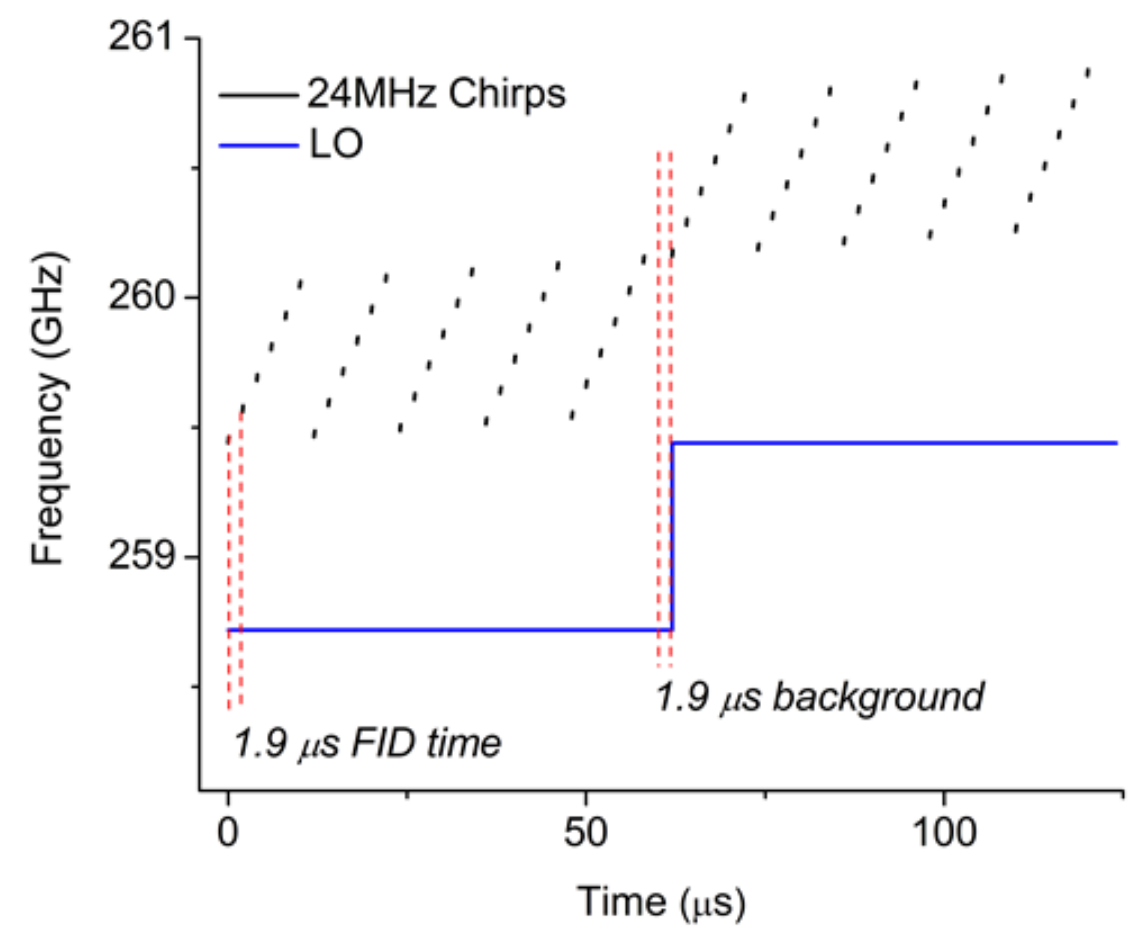

Figure 1.6: A conceptual spectrogram of the segmented chirped-pulse measurement protocol for HDR mode. The $720 \mathrm{MHz}$ chirp from high-speed mode is divided into 30, $24 \mathrm{MHz}$ chirps each paired with the same LO frequency. Thirty, $2 \mu \mathrm{s}$ excitation/detection sequences and one, $2 \mu$ sackground measurement requires half of the FPGA memory. $1440 \mathrm{MHz}$ of bandwidth can be acquired in real-time without overrunning the memory of the FPGA. Sequential excitation pulses are designed to be $120 \mathrm{MHz}$ apart in order to avoid bandwidth overlap between neighboring segments. 
throughout 30 HDR segments, which together address the equivalent of the high-speed $720 \mathrm{MHz}$ segment bandwidth. A $31^{\text {st }}, 2 \mu$ s segment without an excitation pulse is included for capturing the electronic background for that particular LO. As illustrated in the figure, the chirps do not step linearly in sequence. Consecutive sweeps in time are $120 \mathrm{MHz}$ apart in band in order to minimize power overlap. In contrast to high-speed mode, only $1440 \mathrm{MHz}$ of bandwidth is acquired with real-time signal averaging at a time in HDR mode. In total, a full-band acquisition in HDR mode requires 1500 segments or $3 \mathrm{~ms}$ of data. The AWG, with $2 \mathrm{GS}$ of memory, can hold a $160 \mathrm{~ms}$ long waveform in memory for continuous cycling, however, the digitizer only has $131 \mu$ s of memory in the FPGA accumulator at 4GS/s. So, the measurement is divided into 25 consecutive deep averaged data sets. The overhead for background measurement is not 1:1 as it is in highspeed mode where the $100 \mu$ s measurement is taken twice, once with the excitation pulses and once without for background subtraction. The LO sets the electronic background and, although the HDR chirps have 1500 unique bands, the LO waveform only changes 50 times. Out of $3 \mathrm{~ms}$, only $100 \mu$ s of the time is used for measuring the electronic background.

Although the minimum time to acquire an HDR spectrum is approximately 10 min for 100k shots, when deep averaging in HDR mode is considered, there is a minimal tradeoff in sensitivity between the two modes. Suppose HDR were implemented with the same sweep duration as the high-speed mode. All things being equal, the concept of segmented chirped pulse ensures equal sensitivity independently of how the measurement is segmented. A 100k average HDR spectrum at 6 minutes, compared to a 1.4 million average high-speed mode spectrum at 6 minutes, would have essential the 
same signal to ratio; however, the high-speed baseline would represent confusion from spectral impurity rather than the receiver noise level. The HDR mode performance improvement is illustrated in Fig. 1.7 and compares a $720 \mathrm{MHz}$ bandwidth section of acetonitrile generated from high-speed mode to that of HDR mode using the AWG based LO. Baseline spur confusion is clearly minimized in HDR mode. The effects of signal leakage are visible around the closely spaced doublet of the two strongest, off-scale transitions $(\mathrm{K}=0,1$ around $275900 \mathrm{MHz})$. A higher beta parameter in the Kaiser-Bessel window can be used to optimize the baseline resolution of this region. The IM3 spurs are also visible surrounding the doublet.

\section{Intensity Calibration and Spectral Accuracy}

Since the PDRO and the AWG are locked to a highly accurate frequency standard (10 $\mathrm{MHz}$ rubidium oscillator), the frequency calibration is achieved directly in the fast Fourier transform of the FID. However, there are several factors on both the front end and the back end of the spectrometer that influence the measured intensity of molecular signals. In order to produce library quality spectra, the measurement protocol must capture the effects of variable excitation power in the source, excitation pulse power envelopes, receiver signal compression, frequency dependent receiver gain, and delayed

molecular emission. Influencers to the spectrometer response in regards to signal intensity can be grouped into characteristics associated with excitation and detection.

On the front end, the excitation power varies with the AMC source power curve and the power envelope of the short excitation pulse. Overall, the AMC source power 


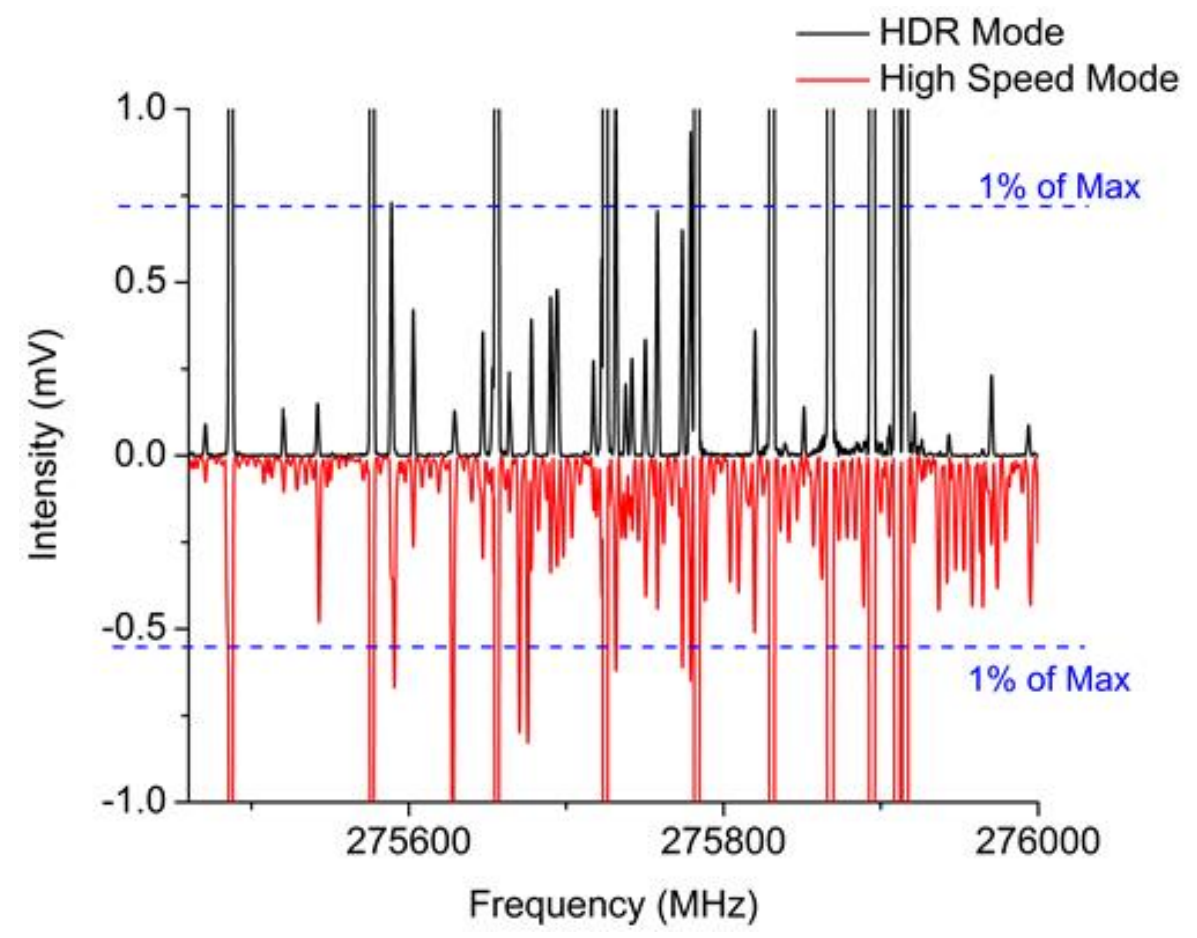

Figure 1.7: A 10,000 shot, 1 mTorr spectrum of acetonitrile measured on the CP-FTmmW spectrometer. For the HDR mode (black), the source power was attenuated from $14 \mathrm{~mW}$ to $1 \mathrm{~mW}$ in order to mitigate receiver saturation. The fast mode spectrum (red) is scaled up to match the signal height of the HDR spectrum. The signal-to-noise in the HDR spectrum is 350,000:1. For acetonitrile, the image content confuses the spectrum at approximately the $1 \%$ level. The spurious signals occur in intervals that match the splitting of the two strongest lines at the high end of this spectrum (off scale, $70 \mathrm{mV})$. 
peaks at the center of the band and falls toward the edges by as much as a factor of two. There are smaller fluctuations of 10-20\% within approximately every $5 \mathrm{GHz}$. The power broadcasted at a particular frequency is also largely dependent upon its relative frequency within the chirped-pulse. As the chirped-pulse bandwidth approaches the transform limited bandwidth, the power envelope of the chirp changes from a rectangular to sync form. In the limit that is nearly transform limited, frequencies toward the edges of the sweep can be underrepresented by $60 \%$ in power compared to the center. As a result, the excitation power curve has an overall shape matching the AMC source power curve and a more fine multi-pulse power envelope structure. This curve can account for most of the large amplitude intensity fluctuation of signals across the spectrometer band.

The back end influencers to signal intensity are IF signal compression and amplification which are dependent upon the receiver design. The SHM causes signal compression when the input approaches $100 \mu \mathrm{W}$. Strongly emitting molecules at high pressure can reach the compression limit of the SHM. Since this is an integral component to the receiver, the only way to compensate for the compression effect is to operate in conditions that reduce signal strength (for example, broader band excitation, attenuation at the source, or reduced pressure). Highly polar, symmetric tops and linear molecules may require low pressure or attenuated source power to ensure operation in the linear regime of the SHM. In addition to proper SHM input, careful selection of a low noise IF pre-amplifier after the SHM is also required to ensure linearity with receiver input power. The current spectrometer uses cascaded amplification in order to ensure wide dynamic range and preserve linear amplification of the strongest molecular signals. The first stage IF amplifier has a low noise figure $(0.4 \mathrm{~dB})$, a high P1-dB (15 dBm max 
output for linear gain) and high gain (20-25 dB). The second stage IF amplifier has a lower gain (10-15 dB) but similar P1-dB. Without sufficient pre-amplification, the low end of the dynamic range is limited by the digitization (the amplified noise must trip enough bits in the digitizer). With too much pre-amplification, the signals are subject to amplifier compression. In the current design, the dynamic range limiter is the 8-bit digitizer. Strong signals can be captured by extending the vertical voltage scale, however it is accompanied with a sacrifice in vertical resolution.

In the linear range of the receiver, the amplifier gain is another major contributor to signal intensity. Each of the IF amplifiers has a significant slope in the gain curve for the 720-1440 MHz band. As a result, signals which downconvert to low IF (towards $720 \mathrm{MHz}$ ) are amplified nearly twice as much as those at high IF (towards $1440 \mathrm{MHz}$ ). Finally, even if receiver response were totally linear, the FID represents a multiplexing of signals that start at different times. Transitions that are excited at the start of the chirped-pulse emit and decay for some time during the completion of the excitation pulse. As a result, the Fourier transform gate misses the strongest part of signals originating from the start of the chirp and intensities are underrepresented compared to later emitting transitions. In HDR mode, the pulse duration is reduced to $100 \mathrm{~ns}$ (compared to $250 \mathrm{~ns}$ in high-speed mode) in order to mitigate the deleterious effects of variable FID decays.

The frequency dependent effects can all be captured in one event. A spectrometer calibration curve is captured by broadcasting the same excitation waveform used for a broadband measurement (either high-speed or HDR), but at attenuated power so that the excitation pulses do not saturate the receiver. The excitation pulses are traced out on the digitizer and Fourier transformed, gating over the excitation pulse without any 
apodization in order to determine the excitation pulse power envelope for each pulse. Full band measurements thereafter are corrected with the normalized spectrometer response curve. The other effects such as compression and FID delay are mitigated by ensuring signal levels are below the saturation limit and by averaging two measurements in the frequency domain (one using negatively sweeping chirps, the other using positively sweeping chirps). Weekly comparison of calibration curves yields a reproducibility of approximately $2 \%$, which is likely dependent upon response from the manually dialed source attenuator.

\section{Results}

The CP-FT millimeter wave spectrometer performance is benchmarked against a 298K, ethyl cyanide $\left(\mathrm{CH}_{3} \mathrm{CH}_{2} \mathrm{CN}\right)$ spectrum published by Fortman et al., representing modern direct absorption spectroscopy. [45] The important experimental conditions to consider are measurement bandwidth, acquisition time, path length, and pressure. The direct absorption data set spans $60 \mathrm{GHz}$ (from $210-270 \mathrm{GHz}$ ) and required 40 seconds of acquisition time. Ethyl cyanide signals are approximately 400:1 using a $6 \mathrm{~m}$ path length and a pressure of 0.5 mTorr. For direct comparison, the CP-FTmmW bandwidth overlaps with the Fortman et al. results from $260-270 \mathrm{GHz}$. The overlapping band is a fair comparison in that it is at the edge of performance for both spectrometers; sensitivity improves towards the center of each spectrometer's band. All CP-FTmmW full-band spectra span $260-295 \mathrm{GHz}$ and were generated in a $65 \mathrm{~cm}$ path length sample cell with $3 \mathrm{~dB}$ beam diameter of approximately $2 \mathrm{~cm}$. The phase stability of the system permits averaging to the accumulation limit of the FPGA (16 million) without loss of signal. In 
empty cell measurements, the noise is observed to decrease as $\mathrm{N}^{1 / 2}$ out to the accumulation limit. In practice, the room temperature mm-wave spectrum of polyatomic molecules the size of ethyl cyanide and larger reach a practical confusion limit after a relatively small number of averages $\left(10^{4}\right)$.

The goal of the high-speed mode is to acquire broadband spectra in as fast a time frame as possible in order to capture time resolved events. In order to preserve full-band speed, an intensity calibrated spectrum does not include a sweep up/down average. The measurement precision was measured by subtracting two successive 10,000 shot (one second) ethyl cyanide spectra and is better than $0.5 \%$. A $100 \mu \mathrm{s}$, single shot, ethyl cyanide spectrum at $0.5 \mathrm{mTorr}$ is illustrated in Fig. 1.8. The signal-to-noise ratio is approximately 20:1 and the ethyl cyanide pattern is clearly discernible. After scaling for path length, bandwidth, and time, the measurement speed of the CP-FTmmW is 25,000 times faster (includes background subtraction) than the modern absorption spectrometer. That is, by CP-FTmmW a 400:1, $35 \mathrm{GHz}$ spectrum can be achieved with 6 meters of pathlength in $800 \mu \mathrm{s}$, whereas the same takes 23 seconds by the absorption technique. In terms of minimum detectable pressure of ethyl cyanide for 23 seconds of measurement time, CP-FTmmW is 160 times more sensitive (25 nTorr compared to $4 \mu$ Torr by Fortman et al.).

Since HDR mode is designed for library quality spectra, it is the mode of choice for comparison of spectral purity and intensity accuracy to the absorption technique. The HDR ethyl cyanide spectrum shown in Fig. 1.9 was measured at 2 mTorr and is a 10,000 shot time average, which takes approximately 70 seconds to acquire (30 seconds of signal averaging and 40 seconds of overhead in the AWG for transition between waveforms, a 


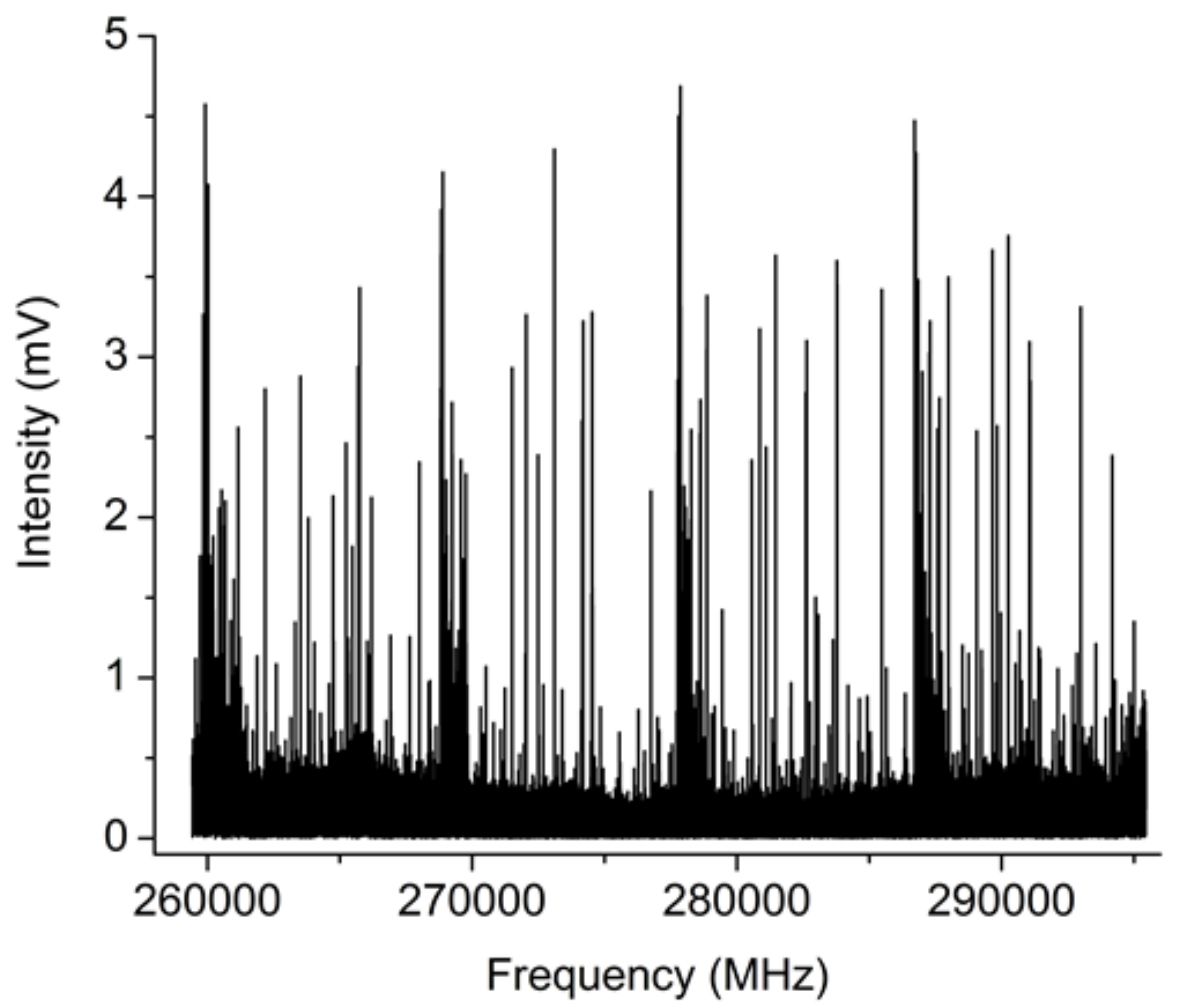

Figure 1.8: A single shot, 0.5 mTorr spectrum of ethyl cyanide measured on the $\mathrm{CP}-\mathrm{FTmmW}$ spectrometer in high-speed mode. The spectrum required $100 \mu \mathrm{sec}$ of acquisition time and has a signal-to-noise ratio of 20:1. 


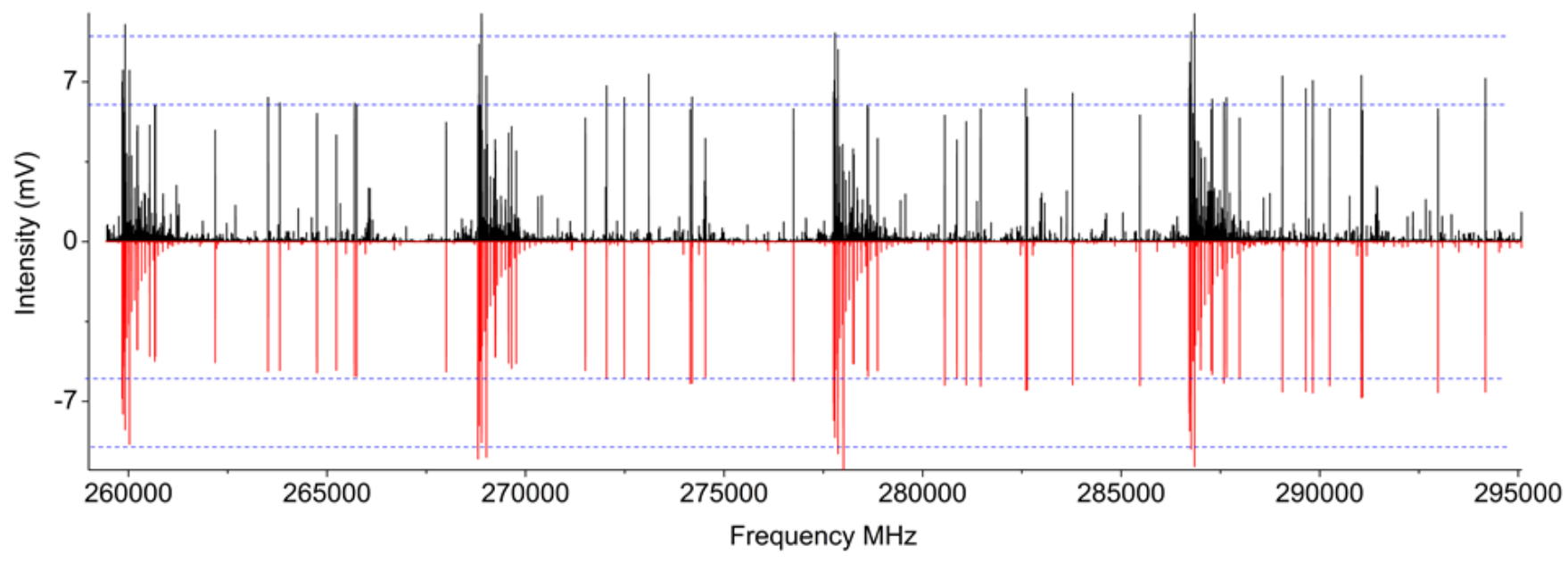

Figure 1.9: A 10,000 shot, 2 mTorr spectrum of ethyl cyanide measured on the CP-FTmmW spectrometer (black) in HDR mode. The relative intensity matches the simulation (red) illustrating the feasibility of using intensity profiles as a parameter for identification. The $1 \sigma \mathrm{rms}$ difference between the measured CP-FTmmW spectrum and the simulation is approximately $12 \%$. 
"sequencer" option in the AWG would make this transfer instantaneous). The CP-FTmmW measurement makes use of the improved sensitivity from higher total pressure ( 2 mTorr vs 0.5 mTorr) measurements enabled by the signal processing capabilities discussed previously which maintain baseline resolution. Above 2 mTorr the pressure broadening begins to dominate and the ethyl cyanide signal no longer increases linearly with pressure. The CP-FTmmW spectrum is a good match to the simulated ethyl cyanide pattern. A global fit of the ground state ethyl cyanide spectrum combining previously published line lists with the CP-FTmmW line list yields an RMS error of 80 $\mathrm{kHz}$, well within the established standard of accuracy for absorption methods. [46] An analysis of fit can be found in the supplementary material at the end of Chapter 1.

In Fig. 1.10, the Fortman et al. ethyl cyanide spectrum is scaled up in intensity to match the CP-FTmmW HDR spectrum so that the signal-to-noise ratio can be evaluated. The baseline x20 expansion of the intensity axis illustrates the spectral purity of the CPFTmmW library spectrum if the Fortman et al. spectrum is taken to be the spur-free reference. In a region of the strongest ethyl cyanide signals, where spurious images should be the most prevalent, the baseline is clean. Care should be taken when comparing weak signals in the two spectra. Baseline spline processing in the Fortman et al. dataset can cause the effect of removing weak lines. The intensity expanded view in Fig. 1.10 also illustrates the comparative noise level. The 10,000 shot HDR spectrum acquisition time is comparable to the absorption scan, but has 50 times the signal-to-noise ratio of 20,000:1 versus 400:1. The dual, sweep-up/sweep-down measurement and the proportionally larger unused bandwidth edges for each excitation chirp contribute to an HDR mode speed that is approximately half that of high-speed mode. The noise level, 


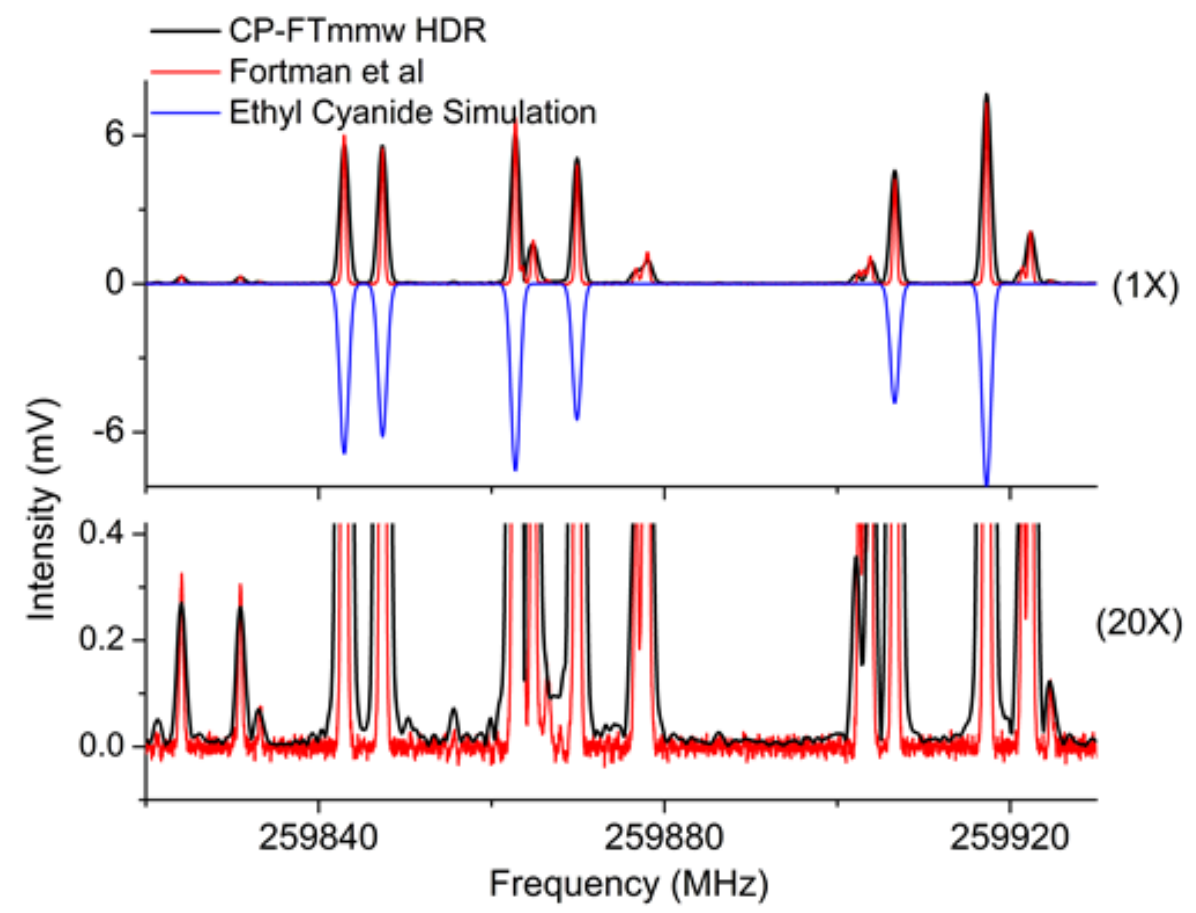

Figure 1.10: A 10,000 shot, 2 mTorr spectrum of ethyl cyanide measured on the CP-FTmmW spectrometer (black) in HDR mode. The relative intensity matches well to the simulation (blue). For nearly equal time/bandwidth, and scaled for pressure and path length the CP-FTmmW measurement has 50 times greater sensitivity over the absorption spectrum (red). Noise levels are illustrated in the bottom panel. The FWHM linewidths for CP-FTmmW are 2.5 times greater than the direct absorption method for Doppler limited pressures. 
however, cannot be directly assessed in the CP-FTmmW ethyl cyanide spectrum, which is confusion limited at the $0.1 \%$ level and limits the dynamic range to 1000:1. The spectrometer noise level is measured on a blank sample cell and is verified in the spectra of simpler molecules (like OCS) to be approximately $400 \mathrm{nV}$.

Although there is a significant gain in sensitivity, there are some tradeoffs to the CP-FTmmW speed when comparing other spectral parameters. The top panel of Fig. 1.10 illustrates the overall intensity accuracy of the two ethyl cyanide spectra compared to simulation. Lines that appear in the measured spectra and do not appear in the simulation can be attributed to different vibrational states of the molecule; the two measured spectra are in agreement for these lines. Without calibration of the CPFTmmW spectrum, pattern matching would be difficult. A histogram plot in Fig. 1.11 illustrates the reduction in fractional error after the intensity calibration. A least squares fitting algorithm was used to scale the overall intensity of the simulated spectrum to the measured spectrum while minimizing the $1 \sigma \mathrm{rms}$ and the average difference between line intensities. The line list for the scaling routine included only those that are believed to have minimal overlap (i.e., line strength within a factor of 10 of the strongest lines and no closely spaced doublets). Without applying the correction, the error distribution has essentially no convergence. After calibration, the $1 \sigma$ rms error between the CP-FTmmW library spectrum and simulation is approximately $12 \%$. The Fortman et al. results are also included on the histogram plot with 3 times better intensity performance ( $1 \sigma \mathrm{rms}$ of approximately 4\%).

Fig. 1.10 also highlights resolution differences. Since the spectrum is a gated magnitude transform, the typical FWHM linewidth by CP-FTmmW is $1.24 \mathrm{MHz}$, 


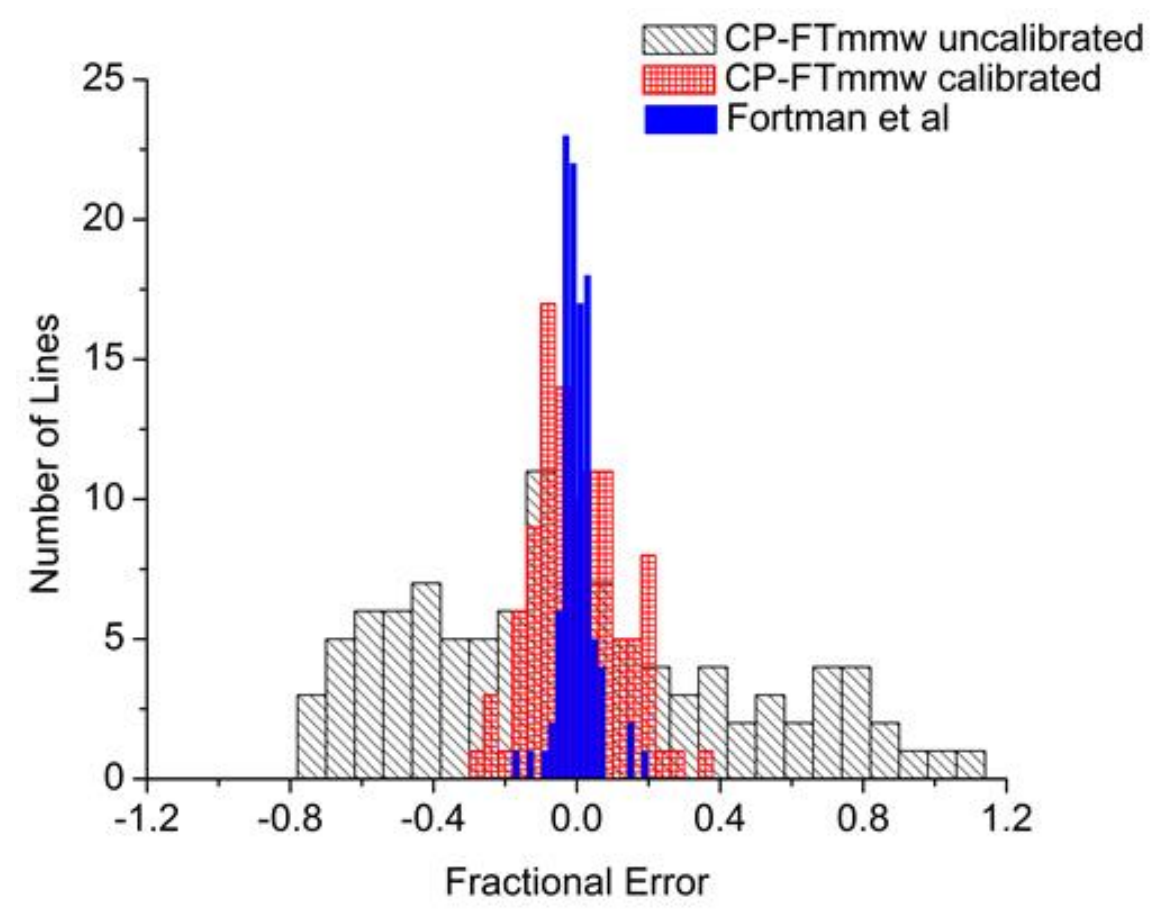

Figure 1.11: A histogram plot of fractional error between measurement and simulation for approximately 100 of the strongest lines in the ethyl cyanide spectrum. Without calibration (black) pattern matching is difficult, however, after calibration (red) 12\% intensity accuracy can be expected for the CP-FTmmW spectrometer. The fastest direct absorption technique performs with better than $4 \%$ intensity accuracy. 
approximately 2.5 times greater than the Doppler limited absorption line width (500 $\mathrm{kHz}$ ). CP-FTmmW linewidths are very consistent across the band and across a large dynamic range because the lineshape is largely influenced by the apodization function (Kaiser-Bessel, $\beta=6$ for these results) and Fourier transform gate used in signal processing. In the Fig. 1.10 overlay, the expanded view shows the baseline resolution of the $0.5 \mathrm{mT}$ Torr absorption spectrum and the $2 \mathrm{mTorr}$ CP-FTmmW spectrum. At 4 times the total pressure, the CP-FTmmW still displays a sharp fall to baseline more similar to Gaussian behavior rather than Lorentzian. This behavior is a unique advantage over the absorption spectrum broadening which can only be addressed by working at lower total pressure. Although the minimum detectable pressure is not affected, the minimum detectable concentration is improved by CP-FTmmW with the capability of measuring mixtures at pressures beyond the Doppler limit. A trace analyte in a predominantly nitrogen sample matrix, for example, could be measured in a total pressure of perhaps 100 mTorr versus 1 mTorr, resulting in 2 orders of magnitude better concentration detection limit.

In order to illustrate the dynamic range that is not confusion limited, a library spectrum of OCS was measured in HDR mode. OCS isotopologues in natural abundance down to ${ }^{18} \mathrm{O}^{13} \mathrm{CS}$ (natural abundance $0.00211 \%$ or $1 / 48,000$ relative to the main isotopologue) can be detected with a signal-to-noise of greater than 5:1 in the 100,000 shot, 12 minute measurement. The five orders of magnitude of linear dynamic range is verified in Fig. 1.12 which shows a log plot of the abundance versus line intensity for three lines each of 11 isotopologues of OCS with a clear detection of ${ }^{18} \mathrm{O}^{13} \mathrm{CS}$ illustrated 


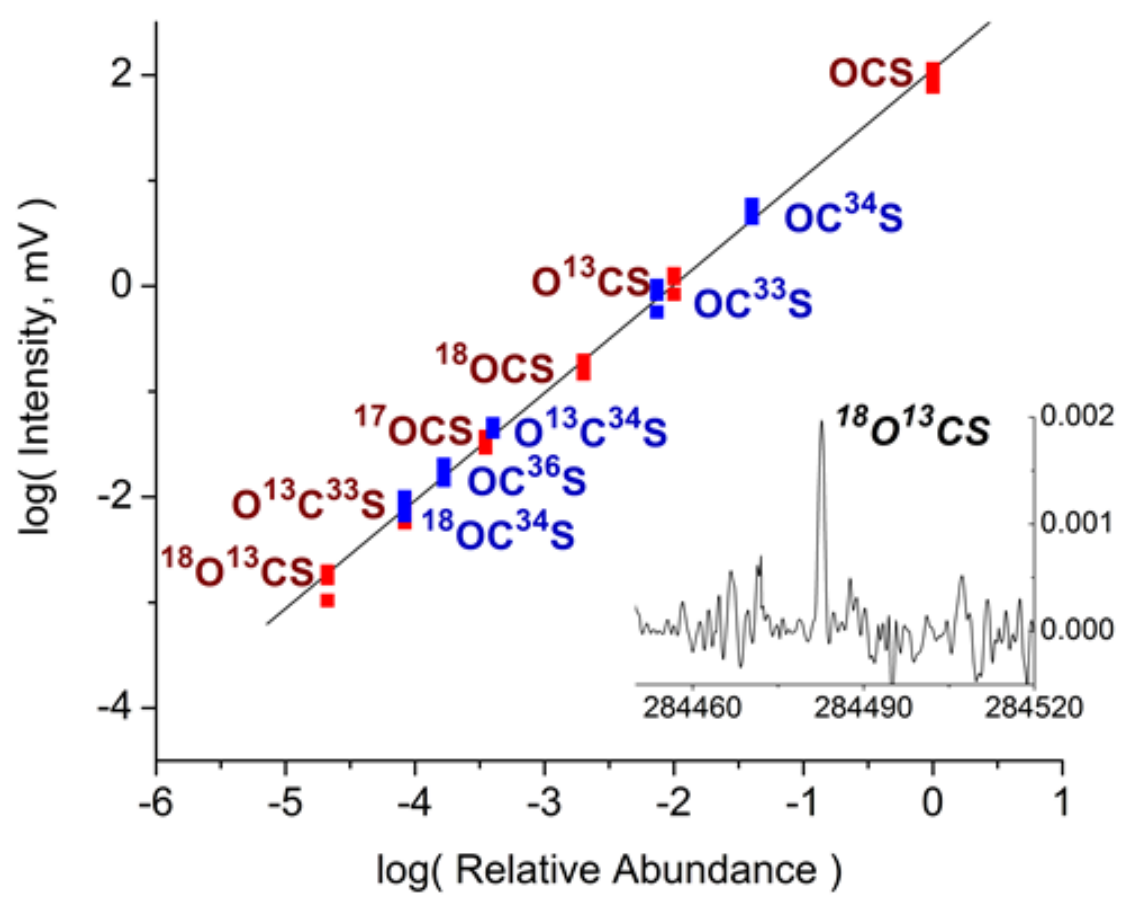

Figure 1.12: A logarithmic plot of OCS isotopologue relative abundance versus signal in a 100,000 shot, 3 mTorr spectrum of OCS measured on the CP-FTmmW spectrometer in $\mathrm{HDR}$ mode. The five orders of magnitude in dynamic range reach the ${ }^{18} \mathrm{O}^{13} \mathrm{CS}$ isotopologue with a natural abundance $1 / 48,000$ of the main isotopologue at a signal-to-noise of approximately 5:1. Three lines for each isotopologue are measured and contribute to the points on the plot. 
in the inset. The assignments, especially of the weakest lines, rely on the intensity trend to increase statistical confidence due to the simplicity of the linear rotor pattern which generates only three isolated OCS transitions across the band for each isotopologue. Fig. 1.12 illustrates the match between relative signal intensity and relative natural abundance across eleven isotopologues.

\section{Conclusion}

A broadband, CP-FTmmW spectrometer design has been presented and benchmarked with the routine use case of broadband acquisition for an unbiased spectral snapshot of a room temperature gas sample with either high-speed, or high dynamic range performance. The enhanced sensitivity of CP-FT over direct absorption and the software controlled, solid-state design promises new utility to research communities in the academic and industrial sectors. The versatility of CP-FTmmW is enabled by the use of the AWG that can be used to design customized waveforms and by the flexible, fast memory structure of the digitizer. When optimized for speed, the full band spectrum can be acquired on short enough time scales for monitoring dynamic systems in micro second time scales. Depending on how much bandwidth is required, the experiment time runs from $2-100 \mu$ s. In a $10 \mathrm{~Hz}$ laser duty cycle, the CP-FTmmW spectrometer could acquire 1000 full band shots per laser pulse yielding an ethyl cyanide detection limit of $2 \mu$ Torr.

Although the CP-FT technique is subject to spectral purity challenges, a high dynamic range mode has been presented which produces high sensitivity, library quality spectra in minutes. Broadband pattern matching of measured spectra to a library database 
(either a dynamic instrument specific library or from an external database) can speed the analysis of broadband datasets where the composition is not completely catalogued. Suspect molecular carriers in mixtures can be investigated quickly. Under software control the spectrometer can also be reconfigured for reduced bandwidth measurements in order to increase sensitivity for specific species of interest. A typical protocol might involve a broadband screen followed by a targeted search. A deep averaged targeted search could monitor several lines with real-time accumulation and have an excitation pulse tailored for optimal excitation of each transition (a single frequency $\pi / 2$ pulse). Other conventional Fourier transform methods which utilize the time resolved nature of excitation and detection can also be explored for more advanced analysis of mixtures. The advanced analysis routines developed for unknown identification and false positive reduction will be the basis of another publication.

Compared to other CP-FT techniques, the segmented CP-FTmmW spectrometer is the first real-time instrument (98\% measurement duty cycle) and realizes the sensitivity of CP-FT to its full potential at millimeter-wave frequencies, far exceeding that of modern absorption techniques. In addition to wide applicability for basic science research, CP-FTmmW makes rotational spectroscopy a competitive analytical instrument for gas composition analysis. Spectroscopists have long recognized the superior dynamic range and selectivity characteristics of rotational spectroscopy. As the technology advances, the cost of the current system will become more and more economical. There is also some freedom with current technology to reduce form factor and cost while sacrificing some utility in order to meet customized needs. 


\section{References for Chapter 1:}

1. R. H. Hughes, E. B. Wilson, Phys. Rev., 1947, 71, 562.

2. A. H. Sharbaugh, Rev. Sci. Instr., 1950, 21, 120.

3. G. G. Brown, B. C. Dian, K. O. Douglass, S. M. Geyer, B. H. Pate, J. Mol. Spec., 2006, 238, 200.

4. G. G. Brown, B. C. Dian, K. O. Douglass, S. M. Geyer, S. T. Shipman, B. H. Pate, Rev. Sci. Instr., 2008, 79, 053103.

5. C. Townes and A. Schawlow, Microwave Spectroscopy, 1975, Dover.

6. W. Gordy and R.L. Cook, Microwave Molecular Spectra, 1984, John Wiley and Sons.

7. J. K. G. Watson, A. Roytburg, W. Ulrich, J. Mol. Spec., 1999, 196, 102.

8. D. Patterson, M. Schnell, J. M. Doyle, Nature, 2013, 497, 475.

9. M. Goswami, J. L. Neill, M. Muckle, B. H. Pate, E. Arunan, J. Chem. Phys., 2013, 139, 104303.

10. C. Callegari, I. Reinhard, K. K. Lehmann, G. Scoles, K. Nauta, R. E. Miller, J. Chem. Phys., 2000, 113, 4636.

11. K. O. Douglass, J. E. Johns, P. M. Nair, G. G. Brown, F. S. Rees, B. H. Pate, J. Mol. Spec., 2006, 239, 29.

12. M. Nakajima, Y. Sumiyoshi, Y. Endo, Rev. Sci. Instru., 2002, 73, 165.

13. S. K. Witonsky, M. R. Canagaratna, S. L. Coy, J. I. Steinfeld, R. W. Field, A. A. Kachanov, J. Chem. Phys., 2001, 115, 3134.

14. D. J. Nesbitt, R. W. Field, J. Phys. Chem., 1996, 100, 12735.

15. K. Prozument, R. G. Shaver, M. A. Ciuba, J. S. Muenter, G. B. Park, J. F. Stanton, H. Guo, B. M. Wong, D. S. Perry, R. W. Field, Faraday Discuss., 2013, 163, 33. 
16. B. C. Dian, G. G. Brown, K. O. Douglass, F. S. Rees, J.E. Johns, P. Nair, R. D. Suenram, B. H. Pate, PNAS, 2008, 105, 12696.

17. B. H. Pate, J. Chem. Phys., 1990, 110, 1990.

18. K. O. Douglass, B. C. Dian, G. G. Brown, J. E. Johns, P. M. Nair, B. H. Pate, J. Chem. Phys., 2004, 121, 6845.

19. A. A. Makarov, Opt. Spectrosc., 1987, 62, 697.

20. B. H. Pate, J. Chem. Phys., 1998, 109, 4396.

21. D. Green, R. Holmberg, C. Y. Lee, D. A. McWhorter, B. H. Pate, J. Chem. Phys., 1998, 109, 4407.

22. K. Prozument, A. P. Colombo, Y. Zhou, G. B. Park, V. S. Petrović, S. L. Coy, R. W. Field, Phys. Rev. Let., 2011, 107, 143001.

23. D. Zaleski, J. Neill, M. Muckle, N. Seifert, P. Carroll, S. Widicus-Weaver, B. Pate, J. Mol. Spec.,2012, 280, 68.

24. G. S. Grubbs, C. T. Dewberry, K. C. Etchison, K. E. Kerr, S. A. Cooke, Rev. Sci. Instr., 2007, 78, 096106.

25. S. L. Stevens, N. R. Walker J. Mol. Spec., 2010, 263, 27.

26. D. A. Obenchain, A. A. Elliott, A. L. Steber, R. A. Peebles, S. A. Peebles, C. J. Wurrey, G. A. Guirgis, J. Mol. Spec., 2010, 261, 35.

27. R. G. Bird, J. L. Neill, V. J. Alstadt, J. W. Young, B. H. Pate, D.W. Pratt, J. Phys. Chem. A, 2011, 115, 9392.

28. A. L. Steber, B. J. Harris, J. L. Neill, B. H. Pate, J. Mol. Spec., 2012, 280, 3.

29. J. L. Niell, B. J. Harris, A. L. Steber, K. O. Douglas, D. F. Plusquellic, B. H. Pate, Opt. Expres., 2013, 21, 19743.

30. E. Gerecht, K. Douglass, D. Plusquellic, Opt. Lett., 2011, 19, 8973. 
31. G. B. Park, A. H. Steeves, K. Kuyanov-Prozument, J. L. Neill, R. W. Field, J. Chem. Phys., 2011, 135, 024202.

32. I. R. Medvedev, C. F. Neese, G. M. Plummer, F. C. De Lucia, Opt. Lett., 2010, 35, 1533.

33. S. M. Muenter, J. Chem Phys., 1968, 48, 4544.

34. B. J. Drouin, F. W. Maiwald, J. C. Pearson, Rev. Sci. Instr., 2005, 76, 093113.

35. C. Neese, I. Medvedev, G. Plummer, A. Frank, C. Ball, F. De Lucia, IEEE Sensors J., 2012, 12, 2565.

36. C. P. Endres, F. Lewen, T. F. Giesen, S. Schlemmer, D. G. Paveliev, Y. I. Koschurinov, V. M Ustinov, A. E. Zhucov, Rev. Sci. Instr., 2007, 78, 043106.

37. R. A. Motiyenko, L. Margules, E. A. Alekseev, J. C. Guillemin, J. Demaison, J. Mol. Spec., 2010, 264, 94.

38. Z. Kisiel, E. B. Jaworska, L. Pszczolkowski, J. Mol. Spec., 1996, 177, 240.

39. S. Saito, M. Goto, ApJ, 1993, 410, L53.

40. C. A. Gottlieb, P. C. Myers, P. Thaddeus, ApJ, 2003, 588, 655.

41. T. Okabayashi, T. Mitsutoshi, J. Chem. Phys., 1993, 99, 3268.

42. M. Winnewisser, H. Lichau, F. Wolf, J. Mol. Spec., 2000, 202, 155.

43. L. M. Ziurys, W. L. Barclay, M. A. Anderson, D. A. Fletcher, J. W. Lamb, Rev. Sci. Instr., 1994, 65, 1517.

44. J. F. Kaiser, Proc. IEEE International Simposium on Circuits and Systems, April 1974, pp. 20.

45. S. M. Fortman, I. Medvedev, C. Neese, F. C. De Lucia, ApJ., 2010, 725, 1682.

46. H. M. Pickett, R. L. Poynter, E. A. Cohen, M. L. Delitsky, J. C. Pearson, and H. S. P. Muller, J. Quant. Spectrosc. Radiat. Transf., 1998, 60, 883. 
Supplementary Table 1.1: Rotational constants measured from a JPL global fit of Ethyl Cyanide lines where the 260-295 GHz range is displaced with new CP-FTmmW data. The constants determined between the two fits agree to well within the uncertainty of the fit. The average OMC (observed - calculated) from $260-295 \mathrm{GHz}$ is $0.049 \mathrm{MHz}$ where the CP-FTmmW line list is used for the global fit compared to $0.039 \mathrm{MHz}$ for unaltered JPL data.

\begin{tabular}{|c|c|c|c|c|c|}
\hline & & CP-FTmmW global ${ }^{\mathrm{a}}$ & JPL global & $\sigma(\mathrm{JPL}$ global $)$ & $\begin{array}{l}\text { Magnitude } \\
\text { Difference }\end{array}$ \\
\hline $\mathrm{A}$ & $/ \mathrm{MHz}$ & $27663.68320(49)$ & $27663.68305(48)$ & $4.80 \mathrm{E}-03$ & $1.50 \mathrm{E}-04$ \\
\hline B & $/ \mathrm{MHz}$ & $4714.213397(76)$ & $4714.213343(75)$ & $7.50 \mathrm{E}-03$ & $5.40 \mathrm{E}-05$ \\
\hline $\mathrm{C}$ & $/ \mathrm{MHz}$ & $4235.059699(71)$ & $4235.059643(70)$ & $7.00 \mathrm{E}-03$ & $5.60 \mathrm{E}-05$ \\
\hline DJ & $/ \mathrm{kHz}$ & $3.073554(34)$ & $3.073531(34)$ & $3.40 \mathrm{E}-05$ & $2.30 \mathrm{E}-05$ \\
\hline DJK & $/ \mathrm{kHz}$ & $-47.65816(36)$ & $-47.65818(36)$ & $3.60 \mathrm{E}-04$ & $2.00 \mathrm{E}-05$ \\
\hline DK & $/ \mathrm{kHz}$ & $548.1257(28)$ & $548.1249(28)$ & $2.80 \mathrm{E}-03$ & $8.00 \mathrm{E}-04$ \\
\hline delJ & $/ \mathrm{kHz}$ & $-0.6859953(98)$ & $-0.6859957(95)$ & $9.50 \mathrm{E}-03$ & $4.00 \mathrm{E}-07$ \\
\hline delK & $/ \mathrm{kHz}$ & $-12.7398(13)$ & $-12.7393(13)$ & $1.30 \mathrm{E}-03$ & $5.00 \mathrm{E}-04$ \\
\hline HJ & $/ \mathrm{Hz}$ & $0.0103882(65)$ & $0.0103841(65)$ & $6.50 \mathrm{E}-06$ & 4.10E-06 \\
\hline HJK & $/ \mathrm{Hz}$ & $-0.02516(37)$ & $-0.02512(38)$ & $3.80 \mathrm{E}-03$ & $4.00 \mathrm{E}-05$ \\
\hline HKJ & $/ \mathrm{Hz}$ & $-1.9061(14)$ & $-1.9066(14)$ & $1.40 \mathrm{E}-03$ & $5.00 \mathrm{E}-04$ \\
\hline HK & $/ \mathrm{Hz}$ & $31.6144(98)$ & $31.6134(96)$ & $9.60 \mathrm{E}-03$ & $1.00 \mathrm{E}-03$ \\
\hline hj & $/ \mathrm{Hz}$ & $0.0039706(27)$ & $0.0039703(27)$ & $2.70 \mathrm{E}-03$ & $3.00 \mathrm{E}-07$ \\
\hline hjk & $/ \mathrm{Hz}$ & $0.10155(36)$ & $0.10144(36)$ & $3.60 \mathrm{E}-04$ & $1.10 \mathrm{E}-04$ \\
\hline hk & $/ \mathrm{Hz}$ & $6.497(25)$ & $6.500(25)$ & $2.50 \mathrm{E}-02$ & $3.00 \mathrm{E}-03$ \\
\hline LJ & $/ \mathrm{mHz}$ & $-0.00005246(55)$ & $-0.00005217(55)$ & $5.50 \mathrm{E}-07$ & $2.90 \mathrm{E}-07$ \\
\hline LJJK & $/ \mathrm{mHz}$ & $-0.000816(24)$ & $-0.000814(24)$ & $2.40 \mathrm{E}-05$ & $2.00 \mathrm{E}-06$ \\
\hline LJK & $/ \mathrm{mHz}$ & $-0.1201(10)$ & $-0.1202(10)$ & $1.00 \mathrm{E}-03$ & $1.00 \mathrm{E}-04$ \\
\hline LKKJ & $/ \mathrm{mHz}$ & $0.3979(30)$ & $0.3985(30)$ & $3.00 \mathrm{E}-03$ & $6.00 \mathrm{E}-04$ \\
\hline LK & $/ \mathrm{mHz}$ & $-2.383(14)$ & $-2.383(14)$ & $1.40 \mathrm{E}-02$ & $0.00 \mathrm{E}+00$ \\
\hline $\mathrm{lj}$ & $/ \mathrm{mHz}$ & $-0.00002312(29)$ & $-0.00002306(29)$ & 2.90E-07 & $6.00 \mathrm{E}-08$ \\
\hline ljk & $/ \mathrm{mHz}$ & $-0.000688(23)$ & $-0.000682(23)$ & $2.30 \mathrm{E}-05$ & $6.00 \mathrm{E}-06$ \\
\hline $1 \mathrm{kj}$ & $/ \mathrm{mHz}$ & $-0.0443(16)$ & $-0.0441(16)$ & $1.60 \mathrm{E}-03$ & $2.00 \mathrm{E}-04$ \\
\hline $1 \mathrm{k}$ & $/ \mathrm{mHz}$ & $-4.370(39)$ & $-4.378(38)$ & $3.80 \mathrm{E}-02$ & $8.00 \mathrm{E}-03$ \\
\hline PK & $/ \mu \mathrm{Hz}$ & $0.1428(69)$ & $0.1424(69)$ & $6.90 \mathrm{E}-03$ & $4.00 \mathrm{E}-04$ \\
\hline $\mathrm{pk}$ & $/ \mu \mathrm{Hz}$ & $-0.0893(37)$ & $-0.0883(37)$ & $3.70 \mathrm{E}-03$ & $1.00 \mathrm{E}-03$ \\
\hline $\mathrm{rms}$ & $/ \mathrm{MHz}$ & 0.079835 & 0.079757 & & \\
\hline \# lines & & 5683 & 5798 & & \\
\hline
\end{tabular}

a) The global line list from was displaced with CP-FTmmW line list in the frequency range $260-295 \mathrm{GHz}$

b) The magnitude of the difference between parameters determined from the CP-FTmmW displaced global fit and the unaltered global fit. 

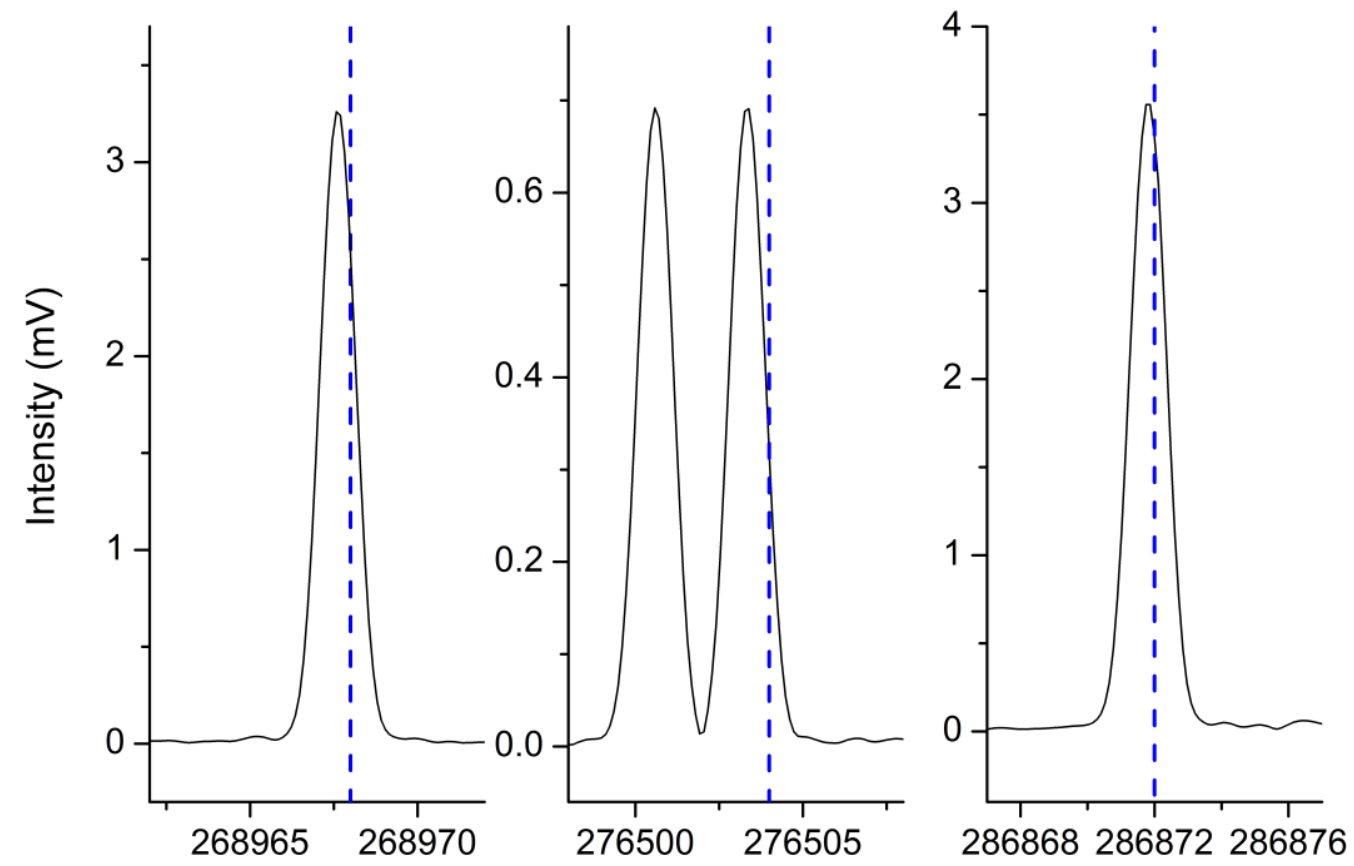

Frequency $(\mathrm{MHz})$

Supplementary Figure 1.1: A 10,000 shot, 2 mTorr spectrum of ethyl cyanide measured on the CP-FTmmW spectrometer (black) in HDR mode. Several spectral lines fall on the $24 \mathrm{MHz}$ segment splices (blue). After correction and up/down sweep averaging the line shape is practically unaffected by the segmenting. 


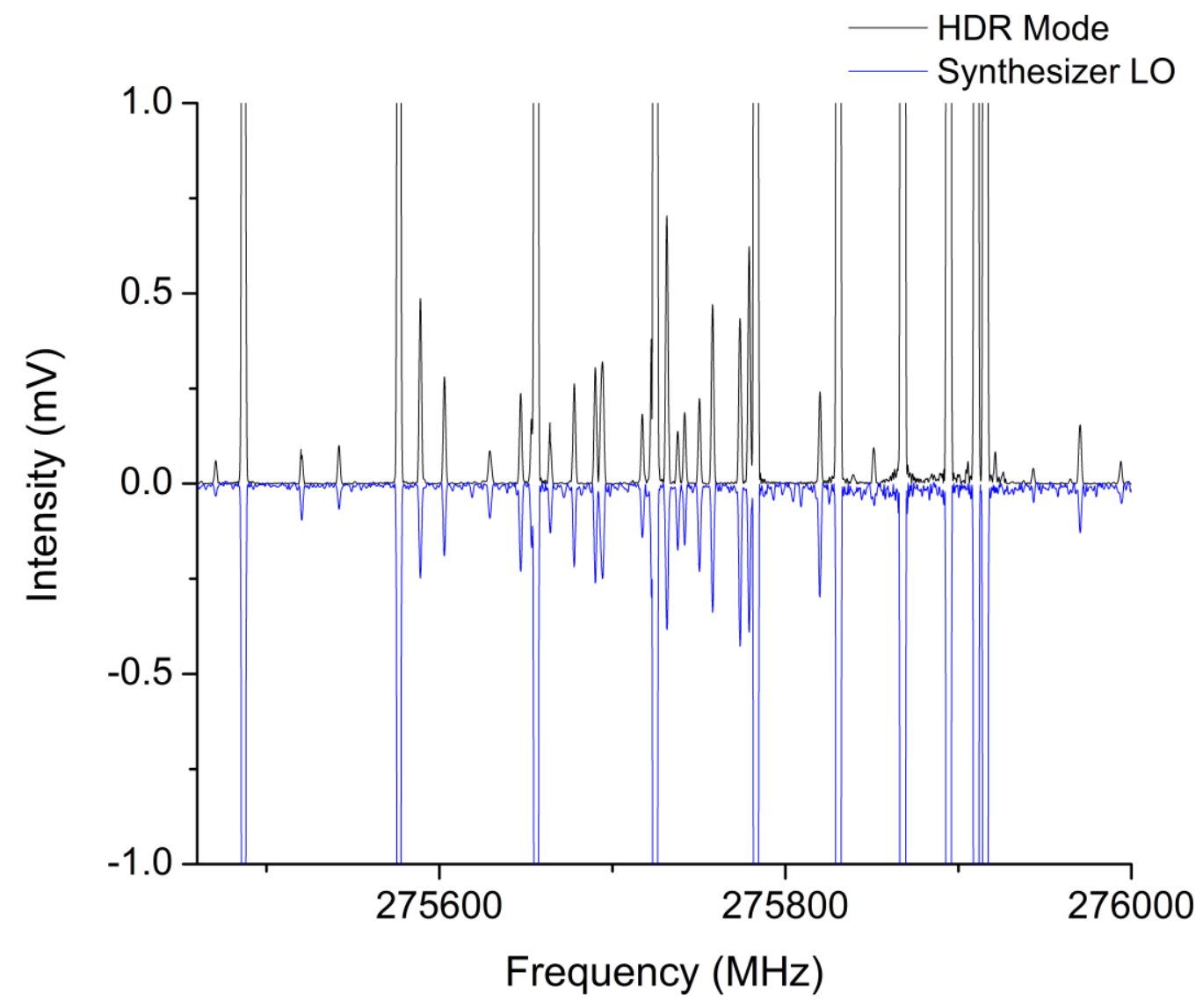

Supplementary Figure 1.2: A 10,000 shot, 1 mTorr spectrum of acetonitrile measured on the CP-FTmmW spectrometer. For the HDR mode (black), the source power was attenuated from $14 \mathrm{mWatts}$ to $1 \mathrm{~mW}$ in order to mitigate receiver saturation. The fast mode spectrum using a continuous wave high purity microwave synthesizer (blue) is scaled up to match the signal height of the HDR spectrum. The signal-to-noise in the HDR spectrum is $350,000: 1$ (70 $\mathrm{mV}$ peak signal). HDR spectral purity exceeds that of a wider band spectrum $(720 \mathrm{MHz})$ generated with a pure LO. 


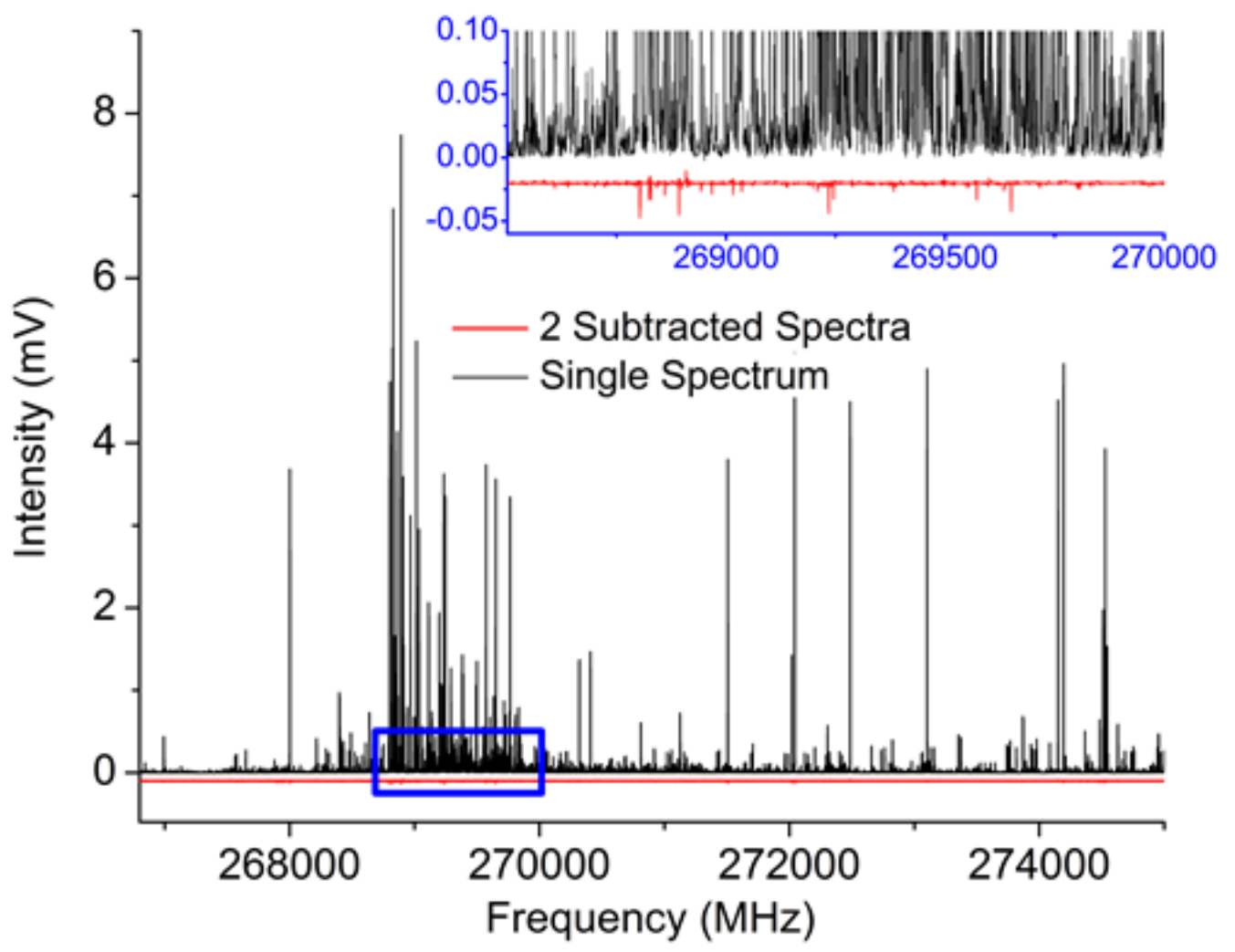

Supplementary Figure 1.3: A 10,000 shot, 2 mTorr spectrum of ethyl cyanide measured on the CP-FTmmW spectrometer (black) in fast mode. The subtraction of two measurements back to back (red) yields a measurement precision better than $0.5 \%$. 


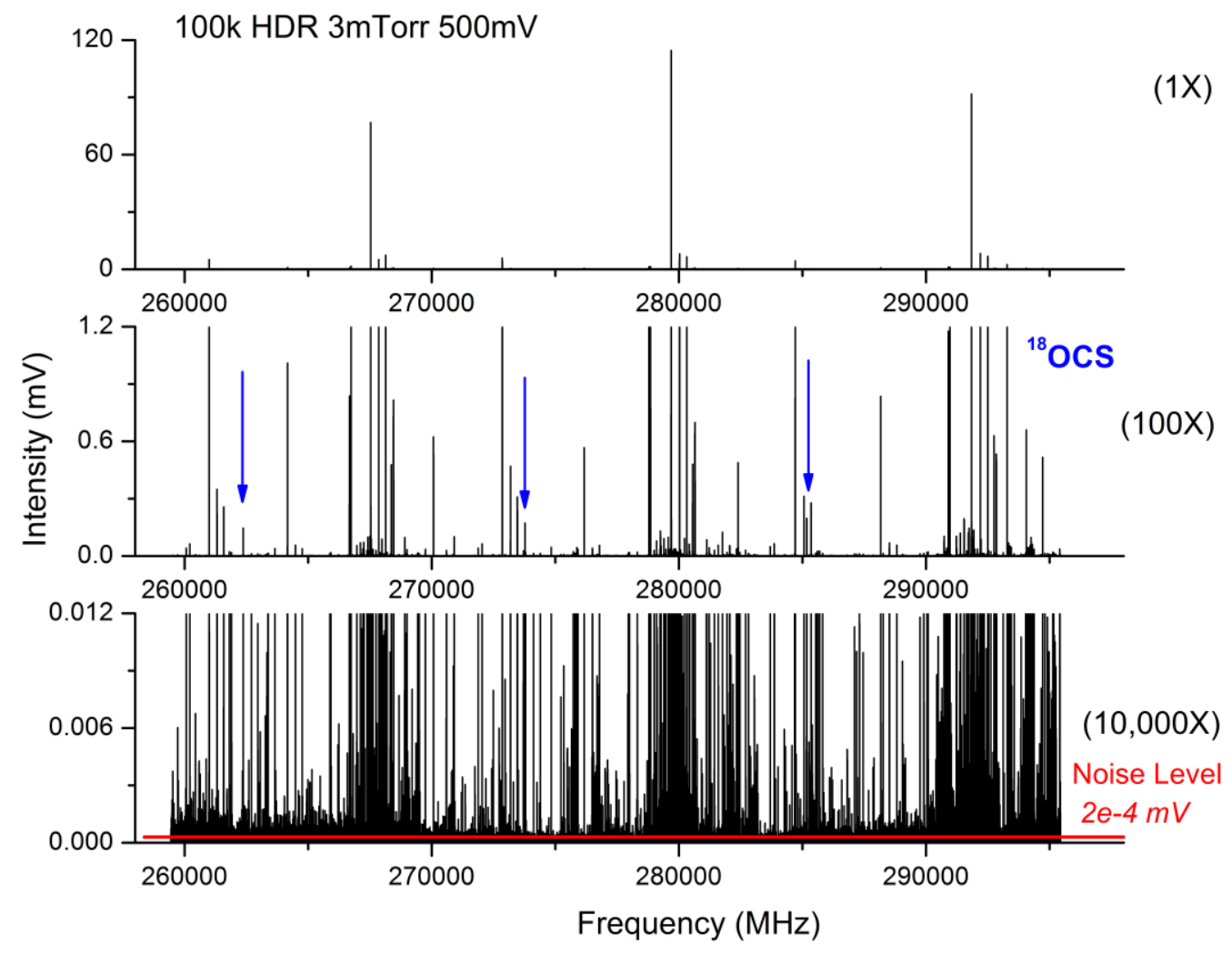

Supplementary Figure 1.4: A 100,000 shot, 3 mTorr spectrum of OCS measured on the CP-FTmmW spectrometer in HDR mode. Even in the OCS spectrum, a 10,000X zoomed in scale of the baseline reveals a well-populated full band spectrum. Most of these lines are due to all of the vibrationally excited modes and isotopologues of OCS as indicated by the repeating patterns in the $100 \mathrm{X}$ zoom, however the spectrometer is sensitive to nTorr amounts of contaminants degassing from regulators, sample transfer lines, and the sample cell wall. The purity specification for the sample is $>97.5 \%$. 


\section{Chapter 2. Analysis Tools}

\section{Advanced gas analysis tools for Fourier transform millimeter spectroscopy}

\section{Introduction}

Historically, the introduction of Fourier transform techniques has been transformative for specific techniques in spectroscopy. [1-3] The same is true for microwave spectroscopy. The sensitivity benefits of Fourier transform microwave (FTMW) rotational spectroscopy have been realized since its introduction in the early 1980s, and soon thereafter became the dominant technique for microwave measurements effectively supplanting microwave absorption spectroscopy. [4-8] However, it never completely displaced all use of direct absorption rotational spectroscopy for several reasons. FTMW is an exception to the typical case that FT techniques are broadband measurements. Although FTMW is highly sensitive, it is a narrow band (high Q) cavity measurement and is not practical for wide-band surveys of the spectrum. Since 2006, chirped-pulse Fourier transform microwave spectroscopy (CP-FTMW) has transformed microwave Fourier transform microwave spectroscopy into a broadband field. [9-15] Both FT-MW and CP-FTMW measurements are predominantly performed on supersonic jet expansions, which cool the sample and shift the rotational population distribution towards lower energy levels probed in the microwave frequency range. Warm, low pressure gases (either in a flow cell or static) are still studied using millimeter/submillimeter-wave direct absorption spectrometers. [16-27] Over the last few years, chirped pulse methods have been extended to higher frequency measurements. [28-31] Recent developments in CP-FT millimeter wave (CP-FTmmW) spectroscopy 
have addressed the challenges of producing high quality, fast, millimeter wave spectra at a practical cost by broadband Fourier transform techniques. [32] The primary enabler is the segmented approach, which is used to maintain low frequency signal digitization by addressing the spectrum in small bandwidth increments without any tradeoff in sensitivity. Segmented CP-FTmmW realizes the full sensitivity gains of chirped-pulse rotational spectroscopy, because it can be performed with practically real-time signal averaging using FPGA equipped PCI digitizers. In addition to significant sensitivity gains, time-resolved, coherent spectroscopy can enhance the analysis in ways that are not well suited for direct absorption methods. The work presented here summarizes a suite of advanced analysis techniques for FTmmW spectroscopy of room temperature static gases.

For full composition analysis, the rotational spectrum can pose a challenge. A typical broadband millimeter-wave spectrum covering at least $30 \mathrm{GHz}$ can have on the order of 100,000 independent data channels which can hold hundreds of signals for just one molecule in a mixture. [28,32-35] Techniques for composition analysis by rotational spectroscopy have historically been driven by the field of astrochemistry, where broadband spectral line surveys of molecular clouds and circumstellar envelopes are used to catalogue the chemical makeup of the interstellar medium. [36-43] Even with the extensive, publicly available libraries of reference line lists, data sets that are decades old still contain a large percentage of unassigned lines. [44-46] For the unassigned parts of the spectrum, the conventional approach to analysis has been to intuit what the chemical composition might be based on the most current chemical modeling and then synthesize the suspect molecular carriers in the laboratory. Once the broadband spectrum is 
acquired in the laboratory it can be compared to astronomy data sets for matches and either an upper limit or an estimated abundance of the molecule can be catalogued by applying standard antennae theory, quantum calculations and radiative transfer models to estimate the strength of the light field (and the column density of the molecule). This approach results in approximately 4 new detections each year across an international effort. At that pace, astrochemists have learned a great deal about the composition of the interstellar medium; however, the broadband spectral data rate of new submillimeter interferometers like the Atacama Large Millimeter/submillimeter Array (ALMA) and the Karl Jansky Very Large Array (JVLA) is exceeding the analysis rate. New approaches to full composition analysis are necessary for both astrochemistry research and in order to make rotational spectroscopy a practical technique for terrestrial analytical applications.

In the case of analytical gas sensing where a specific chemical (or set of chemicals) is being monitored, a common metric for comparison between instruments is the lower limit of detection (LOD). When interpreted properly, the lower limit of detection is the minimum value at which the pure analyte will produce a confidently discernible response for the instrument. When factoring in matrix dependent crosstalk in real samples, the LOD may not be achievable. Analytical results are always a report of the upper limit of the analyte abundance. Consequently, the false positive detection rate is an important characteristic of a particular technique. Because of the high-resolution nature of rotational spectroscopy, it is ideal for statistically low false positive gas sensing. In Fig. 2.1 the high dynamic range, 10 minute, CP-FTmmW spectrum of OCS highlights the complex nature of trace measurements in analytical gas sensing. The OCS ( $\geq 97.5 \%$ 


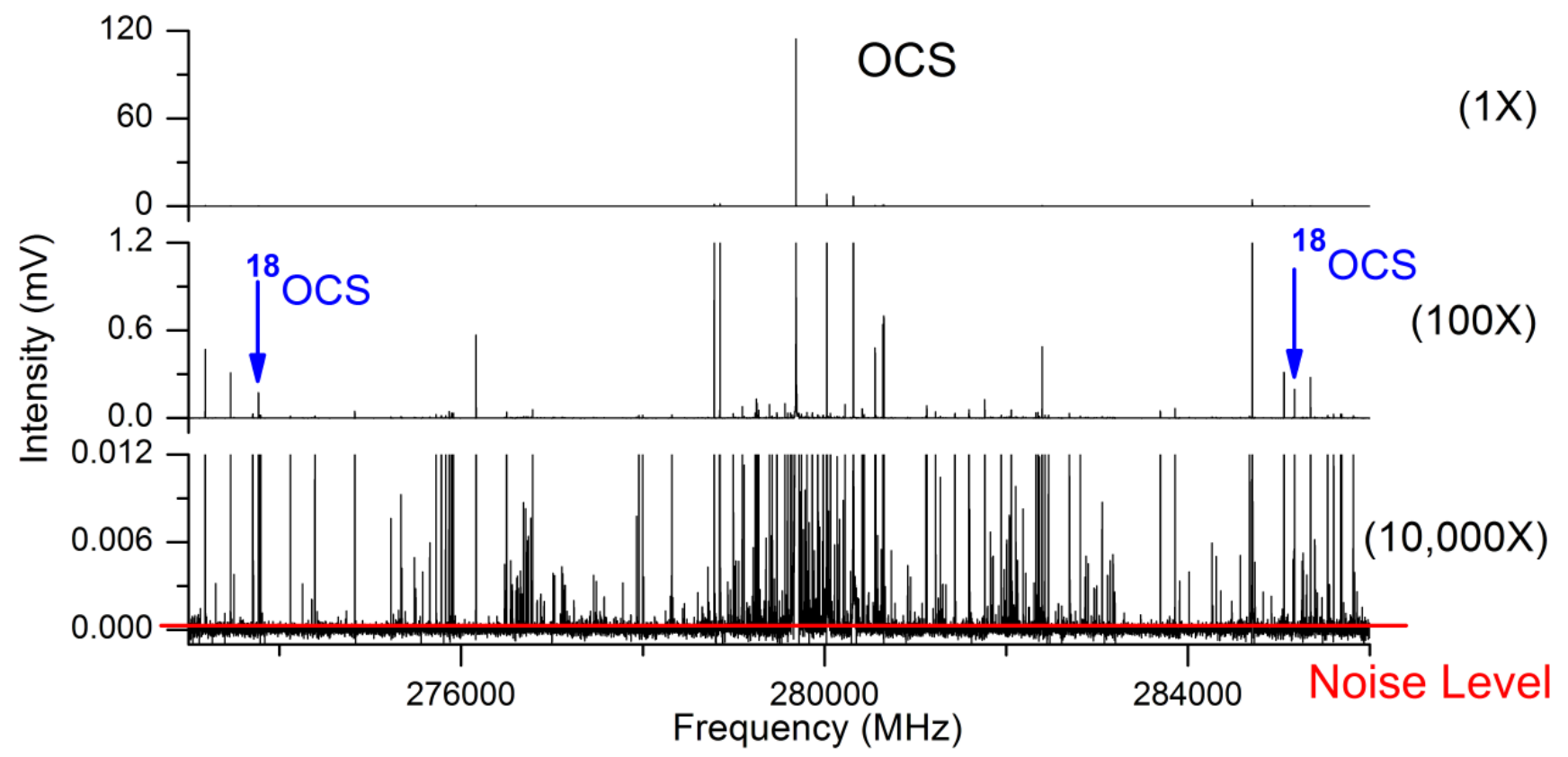

Figure 2.1: A 100,000 shot segmented CP-FTmmW spectrum of OCS at 3mTorr acquired in high dynamic range mode. The spectrum is comprised of contributions from vibrationally excited states, isotopologues, and impurities. 
purity) spectrum represents the most favorable case for a "simple" analysis of a linear rotor through high-resolution millimeter wave spectroscopy. Upon close inspection of the baseline, it is clear that the "pure" spectrum is largely unassigned and would represent a time intensive task to catalogue the full composition. Across nearly 6 orders of magnitude in dynamic range, the spectrum includes contributions from vibrationally excited modes, isotopolgues, sample impurities, and impurities introduced from the spectrometer sample cell. As dense as the OCS spectrum is, the high-resolution nature of rotational spectroscopy leaves empty data channels. However, multi-carbon asymmetric top molecules can exhibit confusion limited spectra at the baseline and limit the dynamic range. For example, recent CP-FTmmW benchmark results have reported the confusion limit at $0.1 \%$ of the strongest for room temperature ethyl cyanide (which is above the true noise level of the measurement). [32] The instrument is capable of a $10^{6}: 1$ dynamic range (or more), but the spectroscopy of the mixture may not be. Stringent tests for line assignments are required for reliable gas sensing by millimeter wave spectroscopy.

The broadband measurement speed of CP-FTmmW (and sensitivity) is just one advance that is necessary to realize the potential of gas analysis by rotational spectroscopy. Full band CP-FTmmW gives the analyst an unbiased view of the entire matrix. Library matching of reference spectra is a straightforward task for assigning most of the spectrum quickly. The arbitrary waveform generator based instrument can also customize a measurement routine for a more sensitive targeted search for specific chemicals. However, for the spectrum that is still left unassigned, time domain spectroscopy has capabilities for speeding the identification of unknowns and for reducing false positive detections. The capabilities are derived from the time resolved 
nature of the coherent measurement, and the reprogrammable light field. This work applies common FT techniques such as $\pi / 2$-pulse optimum coherence excitation, double resonance, signal rephasing (echoes), and molecular free induction decay (FID) analysis in order to enhance the analysis of the spectrum. Since all of the analytical tools described require only single color excitation pulses, the spectrometer design was adapted to include low-cost microwave synthesizers in place of the arbitrary waveform generator and the receiver was modified for low frequency digitization by utilizing a double conversion configuration.

\section{Experimental}

The spectrometer used in this work is the same as described previously [32] but with some adaptations. Light synthesis in the original CP-FTmmW spectrometer is driven by a dual channel, $12 \mathrm{GS} / \mathrm{s}$, 12-bit arbitrary waveform generator (AWG). One channel is used to generate the linear frequency sweeps for broadband excitation (chirps) and the other channel is used to generate a local oscillator (LO) sine wave which drives the heterodyne receiver and restricts the intermediate frequency (IF) detection band to $720 \mathrm{MHz}-1440 \mathrm{MHz}$. The chirped pulses and LO sine waves are generated between 2-4 GHz, are upconverted off of a common $8.8 \mathrm{GHz}$ PDRO to $10.7-12.2 \mathrm{GHz}$ and multiplied in solid-state, Schottky diode active multiplier chains (AMCs). The excitation source is a $24 \mathrm{x}$, high power AMC, with $20-45 \mathrm{~mW}$ of output power across the $260-295 \mathrm{GHz}$ spectrometer band. The receiver is a $12 \mathrm{x}$ AMC combined with a subharmonic mixer, subsequent amplifiers and bandpass filters. All signal generators are locked to a $10 \mathrm{MHz}$ rubidium disciplined quartz oscillator. 
The measurement cycle for each spectral segment is $2 \mu \mathrm{s}$ and includes a short excitation chirp (24 MHz in $100 \mathrm{~ns}$ for high dynamic range mode, $720 \mathrm{MHz}$ in $200 \mathrm{~ns}$ for fast mode) followed by detection time with no excitation light. During detection, the receiver is driven by the LO. The molecular FID is downconverted in the receiver and recorded on an 8-bit, 4GS/s, PCI digitizer equipped with a 32-bit field programmable array (FPGA). The FPGA can accumulate up to $2^{24}$ spectra (about 16 million) with a maximum record length of $512 \mathrm{kpts}$ for real-time signal averaging.

Although the AWG based segmented CP-FTmmW spectrometer can be easily reprogrammed to carry out the multiple measurement modes presented here, it is not required to generate the single frequency square wave excitation pulses used for the analytical tools. In a modification of the spectrometer (details published elsewhere), the AWG is replaced with a low cost dual channel $137.5-4400 \mathrm{MHz}$ frequency synthesizer equipped with a pin-diode switches on each channel for modulating the excitation pulse output. The dual channel synthesizer enables double pulse excitation. A separate microwave synthesizer of the same type is used to generate the LO. Since only one narrow band frequency range $(5 \mathrm{MHz})$ is measured at a time, the IF can be maintained below $100 \mathrm{MHz}$ and relieve the sampling requirements of the digitizer. In the narrow band spectra, deep averages of weak molecular lines displayed a continuum of emission which degraded the dynamic range. The effect was attributed to the low IF frequency operation of the subharmonic mixer and corrected by modifying the receiver to work at higher IF (approx. $4 \mathrm{GHz}$ ) and include a second down conversion step. Channel two of the LO microwave synthesizer is used for the second stage LO input of the receiver. In the double conversion receiver, there is an added benefit of compression of the excitation 
pulse by the time it reaches the final IF amplifier, which reduces the saturation and recovery effects.

The sample cell is a $65 \mathrm{~cm}$ long stainless $3.8 \mathrm{~cm}$ diameter steel tube (approximately 1L of volume) enclosed with PTFE focal lenses and equipped with a sampling port. Attached is a turbo dry scroll pump combo (Agilent 96982147) and the cell pressure is monitored with a MKS Baratron Capacitance Manometer (50mTorr) pressure gauge. All chemical samples used in this work were obtained from Aldrich Chemical and used without further purification.

\section{Results}

\section{Concepts for Advanced Analysis Tools: More Information for Unassigned Lines}

Until recently, high frequency $(>100 \mathrm{GHz}$ ) rotational spectroscopy had been exclusively carried out using direct absorption which has some drawbacks compared to coherent, chirped-pulse spectroscopy. For direct absorption spectroscopy, the spectrum is constructed point by point as the light source (millimeter or submillimeter) is incrementally tuned across a broad frequency band and the power transmitted through the molecular sample is measured. When the frequency of the light source comes into resonance with a molecular rotational transition, the radiation is absorbed with a corresponding drop in transmitted power. Tuning accuracy for millimeter/submillimeter light sources is many times better than an absorption line width, and the light source stability is sufficiently high for high-resolution sampling of the spectral line. Due to the fact that detection takes place against the bright background excitation light, the baseline noise level is influenced by the spectral purity of the light source and etalon effects, or 
standing waves. Spline reprocessing can restore the flat baseline, but it can degrade the dynamic range of the measurement because weak signals can be distorted in the process. [32] The "stepped" nature of the measurement limits the broadband scanning speed, where each of the greater than 100,000 measurements is several times a typical transition dephasing time. It also concentrates all of the source power into a bright, single frequency element requiring attenuation in order to avoid saturation of molecular transitions and power broadening at the low pressure sampling conditions. Since the saturation level is molecule specific, the attenuation level cannot be universally optimized. [16]

Efficient use of the millimeter wave source power is an important advantage for chirped pulse spectroscopy. Using short, linear frequency sweeps of excitation light, the AMC power is distributed across a wide frequency band so that all of the power can be utilized without saturation. As a result, high power AMCs $(45 \mathrm{~mW})$ can be incorporated to directly improve the sensitivity of the spectrometer. The simplest measurement scheme involves coherently driving molecular transitions across the entire band of the spectrometer with one chirped pulse. [28] In contrast to absorption, molecular emission is detected against a zero background. Once the excitation pulse is complete, the coherent, decaying electric field of pure molecular emission is traced out on a high-speed digitizer. The entire frequency axis is constructed at once in the fast Fourier transform just as in time domain FTMW spectroscopy, but with a much broader bandwidth. Unlike absorption spectroscopy, the zero background circumvents any need for baseline spline processing. There are practical design advantages to segmenting the spectrum into smaller bandwidth sections and concatenating the spectrum piece by piece without any 
tradeoff in sensitivity. [32] By maintaining a low bandwidth digitization across the spectrometer band, real-time signal processing can accomplish a near $100 \%$ duty cycle. In the CP-FTmmW spectrometer, all the power and all the time goes into measurement and sensitivity improvement resulting in a 25,000 fold enhancement of broadband sensitivity compared to modern direct absorption spectrometers. [32, 48] In addition to speed and spectral quality, the time domain nature of CP-FTmmW enables more control and yields more spectral information.

\section{A. Coherent Excitation}

One key distinction for coherent, time domain spectroscopy is that the duration of the excitation pulse can be controlled to directly influence the magnitude of the coherence. Approximating a rotational transition as a two level system, the coherence can be illustrated in a unit Bloch sphere, where the time evolution of the state is represented by a vector oscillating on the $\mathrm{x}-\mathrm{y}$ plane according to the real and imaginary components of the phase and projecting on the $\mathrm{z}$-axis according to the rotational upper $(+\mathrm{z})$ and lower state (-z). When coupled to a coherent light field, the transition probability between the upper and lower states oscillates with time according to the Rabi frequency $\left(\omega_{R}\right)$, represented in the unit Bloch sphere as the vector projection on the z-axis.

$$
\omega_{R}=\frac{\mu_{10} E}{\hbar}
$$

When the driving electric field is on resonance with the transition, the Rabi frequency is dependent upon the electric field strength $(E)$ and the transition dipole moment $\left(\mu_{10}\right)$. The Rabi oscillation can be traced out indirectly by monitoring the signal intensity for a particular molecular transition as a function of the excitation pulse duration $\left(t_{p}\right)$, referred 
to herein as a nutation curve. The time domain signal $(S)$ oscillates and decays according to equation 2.2. Relaxation is included in the expression through an exponential dampening factor with lifetime $(\tau)$.

$$
S \propto e^{\frac{-t_{p}}{\tau}}\left|\sin \left(\omega_{R} t_{p}\right)\right|
$$

The $\pi / 2$ angle corresponds to the maximum magnitude of the coherence with the Bloch vector completely oriented along the $x-y$ plane. The aim for chirped pulse spectroscopy is to excite in the weak pulse limit where the Rabi angle is well below $\pi / 2$. However, for single frequency excitation pulses, the transform limited bandwidth is sufficiently small to concentrate the source power such that typical excitation pulse durations from $100-$ 200 ns can lead to $\pi / 2$ conditions. For monitoring single transitions, this technique can be used to improve sensitivity. In contrast to the weak pulse limit, the line strength scales with the dipole moment, $\mu$, as opposed to $\mu^{2}$ because the Rabi effect of $\mu$ has been removed. A nutation curve can be measured for each species and each molecular transition of interest in order to ensure optimum signal strength (Fig. 2.2).

In addition to improving sensitivity, the nutation curve can be leveraged as additional information for aiding in the assignment of an unknown spectral transition. The Rabi relationship sets up the dependence between the electric field strength and the transition dipole moment. So long as one is known, the other can be determined. This has been used in the past to verify the strength of the electric field in a spectrometer [31], but it can be used conversely when the strength of the electric field is known in order to estimate a dipole moment. Since the transition dipole moment includes an overlap integral, the challenge is complicated by (and enriched by) the transition type (a, b, or c) which can be used to determine the dipole projection on the three principal axes. The 


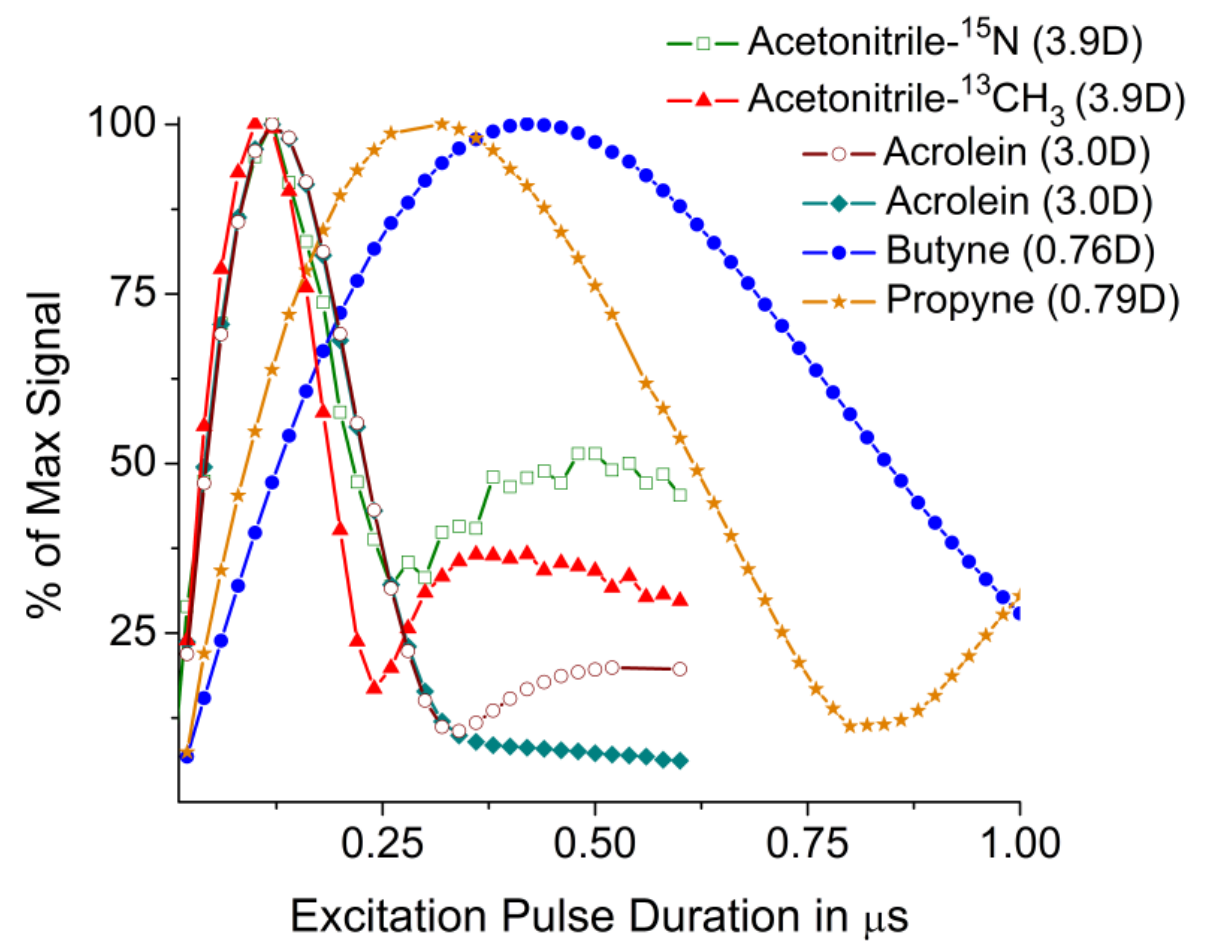

Figure 2.2: Nutation curves for several different molecular transitions. The optimum signal is achieved for a $\pi / 2$-pulse duration, where the coherence is strongest and is dependent upon the transition dipole moment and the electric field strength. The molecular transitions include: Acetonitrile $-{ }^{15} \mathrm{~N} 16_{(2)} \rightarrow 15_{(2)}$, Acetonitrile $-{ }^{13} \mathrm{CH}_{3} 16_{(2)}$ $\rightarrow 15_{(2)}$, Acrolein $32_{2,31} \rightarrow 31_{2,30}$, Acrolein $32_{1,32} \rightarrow 31_{1,31}$, Butyne $32_{9,24} \rightarrow 31_{9,23}$, Propyne $16_{(2)} \rightarrow 15_{(2)}$ 
information can be useful as supplementary information that must be consistent with an initial assignment "guess." Once a guess is established (through analytical tools described in this work) and there is confident dipole moment estimation, the assignment can be accompanied with an estimated abundance. Since the advanced analysis techniques for this work all employ single color, transform limited excitation, the nutation curve is used as a fundamental measurement to enable ancillary information about the spectrum.

\section{B. Free Induction Decay}

The molecular FID can be modeled as a sinusoid, which decays from its initial amplitude according to [7]

$$
E(t)=A \sin \left(\omega\left(t-t_{0}\right)+\phi\right) \exp \left(\frac{-\left(t-t_{0}\right)}{T_{1}}\right) \exp \left(\frac{-\left(t-t_{0}\right)^{2}}{4 s^{2}}\right)
$$

Where the sinusoid is defined by amplitude $(A)$, frequency $(\omega)$, time zero $\left(t_{0}\right)$, and phase $(\phi)$. There are a few effects which cause the signal to decay. The first is Doppler dephasing defined by the decay constant $(s)$. For a static gas measurement (as opposed to a molecular beam), at a particular temperature there is an average kinetic translational energy with random distribution in space. Molecular emission will be coherent with some inhomogeneous width to the frequency distribution due to the Doppler effect. Since the mean velocity of an ensemble of particles is mass dependent (to conserve kinetic energy, $\left.E_{k}=m v^{2}\right)$, the Doppler decay of the coherence will also be mass dependent. The Doppler line width $\left(\Delta v_{D}\right)$ can be calculated according to:

$$
\Delta v_{D}=\frac{\omega}{c} \sqrt{\frac{2 k T \ln (2)}{M}}
$$

Where $c$ is the speed of light, $k$ is the Boltzmann constant, $(T)$ is temperature and $(M)$ is the molecular mass. The other effects contributing to signal decay are interactions, such 
as molecular collisions, which can cause either a loss in phase $\left(\mathrm{T}_{2}\right)$ or a transition to another state (thermal equilibration $\mathrm{T}_{1}$ ). For rotational spectroscopy the probabilities of these two effects are expected to be approximately equal $\left(\mathrm{T}_{1} \approx \mathrm{T}_{2}\right)$. [47]

The Doppler $\exp \left(-\mathrm{t}^{2}\right)$ decay contributes a Gaussian line shape which has a relatively sharp fall to the baseline in the frequency domain and the collisional $\exp (-\mathrm{t})$ decay contributes a Lorentzian line shape with long tails. In order to avoid the deleterious effects to dynamic range of the Lorentzian broadening, absorption spectroscopy is carried out at sufficiently low pressure to ensure Doppler limited spectra. Longer-lived coherence, corresponds to a narrowing of the spectral line by Fourier transform, however signal processing by zero padding and windowing the decay profile constrains the line shape up to higher pressures, suppressing Lorentzian wings in the frequency spectrum. The signal processing capabilities are inherent advantages that allow CP-FTmmW spectrometers to operate at higher total pressures and enhance the concentration detection limits over absorption spectrometers. When the pressure is sufficiently high to exceed the Doppler limit, the absorption lineshape includes both Lorentzian and Gaussian contributions that are approximated by a Voigt profile. An independent measurement of the Gaussian contribution is possible through time domain spectroscopy without knowing the molecular carrier a priori and offers an alternative to conventional line shape analysis. [48]

\section{Signal Echoes}

Since the Doppler dephasing will dephase according to a set frequency distribution starting at time $\mathrm{t}_{0}$, so long as a collision has not reset the phase (or changed 
the state), the Bloch vector can be inverted with a $180^{\circ}$ pulse to, in effect, reverse the time axis and send the dephased emission back to $t_{0}$. This phenomenon of rephasing the inhomogeneous decay effects has been exploited in NMR, referred to as Hahn echoes. [49] It is common to the optical regime and has been extended from microwave spectroscopy to millimeter spectroscopy as well. [50-53] If there were no collisions, then the echoes would constructively interfere to the same amplitude as the start of the original FID. However, in the presence of collision, the echo amplitude will have decayed in time according to $\exp \left(-\mathrm{t} / \mathrm{T}_{1}\right)$. The $\operatorname{pump}(\pi / 2)$-probe $(\pi)$ delay can be incrementally increased in order to trace the echo amplitude as a function of time and determine the $\mathrm{T}_{1}$ decay constant independently of the Doppler constant (see Fig. 2.3). The Doppler decay constant can then be fit to the FID decay in order to determine a mass.

\section{Mass Estimation}

The mass measurement mode utilizes a single frequency excitation pulse tuned to the center frequency of an unassigned spectral line (with duration defined by the nutation curve) followed by $98 \mu$ s of off time in order to allow sufficient collisional decay of the FID so that consecutive excitation pulses will not generate interfering echoes. In contrast to the $720-1440 \mathrm{MHz}$ IF range used in the broadband HDR mode, the IF is chosen to be less than $100 \mathrm{MHz}$ so that the FPGA accumulator in the digitizer can accommodate $2 \mathrm{~ms}$ of data at a lower sampling rate $(250 \mathrm{MS} / \mathrm{s})$. Ten, $100 \mu$ s experiments are accumulated in real time so that many independent measurements of the mass can be performed. 


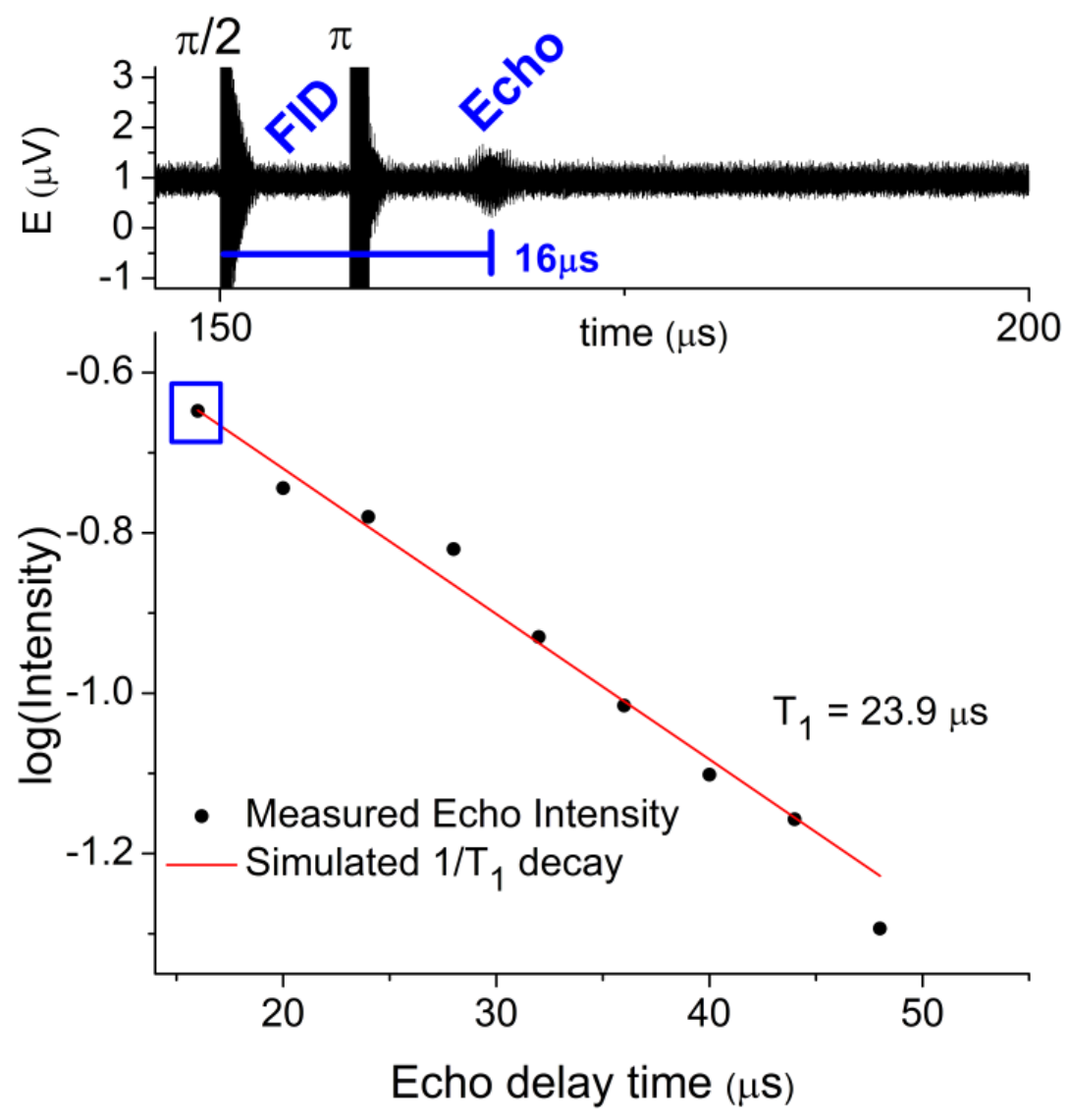

Figure 2.3: The $\mathrm{T}_{1}$ decay constant measured by the Hahn echo decay for the $\mathrm{OC}^{33} \mathrm{~S}$ $\mathrm{J}=23 \rightarrow \mathrm{J}=22$ transition, at $1 \mathrm{mTorr}$ total pressure and 10,000 shots. The time trace (top) shows the first $\pi / 2$ excitation pulse and a delayed $\pi$ excitation pulse with amplitudes off the $y$-axis. An echo appears in time at twice the delay of the second excitation pulse and reduced amplitude according to the $\mathrm{T}_{1}$ decay constant (bottom). 
The 6 parameter FID model (eq. 2.3) is matched to the experimental time trace through a least squares optimization. Four parameters define the sine wave $\left(\mathrm{t}_{0}\right.$, amplitude, phase, and frequency) and two parameters define the decay rate (s and $\left.\mathrm{T}_{1}\right)$. For a $1.8 \mu \mathrm{s}$ FID, the number of data points at $4 \mathrm{GS} / \mathrm{s}$ is 3600 points which provides a better defined optimization than line shape analysis through the frequency spectrum where a spectral line might be defined by only $10-20$ points. However, floating the six parameters for the least squares optimization is still problematic because there is a strong correlation between the two decay rates, the amplitude, and $t_{0}$ (the FID start time). The frequency can be independently determined from the line center in the Fourier transform and fixed for the least squares optimization. As described above, the collisional decay rate $\left(\mathrm{T}_{1}\right)$ can be independently determined from measurement of the echo decay profile leaving $t_{0}$, amplitude, phase, and the Doppler decay rate undetermined. In principle, the phase can be determined from the excitation pulse, but in this case the phase information is only used to define the sinusoid and is not used in further analysis.

Fixing a $\mathrm{t}_{0}$ will result in better than $1 \%$ precision of the Doppler decay constant across multiple FIDs, but it cannot be randomly chosen. The choice of $t_{0}$ can affect mass estimation by over $10 \%$. The best $t_{0}$ is chosen by floating the three undetermined parameters (amplitude, phase, and the Doppler decay rate) across a range of fixed values for $t_{0}$. The minimum residual variance versus $t_{0}$ points to the most confident determination of $t_{0}$ so that it can be fixed in the final fit where the Doppler decay constant, the amplitude, and the phase of the sinusoid are floated parameters.

Early results indicated a strong dependence of the mass determination on the choice of gate which defines where the experimental data trace starts for the FID model. 
As the gate start varies over a few hundred nanoseconds, the resulting mass determination oscillates about an average value. In order to determine the mass with high confidence, the mass result is an average of many independent optimizations. For each FID, the starting time for measurement gate varies in $10 \mathrm{~ns}$ increments across $200 \mathrm{~ns}$ and the average mass is reported. The analysis is performed across 10 FIDs for a total of 200 optimizations. The fitting results illustrated in Fig. 2.4 can be acquired in 30 seconds. A collisional decay constant of $23.9 \mu \mathrm{s}$ for this transition of $\mathrm{OC}^{33} \mathrm{~S}$ is consistent with previous millimeter/submillimeter OCS collisional broadening studies which indicate a collisional decay constant of $26.2 \mu$ s. [54]

The ambient temperature also influences the Doppler decay rate, and was fixed to a value of $298 \mathrm{~K}$. For weak lines, nearby transitions and spurs can also contribute to the decay profile. Narrow band digital filtering can help remove these effects and increase the dynamic range of performance for the mass analysis. First, the FID is Fourier transformed and a first order Bessel window with $30 \mathrm{MHz}$ of bandwidth is applied to the spectrum. Then, an inverse transform is applied to recover the filtered FID. In general, mass results were within $3 \%$ of the actual value across a range of molecules covering mass 32 amu to 68 amu (Fig. 2.5 and Table 2.1). OCS isotopologues $\mathrm{OC}^{34} \mathrm{~S}, \mathrm{O}^{13} \mathrm{CS}$, $\mathrm{OC}^{33} \mathrm{~S}$, and ${ }^{18} \mathrm{OCS}$ were used to benchmark a mass resolution of approximately $1 \mathrm{amu}$. Varying the fixed temperature in the fit by $5 \mathrm{~K}$ can cause a $3 \%$ variation in the determined mass. 


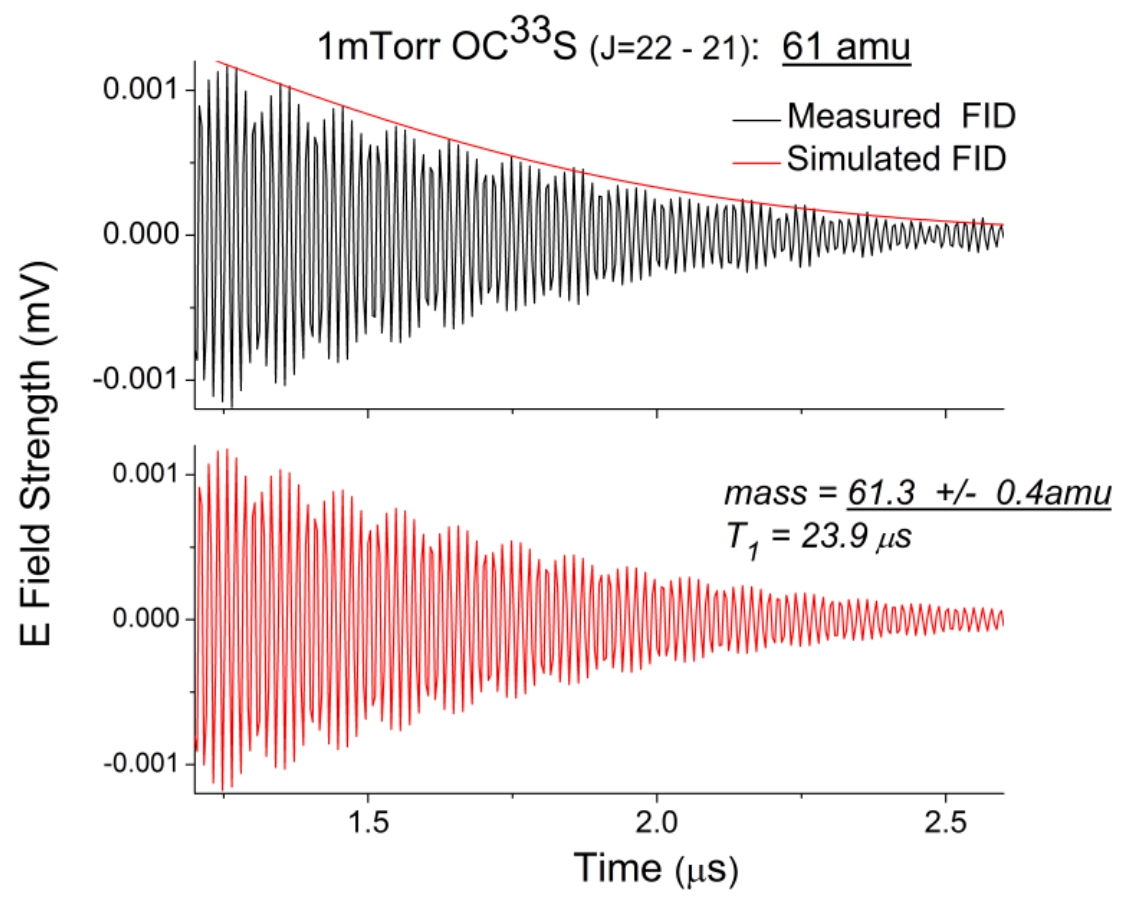

Figure 2.4: The least squares optimization and FID modeling results for mass determination of $\mathrm{OC}^{33} \mathrm{~S}$ (mass $61 \mathrm{amu}$ ) using the $\mathrm{J}=22 \rightarrow 21$ transition measured at $1 \mathrm{mTorr}$. 


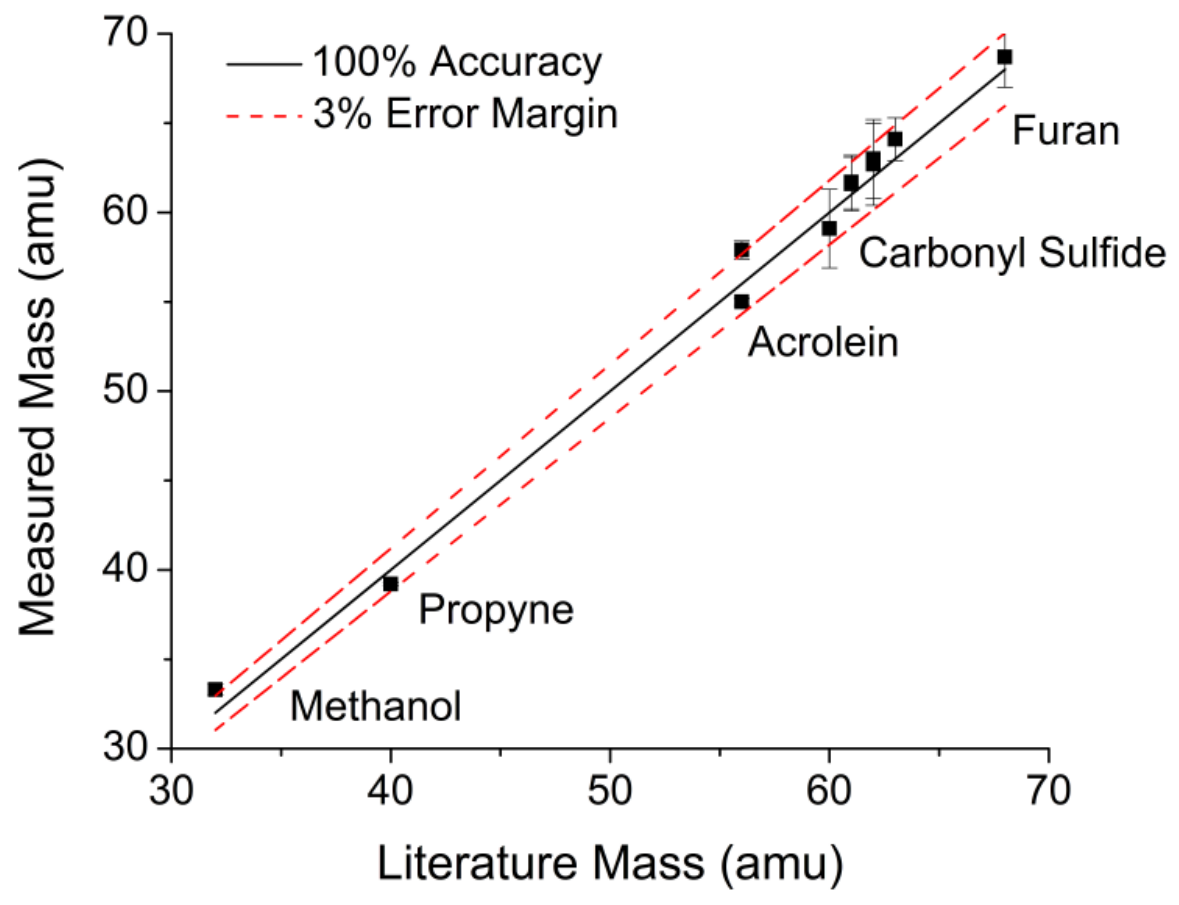

Figure 2.5: The mass results for FID modeling across transitions of several molecules. Four isotopologues of OCS were measured for evaluating $1 \mathrm{amu}$ resolution. 


\begin{tabular}{lcccc}
\multicolumn{5}{l}{ Table 2.1: Molecule Mass by FID Decay Modeling } \\
Molecule & $\begin{array}{c}\text { Literature } \\
\text { Mass (amu) }\end{array}$ & $\begin{array}{c}\text { Measured Mass } \\
(\mathrm{amu})\end{array}$ & $\begin{array}{c}\text { Uncertainty } \\
(\mathrm{amu})\end{array}$ & $\begin{array}{c}\text { Fractional } \\
\text { Error }\end{array}$ \\
\hline Methanol & 32.03 & 33.3 & 0.3 & 0.04 \\
Propyne & 40.03 & 39.2 & 0.1 & 0.02 \\
Acrolein & 56.03 & 57.9 & 0.5 & 0.03 \\
Acrolein & 56.03 & 55 & 0.2 & 0.02 \\
Carbonyl Sulfide-main & 59.97 & 59.1 & 2.2 & 0.01 \\
Carbonyl Sulfide- ${ }^{13} \mathrm{C}$ & 60.97 & 61.7 & 1.5 & 0.01 \\
Carbonyl Sulfide- ${ }^{33} \mathrm{~S}$ & 60.97 & 61.6 & 1.5 & 0.01 \\
Carbonyl Sulfide- $-{ }^{34} \mathrm{~S}$ & 61.96 & 62.7 & 2.3 & 0.01 \\
Carbonyl Sulfide- ${ }^{18} \mathrm{O}$ & 61.97 & 63.0 & 2.2 & 0.02 \\
Carbonyl Sulfide- ${ }^{13} \mathrm{C}^{34} \mathrm{~S}$ & 62.96 & 64.1 & 1.2 & 0.02 \\
Furan & 68.03 & 68.7 & 1.7 & 0.01 \\
\hline
\end{tabular}




\section{E. Double Resonance (two color analysis)}

Another key distinction for time domain spectroscopy is that, in contrast to direct absorption spectroscopy, excitation and relaxation are time separated events. Furthermore, the excitation time is sufficiently short compared to the relaxation dynamics such that multiple excitation pulses can be used in sequence before the FID emission. Both principles are used to demonstrate double resonance identity verification. In this mode, the identity of a molecular carrier in the spectrum can be verified by perturbing the local population of rotational energy levels with a pump pulse and modulating a probe signal. The modulation is a stringent test which confirms the identity of the assigned molecular transition by confirming the presence of its unique energy manifold. Coincidental overlaps would result in zero double resonance modulation. By absorption spectroscopy, this could only be accomplished using a second excitation source, thereby complicating the instrument, increasing the cost, and limiting the interaction region. [55] Since the pump pulse and probe pulse are time separated in time domain spectroscopy, the double resonance effect takes place across the entire beam path.

Double resonance identity verification requires the existence of two connected rotational transitions in the bandwidth of the spectrometer. A simple case is illustrated in Fig. 2.6. A nutation curve for the pump transition is measured in order to determine the $\pi$ condition for population inversion. The $\pi$ pulse for the pump transition enhances the population in the upper state thereby depleting the population of the lower state (by $\Delta$ in Fig. 2.6). The pump transition is chosen such that it shares an energy level with the probe transition. For example, the depleted lower state of the pump transition is the upper state of the connected "probe" transition. After the pump pulse, an increased population 


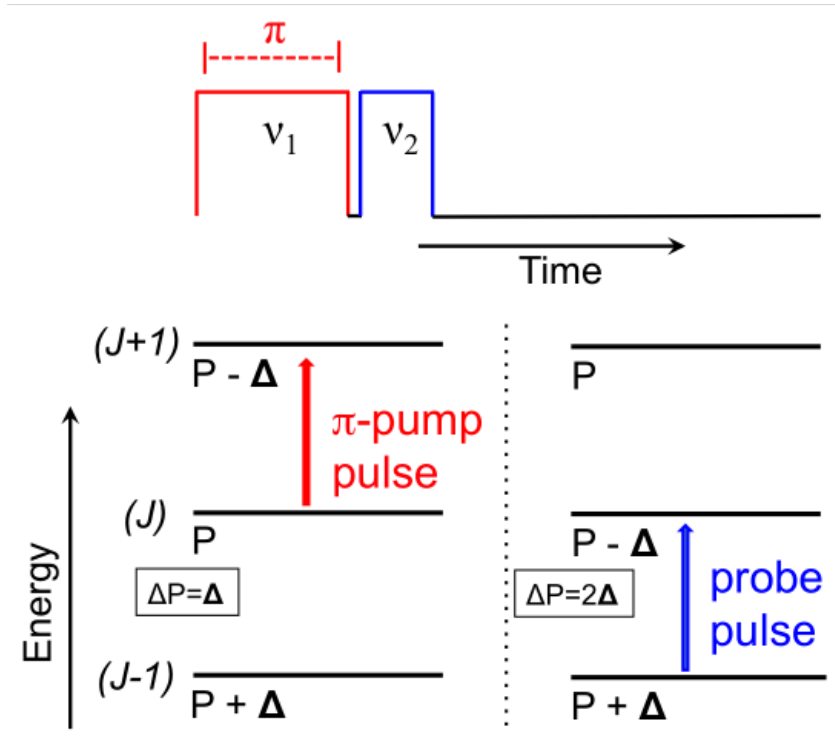

Figure 2.6: Two color, double resonance excitation scheme. First a $\pi$ pulse (red) is applied to invert the population in a connected transition, and then a probe pulse creates a coherence for the probe transition (blue). In principle, the probe signal can be enhanced up to a factor of two due to the higher population difference set up by the pump pulse. 
difference is induced for the probe transition directly influencing a stronger signal coherence. The signal modulation of the probe transition is the double resonance modulation effect.

The energy manifold in Fig. 2.6 resembles that of a simple linear rotor like carbonyl sulfide (OCS). Carbonyl sulfide is an ideal molecule for benchmarking the double resonance performance because of the simple rotational energy level manifold and because the rotational constant (B) is small enough to give rise to three transitions in the spectrometer band. Furthermore, the naturally occurring isotopologues of OCS simulate low concentration conditions, and have become the typical measurement for benchmarking the sensitivity limits of new spectrometers. The value of double resonance verification is greatest in situations where line overlap probability is high, for example, in dense, high dynamic range mixtures. Since the rare isotopologues of OCS have been measured elsewhere in the $260-295 \mathrm{GHz}$ band [56], they are utilized here to verify the performance of the double resonance effect for weak emission using the FPGA equipped, high-speed PCI digitizer. The frequency accuracy of the spectrometer which incorporates microwave synthesizers in place of the AWG is also evaluated. Across 30 transitions, there is a $40 \mathrm{kHz}$ rms center frequency accuracy upon comparison to the measured OCS isotopologue frequencies. [57]

The selectivity of the pump pulse is illustrated in Fig. 2.7 with a double resonance detuning curve for ${ }^{18} \mathrm{O}^{13} \mathrm{CS}$. The maximum modulation of the probe transition $(\mathrm{J}=24 \leftarrow 23)$ will occur as the pump pulse is tuned through resonance with the pump transition $(\mathrm{J}=25 \leftarrow 24)$. A modulation of $60 \%$ can be achieved for a clearly discernible 


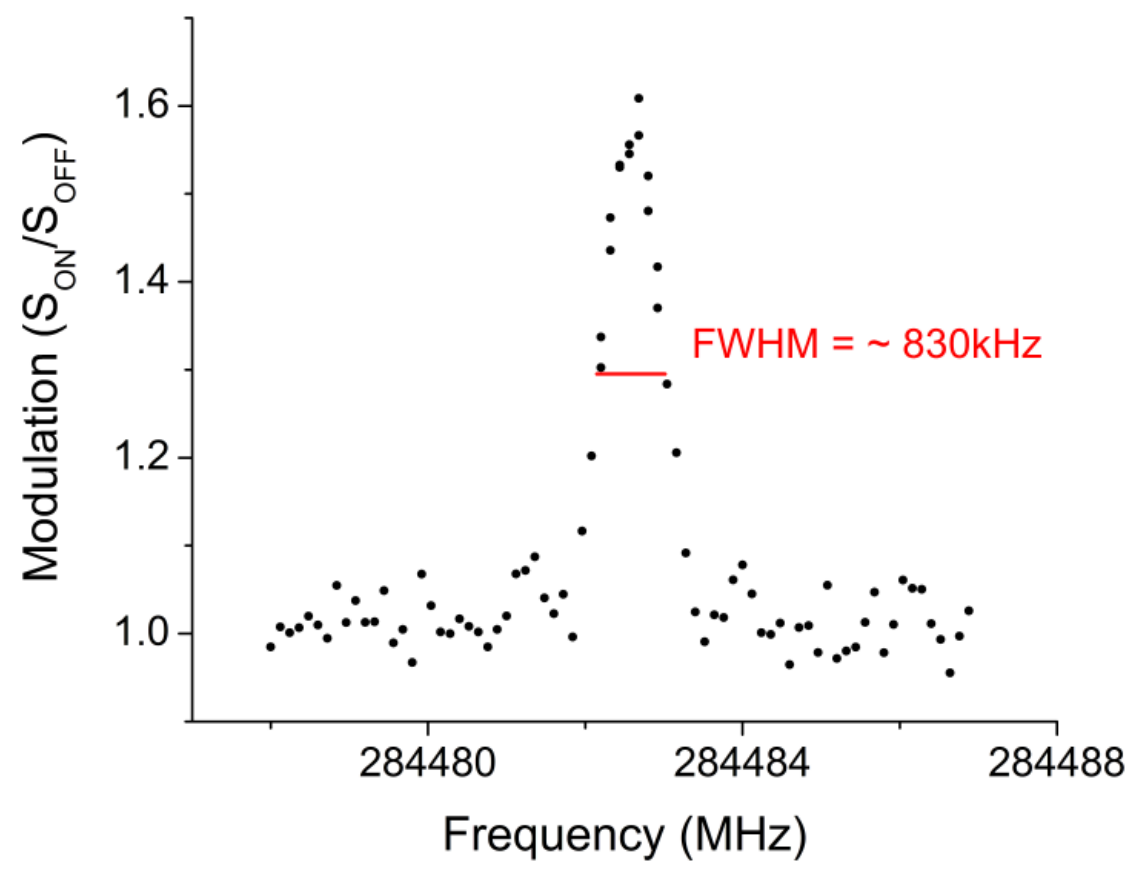

Figure 2.7: The selectivity of the pump pulse illustrated by the double resonance detuning curve for ${ }^{18} \mathrm{O}^{13} \mathrm{CS}$. The pump transition is $\mathrm{J}=25 \rightarrow 24$; the probe transition is $\mathrm{J}=24 \rightarrow 23$. An $80 \%$ modulation can be achieved with resonant tuning. 
double resonance effect. With a duration of approximately $1 \mu \mathrm{s}$, the transform limited power envelope of the pump pulse is sufficiently narrow (1MHz FWHM compared to the typical 2.25 MHz FWHM line width in CP-FTmmW) to selectively modulate the probe transition in a narrow bandwidth of approximately $800 \mathrm{kHz}$. Once the selectivity of the pump pulse was established, the double resonance identity verification mode was applied for the two lowest abundant OCS isotopologues: ${ }^{18} \mathrm{O}^{13} \mathrm{C}^{34} \mathrm{~S}\left(1\right.$ in $10^{6}$ abundance relative to main OCS isotopologue), and ${ }^{18} \mathrm{OC}^{36} \mathrm{~S}$ (1 in $3.4 \times 10^{6}$ relative abundance). Fig. 2.8 illustrates a double resonance modulation of $60 \%$ on the ${ }^{18} \mathrm{OC}^{36} \mathrm{~S} \mathrm{~J}=26 \rightarrow 25$ transition and a detection level of $160 \mathrm{fmol}$ (300 ppb in the sample). In Fig. 2.9, the same measurement was performed on of the $\mathrm{J}=25 \rightarrow 24{ }^{18} \mathrm{O}^{13} \mathrm{C}^{34} \mathrm{~S}$ transition. Within the $5 \mathrm{MHz}$ excitation bandwidth there is a neighboring signal which is unaffected by the double resonance pump pulse, consistent with the high selectivity of the technique.

The application of double resonance described so far only uses single color excitation pulses to verify the identity that has already been assigned to the probe transition. In principle, a full 2D double resonance approach can be applied to the whole spectrum to detect all connected transitions in the spectrum. Rather than extract unassigned lines, the $2 \mathrm{D}$ double resonance mode could be used to extract unknown spectra. The speed of the segmented CP-FTmmW spectrometer can be leveraged to pair a single color $\pi$-pump pulse with chirped pulses that cover the entire band of the spectrometer so that all modulated signals from the pump pulse can be detected. Depending on the sensitivity level required, it could be performed in as short of a time as $100 \mu \mathrm{s}$ using the spectrometer fast mode. Considering a half linewidth of $625 \mathrm{kHz}$, approximately 50,000 pump colors would cover the entire spectrum meaning a complete 


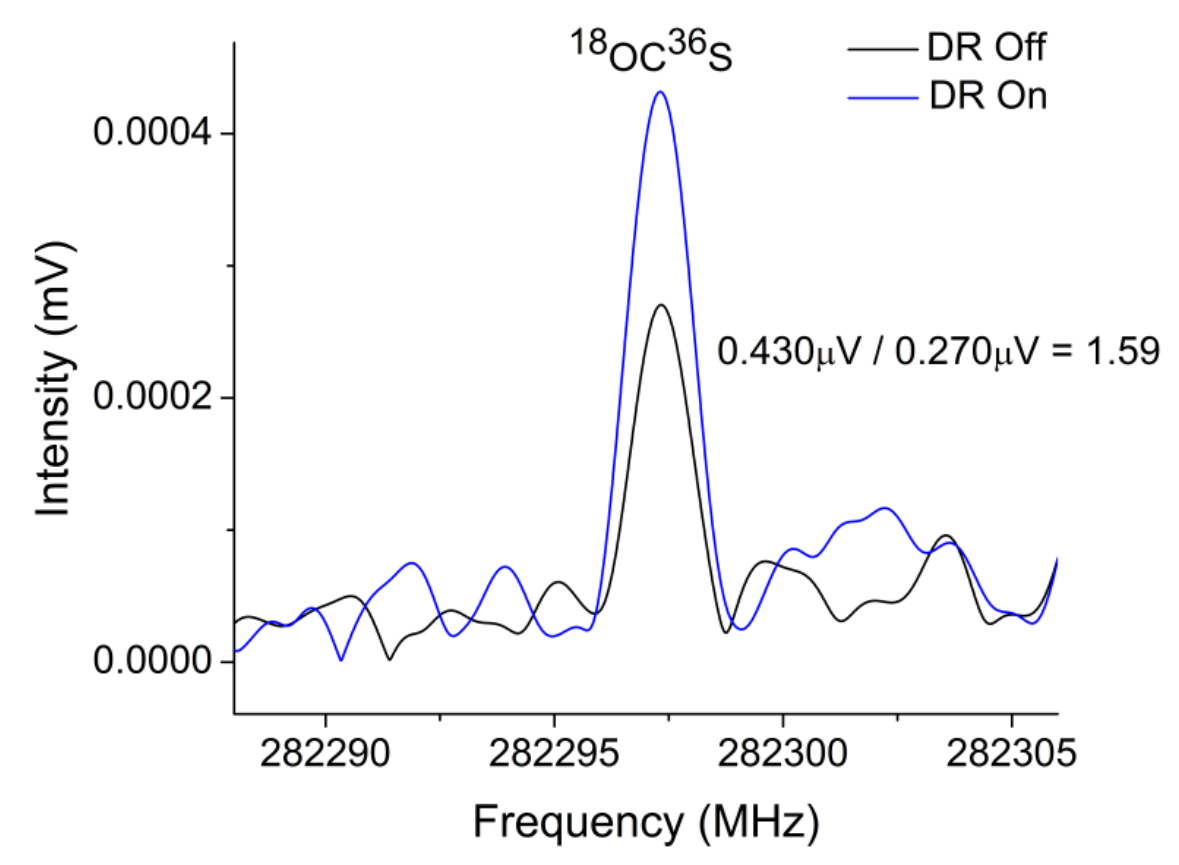

Figure 2.8: Double resonance identity verification for ${ }^{18} \mathrm{OC}^{36} \mathrm{~S}(1: 3,400,000$ ratio to main species), Probe pulse: $\mathrm{J}=26 \rightarrow \mathrm{J}=25$, Pump pulse: $\mathrm{J}=25 \rightarrow \mathrm{J}=24$. The total pressure was $10 \mathrm{~m}$ Torr and the measurement was a 10 million shot, 8 minute acquisition. This signal level corresponds to a detection level of $160 \mathrm{fmol}$. 


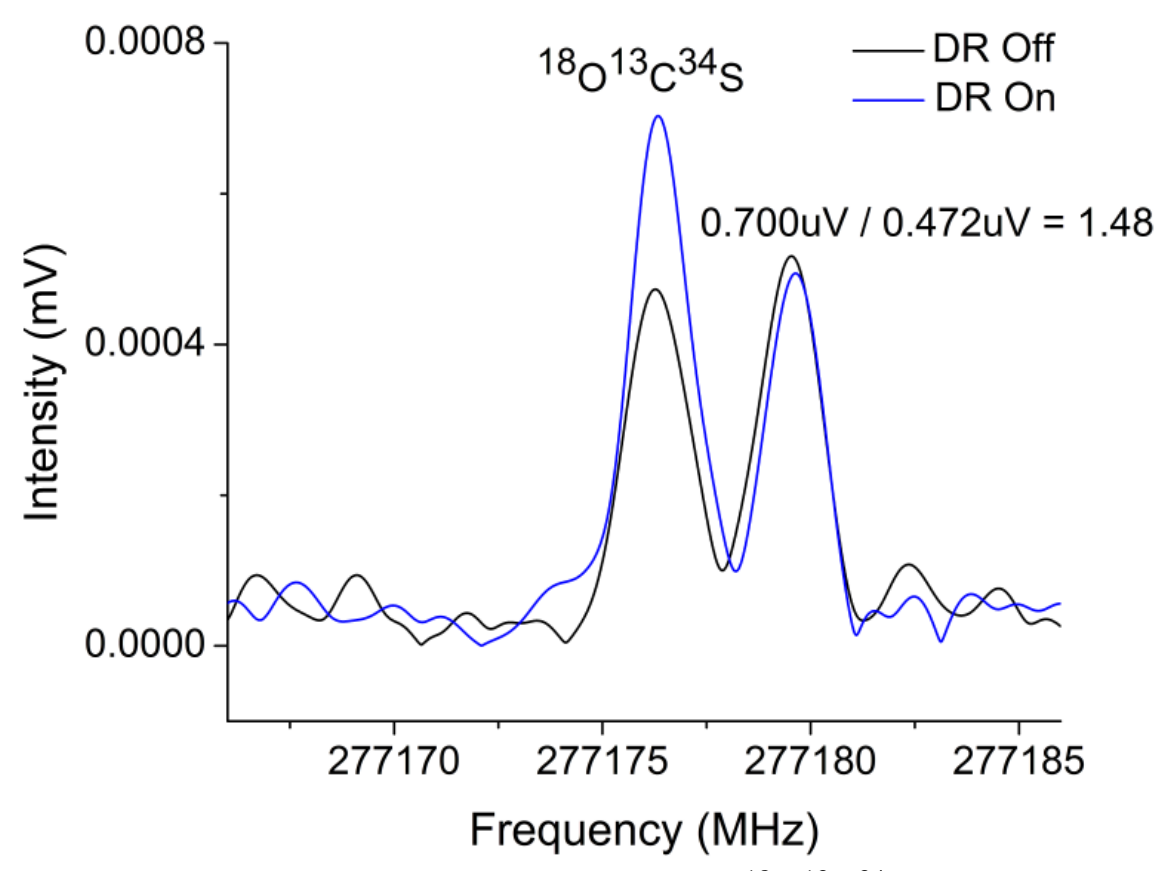

Figure 2.9: Double resonance identity verification for ${ }^{18} \mathrm{O}^{13} \mathrm{C}^{34} \mathrm{~S}(1: 1,000,000$ ratio to main species) Probe pulse: $\mathrm{J}=25 \rightarrow \mathrm{J}=24$, Pump pulse: $\mathrm{J}=26 \rightarrow \mathrm{J}=25$. The total pressure was $10 \mathrm{mTorr}$ and the measurement was a 10 million shot, 8 minute acquisition. This signal level corresponds to a detection level of $550 \mathrm{fmol}$. The nearby unassigned line is unaffected. 


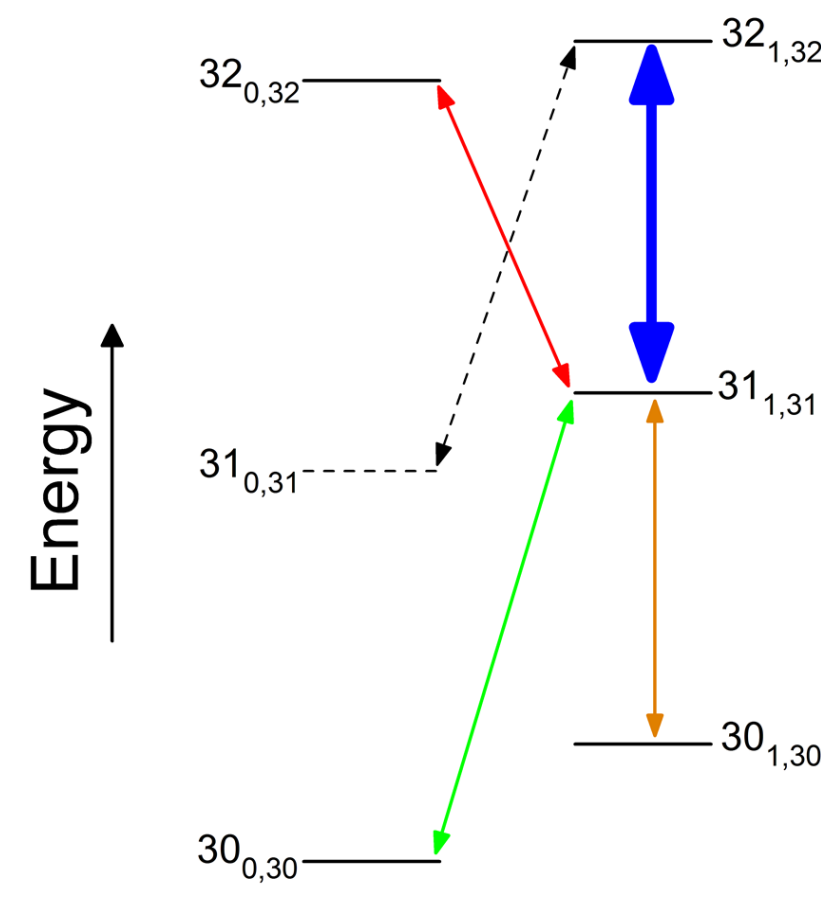

Figure 2.10: Energy level manifold for Acrolein illustrating the connected transitions to the $31(1,31)$ rotational state. 

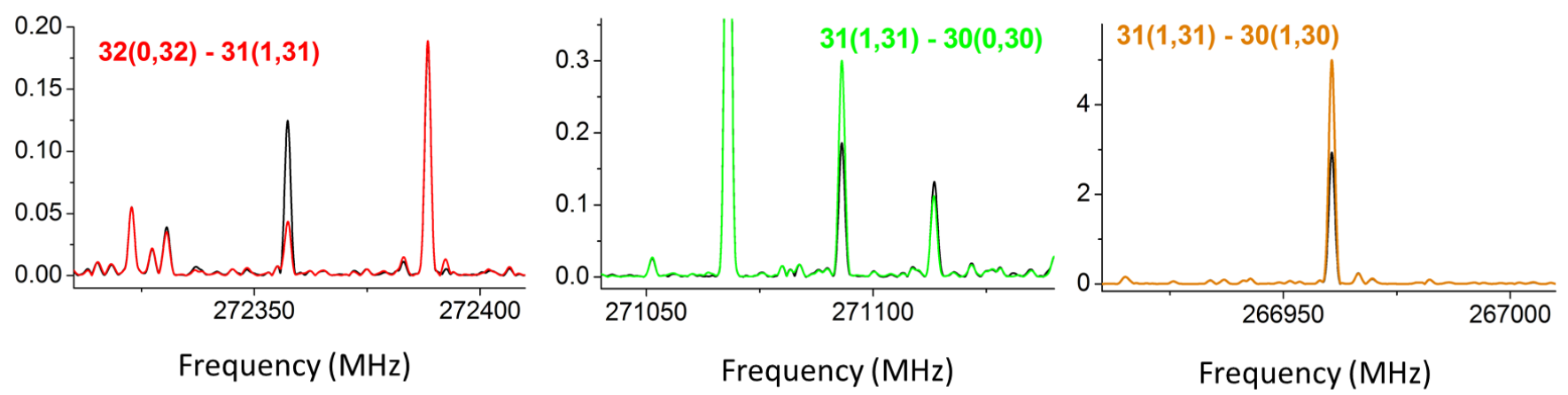

Figure 2.11: Double resonance modulation of three transitions connected to the $31(1,31)$ rotational state of acrolein. In black is the unmodulated spectrum. The three plots come from isolated $720 \mathrm{MHz}$ CP-FTmmW segments. After the pump pulse, there is a smaller population difference across the $32(0,32)-31(1,31)$ transition (red) resulting in a double resonance modulation to lower signal intensity. 
2D spectrum could be acquired in approximately 5 seconds $(50,000 \times 100 \mu \mathrm{s})$. The concept is illustrated in Fig 2.10 and Fig 2.11 using the more complex rotational energy manifold of acrolein, an asymmetric rotor. [58,59] The measurements were carried out by pairing a $720 \mathrm{MHz}$ chirped pulse (in fast mode) with a $\pi$ excitation pulse to pump the $32(1,32)-31(1,31)$ transition of Acrolein. The modulation effect is observed across the broadband spectrum for transitions connected to the $31(1,31)$ rotational energy level. Depending on how the pump pulse affects the population difference across a connected transition, the double resonance modulation will be a signal increase or signal decrease.

\section{Discussion and Conclusions}

The introduction of fast, broadband technologies for millimeter/submillimeter spectroscopy requires parallel advances for composition analysis of the high-resolution, information rich rotational spectra. The tools presented here can be used to enhance the conventional broadband spectrum analysis to include more information as an aid to the assignment process. After confidently cataloging the spectrum through broadband reference library matching, the unassigned spectrum is the next challenge, and the primary bottleneck to discovery. The first step in the analytical tools presented here would be to use a 2D double resonance scan to find connected transitions and extract an unassigned spectrum for identification. A nutation curve on a strong, non-overlapping line should follow in order indicate either a high or low dipole species and set up the pulse duration conditions for further analysis. After measuring the collisional decay rate from the Hahn echo decay profile, the narrow-band FID can be modeled to fit a Doppler decay in order to estimate the mass of the molecular carrier. The mass accuracy of $1 \mathrm{amu}$ 
can be a very important piece of information to help narrow down the assignment list. Once a guess is made it can be verified by matching a simulated spectral pattern to the extracted unassigned spectrum. A simulated spectrum can be generated from literature measurements in a different band or from a calculated molecular structure using standard computational chemistry tools in combination with spectral prediction programs. [59-61] Once the energy level manifold is well understood, double resonance identity verification can be performed to establish the molecular assignment.

The double resonance identity verification can be an important tool even in the case where the library reference spectrum has been measured. For example, if OCS exists at trace levels in a confusion limited ethyl cyanide mixture, the overlap probability is high and the assignment of OCS could be challenging since there are only three strong transitions of OCS in the spectrometer band. The double resonance modulation can prove the existence of the OCS energy level manifold and thereby confirm its presence. However, it is important to note that the double resonance measurement which results in no modulation can be just as important for applications that require low false negatives in spectrally dense mixtures.

The goal of this work is to highlight the information rich content of the millimeter-wave rotational spectrum and demonstrate how time domain spectroscopy can add information to chemical intuition in order to speed analysis. All of the measurement modes presented in this work can be fully automated and can be completed within minutes using the processing power of common desktop computers. Although the AWG based spectrometer can accommodate the new measurement modes, the analysis tools do not require an $\mathrm{AWG}$. 
Accurate spectral modeling can be challenging for millimeter/submillimeter spectroscopy without previous measurements at lower frequencies because rotational distortion effects become significant at high angular momentum states. Yet room temperature analytical chemistry applications are best addressed in the millimeter/submillimeter spectrum, and there is an interest in astrochemistry studies towards high frequency (> $1 \mathrm{THz}$ ) spectroscopy. [43] A 2D double resonance measurement would be essentially blind to the complication of spectral modeling; it is an unbiased search for connections. In combination with computational chemistry and spectral fitting programs, the time domain spectroscopy tools can help realize the potential for library free identification and provide significant relief in the identification of unknowns. As the use of instruments for millimeter-wave spectroscopy spreads into diverse chemistry applications, the cataloguing of an ever growing number of new sample matrixes will need to match pace.

\section{References for Chapter 2:}

1. R. R. Ernst, W. A. Anderson, Rev. Sci. Instru., 1966, 37, 93.

2. H. A. Gebbie, N. W. B. Stone, Infrared Physics, 1964, 4, 85.

3. M. B. Comisarow, A. G. Marshall, Chem. Phys. Lett., 1974, 25, 282.

4. J. C. McGurk, T. G. Schmalz, W. H. Flygare, J. Chem. Phys., 1974, 60, 4181.

5. J. Ekkers, W. H. Flygare, Rev. Sci. Instrum., 1976, 47 , 448.

6. T. J. Balle , W. H. Flygare, 1981, Rev. Sci. Intsr., 53, 33.

7. E. J. Campbell, L. W. Buxton, T. J. Balle, M. R. Keenan, W. H. Flygare, 1981, J. Chem. Phys., 74, 829. 
8. E. J. Campbell, L. W. Buxton, T. J. Balle, W. H. Flygare, 1981, J. Chem. Phys., 74, 813.

9. G. G. Brown, B. C. Dian, K. O. Douglass, S. M. Geyer, B. H. Pate, J. Mol. Spec., 2006, 238, 200.

10. G. G. Brown, B. C. Dian, K. O. Douglass, S. M. Geyer, S. T. Shipman, B. H. Pate, Rev. Sci. Instr., 2008, 79, 053103.

11. D. Zaleski, J. Neill, M. Muckle, N. Seifert, P. Carroll, S. Widicus-Weaver, B. Pate, J. Mol. Spec., 2012, 280, 68.

12. G. S. Grubbs, C. T. Dewberry, K. C. Etchison, K. E. Kerr, S. A. Cooke, Rev. Sci. Instr., 2007, 78, 096106.

13. S. L. Stevens, N. R. Walker J. Mol. Spec., 2010, 263, 27.

14. D. A. Obenchain, A. A. Elliott, A. L. Steber, R. A. Peebles, S. A. Peebles, C. J. Wurrey, G. A. Guirgis, J. Mol. Spec., 2010, 261, 35.

15. R. G. Bird, J. L. Neill, V. J. Alstadt, J. W. Young, B. H. Pate, D.W. Pratt, J. Phys. Chem. A, 2011, 115, 9392.

16. I. R. Medvedev, C. F. Neese, G. M. Plummer, F. C. De Lucia, Opt. Lett., 2010, 35, 1533.

17. C. Neese, I. Medvedev, G. Plummer, A. Frank, C. Ball, F. De Lucia, IEEE Sensors J., 2012, 12, 2565.

18. J. C. Pearson, B. J. Drouin, A. Maestrini, I. Mehdi, J. Ward, R H. Lin, S. Yu, J. J. Gill, B. Thomas, C. Lee, G. Chattopadhyay, E. Schlecht, F. W. Maiwald, P. F. Goldsmith, P. Siegel, 2011, Rev. Sci. Instru., 82, 093105.

19. B. J. Drouin, F. W. Maiwald, J. C. Pearson, Rev. Sci. Instr., 2005, 76, 093113.

20. R. A. Motiyenko, L. Margules, E. A. Alekseev, J. C. Guillemin, J. Demaison, J. Mol. Spec., 2010, 264, 94.

21. C. P. Endres, F. Lewen, T. F. Giesen, S. Schlemmer, D. G. Paveliev, Y. I. Koschurinov, V. M Ustinov, A. E. Zhucov, Rev. Sci. Instr., 2007, 78, 043106.

22. C. A. Gottlieb, P. C. Myers, P. Thaddeus, ApJ., 2003, 588, 655.

23. M. Winnewisser, H. Lichau, F. Wolf, J. Mol. Spec., 2000, 202, 155.

24. Z. Kisiel, E. B. Jaworska, L. Pszczolkowski, J. Mol. Spec., 1996, 177, 240. 
25. L. M. Ziurys, W. L. Barclay, M. A. Anderson, D. A. Fletcher, J. W. Lamb, Rev. Sci. Instr., 1994, 65, 1517.

26. S. Saito, M. Goto, ApJ., 1993, 410, L53.

27. T. Okabayashi, T. Mitsutoshi, J. Chem. Phys., 1993 99, 3268.

28. A. L. Steber, B. J. Harris, J. L. Neill, B. H. Pate, J. Mol. Spec., 2012, 280, 3.

29. J. L. Niell, B. J. Harris, A. L. Steber, K. O. Douglas, D. F. Plusquellic, B. H. Pate, Opt. Expres., 2013, 21, 19743.

30. E. Gerecht, K. Douglass, D. Plusquellic, Opt. Lett., 2011, 19, 8973.

31. G. B. Park, A. H. Steeves, K. Kuyanov-Prozument, J. L. Neill, R. W. Field, J. Chem. Phys., 2011, 135, 024202.

32. B. J. Harris, A. L. Steber, B. H. Pate, Rev. Sci. Instr., in preparation.

33. S. Albert, D. T. Petkie, R. P. A. Bettens, S. P. Belov, F. C. De Lucia, Anal. Chem. 1998, 70, 719A.

34. F. C. De Lucia, J. Opt. Soc. Am. B, 2004, 21, 1273.

35. I. R. Medvedev, M. Behnke, F. C. De Lucia, Analyst, 131, 1299.

36. D. N. Friedel, L. E. Snyder, B. E. Turner, A. Remijan, ApJ, 2004, 600, 234.

37. A. O. H. Olofsson, C. M. Persson, N. Koning, P. Bergman, P. F. Bernath, J. H. Black, U. Frisk, W. Geppert, T. I. Hasegawa, A. Hjalmarson, A\&A, 2007, 476, 791.

38. N. Kaifu, M. Ohishi, K. Kawaguchi, S. Saito, S. Yamamoto, T. Miyaji, K. Miyasawa, S. Ishikawa, C. Noumaru, S. Harasawa, PASJ, 2004, 56, 69.

39. N. A. Patel, K. Y. Young, C. A. Gottlieb, P. Thaddeus, R. W. Wilson, K. M. Menten, M. J. Reid, M. C. McCarthy, J. Cernicharo, J. He, S. Brunken, D. Trung, E. Keto, ApJS, 2011, 193, 17.

40. E. D. Tenenbaum, J. L. Dodd, , S. N. Milam, N. J. Woolf, L. M. Ziurys, ApJ, 2010, 190, 348 .

41. J. M. Hollis, A. J. Remijan, P.R., Jewell, R. J. Lovas, The GBT PRIMOS project: Searching for Our Molecular Origins. 
42. N. R. Crocket, E. A. Bergin, S. Wang, D. C. Lis, T. A. Bell, G. A. Blake, A. Boogert, B. Bumble, S. Cabrit, E. Caux, $A \& A, 2010,521$, L21.

43. J. Neill et al. 2014, submitted.

44. H. S. P. Muller, F. Shloder, J. Stutzki, G. Winnewisser. J. Mol. Struct., 2005, 742, 215.

45. H. M. Pickett, R. L. Poynter, E. A. Cohen, M. L. Delitsky, J. C. Pearson, H. S. P. Muller, J. Quant. Spectrosc. \& Rad. Transfer, 1998, 60, 883.

46. F. J. Lovas, R. A. Dragoset, J. Phys. Chem. Ref. Data, 2004, 33, 177.

47. S. Green, J. Chem. Phys., 1978, 69, 4076.

48. S. M. Fortman, I. Medvedev, C. Neese, F. C. De Lucia, ApJ, 2010, 725, 1682.

48. H. Harde, R. A. Cheville, D. Grischkowsky, J. Phys. Chem. A, 1997, 101, 3646.

49. E .L. Hahn, Phys. Rev., 1950, 80, 580.

50. R. G. Brewer, R. L. Shoemaker, Phys. Rev. Lett., 1964, 27, 631.

51. W. E. Hoke, D. R. Bauer, J. Ekkers, and W. H. Flygare, J. Chem. Phys., 1976, 64, 5276.

52. H. Mader, J. Ekkers, W. Hoke, W. H. Flygare, J. Chem. Phys., 1975, 62, 4380.

53. A. P. Columbo, Y. Zhou, K. Prozument, S. L. Coy, R. W. Fields, J. Chem. Phys., 2013, 138, 014301.

54. S. Matton, F. Rohart, R. Bocquet, G. Mouret, D. Bigourd, A. Cuisset, and F. Hindle, J. Mol. Spec., 2006, 239, 182.

55. L. A. Surin, D. N. Fourzikov, F. Lewen, B. S. Dumesh, G. Winnewisser, A. R. W. McKellar, J. Mol. Spec., 2003, 222, 93.

56. K. Kubo, T. Furuya, S. Saito, J. Mol. Spec., 2003, 222, 256.

57. supplementary information.

58. A. I. Jaman, R. Bhattacharya, J. Atomic. Mol. Opt. Phys.I, 2012, 2012, 363247.

59. H .M. Pickett, J. Mol. Spectrosc., 1991 148, 371.

60. M. J. Frish et al., Gaussian 09, Gaussian Inc., Wallingford CT, 2009. 
61. V. V. Ilyushin, Z. Kisieal, L. Pszczolowski, H. Mader, J. T. Hougen, J. Mol. Spec., 2010, 256, 26. 


\begin{tabular}{|c|c|c|c|}
\hline \multicolumn{4}{|c|}{$\begin{array}{l}\text { Supplementary Table 2.1: CPFTmmW line centers } \\
\text { for OCS isotopologues }\end{array}$} \\
\hline Species & Catalogue $^{\mathrm{a}}$ & Measured ${ }^{b}$ & OMC \\
\hline \multicolumn{4}{|l|}{$\mathrm{OC}^{34} \mathrm{~S}$} \\
\hline $\bar{J}=22-21$ & 260991.81 & 260991.81 & 0.00 \\
\hline $\mathrm{J}=23-22$ & 272849.96 & 272849.93 & -0.03 \\
\hline $\mathrm{J}=24-23$ & 284707.29 & 284707.36 & 0.07 \\
\hline \multicolumn{4}{|l|}{$\mathrm{O}^{13} \mathrm{CS}$} \\
\hline $\mathrm{J}=22-21$ & 266669.38 & 266669.37 & -0.01 \\
\hline $\mathrm{J}=23-22$ & 278785.34 & 278785.33 & -0.01 \\
\hline $\mathrm{J}=24-23$ & 290900.59 & 290900.57 & -0.02 \\
\hline \multicolumn{4}{|l|}{$\mathrm{OC}^{33} \mathrm{~S}$} \\
\hline $\bar{J}=22-21$ & 264162.19 & 264162.19 & 0.00 \\
\hline $\mathrm{J}=23-22$ & 276164.30 & 276164.30 & 0.00 \\
\hline $\mathrm{J}=24-23$ & 288165.75 & 288165.72 & -0.03 \\
\hline \multicolumn{4}{|l|}{${ }^{18} \mathrm{OCS}$} \\
\hline$\overline{\mathrm{J}=23-22}$ & 262368.30 & 262368.31 & 0.01 \\
\hline $\mathrm{J}=24-23$ & 273770.55 & 273770.49 & -0.06 \\
\hline $\mathrm{J}=25-24$ & 285172.05 & 285172.05 & 0.00 \\
\hline \multicolumn{4}{|l|}{$\mathrm{O}^{13} \mathrm{C}^{34} \mathrm{~S}$} \\
\hline $\mathrm{J}=22-21$ & 260063.53 & 260063.55 & -0.02 \\
\hline $\mathrm{J}=23-22$ & 271879.48 & 271879.48 & 0.00 \\
\hline $\mathrm{J}=24-23$ & 283694.74 & 283694.74 & 0.00 \\
\hline \multicolumn{4}{|l|}{${ }^{17} \mathrm{OCS}$} \\
\hline $\mathrm{J}=23-22$ & 270589.99 & 270590.00 & 0.01 \\
\hline $\mathrm{J}=24-23$ & 282349.31 & 282349.31 & 0.00 \\
\hline $\mathrm{J}=25-24$ & 294107.93 & 294107.94 & 0.01 \\
\hline \multicolumn{4}{|l|}{$\mathrm{OC}^{36} \mathrm{~S}$} \\
\hline $\bar{J}=23-22$ & 266727.97 & 266728.01 & 0.04 \\
\hline $\mathrm{J}=24-23$ & 278319.48 & 278319.52 & 0.04 \\
\hline $\mathrm{J}=25-24$ & 289910.30 & 289910.33 & 0.03 \\
\hline \multicolumn{4}{|l|}{${ }^{18} \mathrm{OC}^{34} \mathrm{~S}$} \\
\hline $\mathrm{J}=24-23$ & 266818.85 & 266818.90 & 0.05 \\
\hline $\mathrm{J}=25-24$ & 277931.02 & 277931.05 & 0.03 \\
\hline $\mathrm{J}=26-25$ & 289042.54 & 289042.59 & 0.05 \\
\hline \multicolumn{4}{|l|}{$\underline{\mathrm{O}^{13} \mathrm{C}^{33} \mathrm{~S}}$} \\
\hline $\mathrm{J}=22-21$ & 263266.85 & 263266.93 & 0.08 \\
\hline $\mathrm{J}=23-22$ & 275228.29 & 275228.35 & 0.06 \\
\hline $\mathrm{J}=24-23$ & 287189.03 & 287189.09 & 0.06 \\
\hline \multicolumn{4}{|l|}{${ }^{18} \mathrm{O}^{13} \mathrm{CS}$} \\
\hline $\bar{J}=23-22$ & 261734.01 & 261734.00 & 0.01 \\
\hline $\mathrm{J}=24-23$ & 273108.65 & 273108.62 & -0.03 \\
\hline $\mathrm{J}=25-24$ & 284482.57 & 284482.62 & 0.05 \\
\hline $\mathrm{OMC} \mathrm{rr}$ & & & 0.04 \\
\hline
\end{tabular}

a) from the CDMS catalogue, see reference 44

b) measurements in triplicate 


\title{
Chapter 3. Mixture Analysis - Solutions
}

\author{
Direct Head Space Analysis by Chirped-Pulse Fourier Transform \\ Millimeter-wave Spectroscopy
}

\section{Introduction:}

Despite decades of application to molecular structure research and composition studies of the interstellar medium, molecular rotational spectroscopy has not experienced the broad industrial application that essentially every other type of molecular spectroscopy has. Instruments for NMR, EPR, IR, UV/vis, and X-ray spectroscopy all have their own analytical product lines and probe molecular dynamics using the entire electromagnetic spectrum from radio frequency to x-rays. However, there is a frequency gap unaddressed by current analytical instruments that are used to probe pure molecular rotation quantum transitions in the microwave to $\mathrm{THz}$ region. Analytical chemistry literature has drawn attention to this frequency gap. [1,2] Historically, molecular rotational spectroscopy has been used as a powerful tool in physical chemistry and astronomy. Spectroscopists have used it to study molecular structure of weakly bound complexes and transient molecules. It has long been promised as a superior technique for absolute chemical recognition in analytical chemistry due to the direct relationship between the spectral pattern and the three dimensional mass distribution of the molecule through the principal moments of inertia. [3-5] The selectivity of rotational spectroscopy relies on the unambiguous rotational fingerprint and the high-resolution. Together, these two features enable the direct analysis of complex mixtures with extremely low false positives and has been its strength as an analytical science for astrochemistry. 
There has been an effort over the decades to build commercially viable instruments based on rotational spectroscopy. The first effort was by Hewlett-Packard in the 1970s with the Stark modulated microwave spectrometer. [6,7] The cost, performance, and form factor did not reach the balance to make it competitive with other techniques so it was only marginally adopted in academic research labs. [8] In the 1980s, the first Fourier transform cavity based instruments were introduced and marked a milestone for microwave spectroscopy development, which has since been totally supplanted by Fourier transform methods, over direct absorption. [9-11] It became the next platform for miniaturization and application to gas sensing. [12,13] Concept measurements showed promising sensitivity but the molecular beam technique required expensive carrier gases and slow, stepped tuning. For broadband screening of a sample matrix, the mini-FT-MW spectrometer was not practical.

Rotational spectroscopy encompasses a very wide frequency space. One major disadvantage that previous attempts at commercialization suffered from was the fact that the microwave spectrum $(<40 \mathrm{GHz})$ is not ideally suited for detecting the common molecules of interest in room temperature, analytical chemistry settings. At room temperature, the rotational spectrum of common volatiles peaks in intensity at millimeter/submilllimeter wavelength. Such was the principle behind the development of FASSST spectroscopy introduced in the late 1990s. [14] Numerous discussions about the potential of these spectrometers and their strengths for new gas sensing capabilities have been generated out of the group that developed FASSST spectroscopy. [15-18] Advances in computing and solid-state millimeter/submillimeter light sources (technology to address the $\mathrm{THz}$ "gap") have enabled the design of modern direct 
absorption spectrometers that have been converging towards commercially viable designs. However, with the introduction of broadband chirped pulse Fourier transform microwave (CP-FTMW) spectrometers, molecular rotational spectroscopy has gone through another transformation in speed and sensitivity that is typical of other techniques that supplant direct absorption with Fourier transform methods. [e.g. 19]

Since its introduction in 2006, CP-FTMW has become a favored technique for new microwave spectrometers due to its unmatched capabilities for broadband acquisition speed. [20-26] It has been extended to millimeter-wave spectroscopy in recent years and has resulted in the design of a new CP-FTmmW spectrometer which is used in this work. [27-31] CP-FTmmW brings a 10,000 fold speed enhancement over its modern direct absorption counterparts and is the driver for sensitivity that is relevant for industrial scale analytical chemistry. A 10 minute measurement can produce a dynamic range of $10^{6}$. By other rotational spectroscopy techniques this would take at least 20,000 minutes (or 14 days). [31, 32]

The fundamental benefits of using molecular spectroscopy include molecule specific detector response, direct analysis of mixtures, and non-destructive measurement. In the last decade, spectroscopic instruments have gone through major transformations and delineations driven by the Process Analytical Technology (PAT) and Quality by Design (QBD) initiatives of the FDA. [33,34] A major theme in analytics for chemical manufacturing has been to monitor critical processes as they are happening so that quality is designed into the final product. The effort has created the need for automated, fast, non-destructive instruments. 
With the introduction of CP-FTmmW and the wide-spread interest for spectroscopy technology adoption, now is the time for renewing the value proposition of pure rotational spectroscopy. In order to ensure the long-lived free rotation of molecules, pure rotational spectroscopy takes place at low pressures in gas mixtures. Compressed gas mixtures are the usual matrix of choice for benchmark measurements; $[2,12,13,16]$ however, the low pressure requirements of rotational spectroscopy enable the analysis of solution headspace and sublimated solids. Residual solvents in solution or off-gassed in solids is an important measurement that has not been sufficiently highlighted in the literature regarding rotational spectroscopy for analytical chemistry. In this work, we benchmark the performance of the CP-FTmmW spectrometer with the use case of headspace analysis, which has a more direct implication to use of rotational spectroscopy for solvent analysis of chemical products or reaction mixtures. Methods for VOC analysis covering air, soil, and water include detection of a standard list of approximately 100 volatiles. [35-41] Fast eluting, high vapor pressure gases pose a challenge for time resolution in column chromatography. A standard mixture of 6 high vapor pressure gases is commercially available and will serve as the sample for this study. A regulated method for VOC analysis in water is the EPA method 524 which includes purge and trap, gas chromatography-mass spectrometry (GC-MS) for detection. [35] Detection limits and measurement times for direct CP-FTmmW headspace analysis are compared to EPA method 524 in the discussion section. The goal of this work is to highlight CP-FTmmW advantages that enable an information rich volatile mixture analysis by simple, fast methods. 
Commercial analytics for gas/solvent analysis:

GC-MS has become a very powerful tool for chemical detection, but it is generally incompatible with the PAT initiative. Detectors, which rely on chemical separation, have the advantage of having broad composition analysis capability and low detection limits; however it comes with significant sample pre-conditioning and is calibration intensive. Columns can be expensive consumables and the separation step itself is not universal. As described above, time resolution of fast eluting gases on a gas chromatographic column can be difficult, and that is only one challenge. For GC-MS, solvent-solute interaction can cause interference problems and corrupt the fractionation spectrum where solutes have a similar mass to the solvent. As a result, in environmental studies, volatiles analyses are typically run above 32 amu (methanol a common solvent and mass $32 \mathrm{amu}$ ). Even where resolution and solvent interference are not a problem, any filtering method involves interaction of the analyte mixture with a material before detection. For corrosive or unstable species, interaction with a material such as a sorbent or column can cause unwanted derivatization. For some applications, the challenge is maintaining sample integrity during the pre-conditioning. As a result, derivatization is sometimes used by design to preclude the filtering and detection steps requiring more complex chemistry and more operator attention. [42] Ideally, direct detection of the analyte is desired and molecular spectroscopy is the way to do this.

The simplicity of infrared spectroscopy has made it a popular technique for process monitoring, and instruments that probe vibrational energies have proliferated in recent years. Chemometric techniques have been developed for deconvolution of a mixture's infrared spectrum into its component parts for estimation of the composition 
without chemical filtering and have become an independent field of study. [43] The modeling process takes years and the results enable handheld identity verification. [44] However for sensitive mixture analysis, the resolution in the IR spectrum limits the dynamic range. Cavity based instruments for gas detection in the mid IR can be very sensitive, but the chemical coverage of a single instrument is limited. [45,46] Laser sources for mid IR are typically tunable across a few wavenumbers. [47] In some cases, multiple lasers can be used to address other narrow bandwidths, but the chemical coverage is still highly limited. The PAT initiative has even driven the miniaturization of FT-NMR and the development of sampling modules for online monitoring of small scale reactions. [48]

Molecular rotational spectroscopy can be used for broad composition analysis of volatile molecules with dipole moments and achieve molecular selectivity that is unmatched by any other technique in analytical chemistry. In general, spectroscopy techniques have drawbacks for sensitivity or broad chemical coverage. This tradeoff is not the case for molecular rotational spectroscopy using the CP-FTmmW spectrometer. CP-FTmmW combines the fundamental selectivity of rotational spectroscopy with broadband analytical speed for high dynamic range mixture analysis.

\section{Experimental:}

\section{A. Spectrometer}

The spectrometer is described in detail in a previous publication. [31] To summarize here, the spectrometer derives its chirped pulses from a dual channel, $12 \mathrm{GS} / \mathrm{s}$ arbitrary waveform generator (AWG). Two spectrometer modes are used in this work. 
In broadband, high dynamic range (HDR) mode, channel one generates a series of chirped pulses at low bandwidth, each with measurement cycles of $2 \mu$ s $(0.1 \mu$ s excitation pulses followed by a $1.9 \mu$ s "off" time), which together address the bandwidth of the spectrometer. The second channel of the AWG generates, single frequency, local oscillators (LO) which step in sequence with the series of chirped pulses every $2 \mu$ s so that the intermediate frequency (IF) of the heterodyne receiver is maintained between $720-1440 \mathrm{MHz}$. Both channels of AWG output from 2-3.5 GHz are upconverted off of a common $8.8 \mathrm{GHz}$ PDRO for a final input of $10.8-12.3 \mathrm{GHz}$ to the active multiplier chains (AMCs). The AMC source is Virginia Diodes Inc., x24, high power $(20-45 \mathrm{~mW})$ multiplier chain with output from $260-295 \mathrm{GHz}$. The receiver is comprised of a subharmonic mixer driven bay an LO from a x12 AMC, low noise amplification, and bandpass filters.

The time domain free induction decay (FID) of the molecular emission following an excitation pulse is digitized on a $4 \mathrm{GS} / \mathrm{s}$ high-speed digitizer equipped with an FPGA accumulator for real-time signal averaging of data traces up to $131 \mu \mathrm{s}$. For this work, high dynamic range (HDR) mode was used for broadband spectral acquisition which consists of $1500,24 \mathrm{MHz}$ chirps. Sixty two measurement cycles, or $1440 \mathrm{MHz}$ of bandwidth, can be accumulated in real-time. The full band spectrum is recovered by concatenating the magnitude fast Fourier transform of each individual FID.

For dedicating the measurement time to specific analytes of interest, targeted mode is used. The targeted mode waveform consists of a single frequency, transform limited excitation pulse with a $2 \mu$ s measurement cycle $(0.2 \mu$ s excitation pulse followed 
by a $1.8 \mu \mathrm{s}$ "off" time) and a paired local oscillator. Targeted spectral lines are monitored consecutively with all FIDs accumulated in the digitizer in real-time.

The sample cell is a free-space steel tube $65 \mathrm{~cm}$ in length and $3.8 \mathrm{~cm}$ in diameter with an approximate volume of 1L and enclosed by polytetrafluoroethylene (PTFE) focal lenses which collimate a Gaussian beam to approximately $2 \mathrm{~cm}$ in diameter. Pressure is monitored with a Baratron capacitance manometer and the vacuum pump is a compact turbo, mechanical pump combo. All components are powered from standard $120 \mathrm{~V}$ wall outlets and the spectrometer is totally cryogen-free.

\section{B. Distinctions for CP-FTmmW in Rotational Spectroscopy}

The recent enabling technologies for CP-FTmmW are essentially the same as those for fast millimeter absorption spectroscopy. 1) The availability of high frequency, broadband, solid-state millimeter sources, 2) Advances in high-speed digital electronics and computation. However, there are key distinctions to why CP-FTmmW represents a significant advance and perhaps the last step for developing a new widespread adoption path for rotational spectroscopy.

CP-FTmmW uses high power AMCs (approximately $50 \mathrm{~mW}$ vs $0.5 \mathrm{~mW}$ ) to improve measurement efficiency. Discussions on designs for absorption spectrometers usually include the need to attenuate and monitor the source power so that the absorption associated with a molecular rotational transition does not saturate. [15] To a chirped pulse spectrometer, attenuating the power is the equivalent of throwing away sensitivity. By applying the source power across a broad frequency sweep all of the power can be utilized for measurement because the source brightness is reduced per frequency element 
without overall attenuation. As a result, CP-FTmmW can benefit from the new high power AMCs. With nearly 10,000 times the power, CP-FTmmW spectrometers translate the power to nearly 10,000 times the speed.

It was also recognized in early literature that the number of data points in a broadband rotational spectrometer posed challenges for fast scanning and fast processing. For an absorption spectrometer, improving the point resolution means taking more measurements; however, in a chirped measurement the entire frequency axis is constructed in the Fourier transform. Point resolution is decided by computation and improved by "zero padding", a common signal processing technique used in FT-NMR. [49] In the chirped pulse, "segmented" spectrum up to $720 \mathrm{MHz}$ of the spectrum is acquired at once. The first CP-FTmmW spectrometer utilized a $35 \mathrm{GHz}$ frequency sweep (the full spectrometer band, $260-295 \mathrm{GHz}$ ) in one acquisition cycle using a high cost, high performance $100 \mathrm{GS} / \mathrm{s}$ digitizer. [27] The segmented technique is used in the current CP-FTmmW spectrometer in order to maximize duty cycle and reduce cost. In principle, segmenting the spectrum influences no change in the broadband sensitivity. [31]

Similar to FT-NMR, CP-FTmmW is an emission spectroscopy. Rather than detecting the drop in transmitted light power versus frequency and construct the spectrum point by point, the coherent, multicolor, free induction decay of the molecular signal is detected against zero light background. In contrast to direct absorption methods, CP-FTmmW requires no baseline processing.

In a recent description of a rack mountable millimeter absorption spectrometer, the frequency agility of modern electronics and millimeter sources was utilized to 
demonstrate a "snippit" approach to gas sensing, whereby the measurement devotes all of its time to a set of narrow frequency bands in order to monitor a specific subset of molecules. [16] The same concept frames the "targeted" mode presented here. However, since the CP-FTmmW spectrometer uses an AWG, targeted transitions do not have to be monitored to low limits, one at a time. The AWG transitions every $2 \mu$ s to a new excitation pulse so that the molecular FIDs can be round robin averaged in the digitizer. This way, each line will represent the same average conditions throughout the measurement. There is also a signal enhancement that comes from the ability to coherently drive molecular transitions to the optimal $\pi / 2$ maximum population inversion and is described in the results section.

\section{Sampling}

The sample for analysis is the VOC mix \#6 Supelco standard, packaged as $1.5 \mathrm{~mL}$ in an amber ampule (48799-U). The standard consists of 6 solutes dissolved in methanol at $2000 \mu \mathrm{g} / \mathrm{mL}$ (see Table 3.1). Before the mixture analysis, the pure library reference spectra of four readily available components in the mixture were recorded in HDR mode: choromethane at 5 mTorr (Aldrich 295507), chloroethane at $5 \mathrm{mTorr}$ (Aldrich 295310), trichlorofuoromethane at $5 \mathrm{mTorr}$ (Aldrich $254991>99.5 \%$ ), and methanol at $1 \mathrm{mTorr}$ (Macron 3041-06) . Chloroethane and chloromethane were in compressed Aldrich Sure/Pac ${ }^{\mathrm{TM}}$ containers. The pure liquid headspace served as the reference sample for trichlorofluoromethane (a high vapor pressure liquid at room temperature) and methanol. Additionally, each component in the VOC mix is available as an individual standard dissolved in methanol at various concentrations (Chloromethane $200 \mu \mathrm{g} / \mathrm{mL}$ 
Table 3.1: EPA VOC mix \#6 Components and Properties

\begin{tabular}{llcccc}
\hline Chemical & Formula & $\begin{array}{c}\text { Mass } \\
(\mathrm{amu})\end{array}$ & $\begin{array}{c}\text { Henry's Law } \\
\text { Constant } \\
(\mathrm{atm} \mathrm{m} / \mathrm{mol})\end{array}$ & $\begin{array}{c}\text { Net Dipole } \\
\text { Moment }\end{array}$ & $\begin{array}{c}\mathrm{S} / \mathrm{N} \\
100 \mathrm{mg} / \mathrm{L}\end{array}$ \\
\hline Chloromethane & $\mathrm{ClCH}_{3}$ & 50.49 & $8.82 \mathrm{E}-03^{\mathrm{b}}$ & $1.9^{\mathrm{e}}$ & $26,000: 1$ \\
Chloroethane & $\mathrm{ClCH}_{3} \mathrm{CH}_{3}$ & 64.51 & $1.11 \mathrm{E}-02^{\mathrm{b}}$ & $2.1^{\mathrm{e}}$ & $6000: 1$ \\
Bromomethane & $\mathrm{BrCH}_{3}$ & 94.94 & $6.24 \mathrm{E}-03^{\mathrm{c}}$ & $1.8^{\mathrm{f}}$ & $6000: 1$ \\
Vinyl Chloride & $\mathrm{ClCHCH}_{3}$ & 62.50 & $2.70 \mathrm{E}-02^{\mathrm{c}}$ & $1.5^{\mathrm{e}}$ & $5000: 1$ \\
Dichlorodifluoromethane & $\mathrm{Cl}_{2} \mathrm{~F}_{2} \mathrm{C}$ & 120.91 & $2.25 \mathrm{E}-01^{\mathrm{c}}$ & $0.5^{\mathrm{e}}$ & $25: 1$ \\
Trichlorofluoromethane & $\mathrm{Cl}_{3} \mathrm{FC}$ & 137.37 & $9.70 \mathrm{E}-02^{\mathrm{d}}$ & $0.5^{\mathrm{e}}$ & $35: 1$ \\
Methanol $^{\mathrm{a}}$ & $\mathrm{CH}_{3} \mathrm{OH}$ & 32.04 & $4.55 \mathrm{E}-06^{\mathrm{c}}$ & $1.7^{\mathrm{e}}$ & $7000: 1$ \\
\hline
\end{tabular}
a) Diluent
b) Reference 50 (Gosset et al. 1987)
c) Reference 51 (USEPA 1982)
d) Reference 52 (Nelson et al. 1967)
e) Reference 53 (Gray et al. 1984) 
Supelco 48622, Chloroethane $200 \mu \mathrm{g} / \mathrm{mL}$ Supeclo 4-8626, Bromomethane $200 \mu \mathrm{g} / \mathrm{mL}$ Supelco 4-8624, Vinyl Chloride $2000 \mu \mathrm{g} / \mathrm{mL}$ Supeclo 500976, Trichlorofluoromethane $5000 \mu \mathrm{g} / \mathrm{mL}$ Supelco 4-0047, Dichlorodifluoromethane $5000 \mu \mathrm{g} / \mathrm{mL}$ Supelco 4-0346). For identification in the mixture, the headspace spectrum of each individual standard was measured after dilution of $1 \mathrm{~mL}$ of the mixture to $10 \mathrm{~mL}$ in water (Macron 6795-10). The stock mixture solution was prepared at $100 \mu \mathrm{g} / \mathrm{mL}$ by diluting $1 \mathrm{~mL}$ of the standard mixture to $20 \mathrm{~mL}$ with water. Detection limits were determined by consecutive measurements of serial dilutions by 10 of the stock mixture with water.

The vapor/liquid equilibration vessel is a glass-metal Kovar sealed tube which mates to standard $1 / 4$ inch Swagelok on one end and is Viton ${ }^{\circledR}$ o-ring sealed to a glass, sample container (approximately $5 \mathrm{~mL}$ of volume) on the other end. The liquid is sealed in the equilibration vessel, frozen with liquid nitrogen and the atmosphere is evacuated. The sample is then allowed to reach room temperature and equilibrate for 10 minutes. After which, a 10 mTorr aliquot is introduced to the spectrometer sample cell. Both an HDR, broadband measurement is acquired and a targeted measurement, which probes two lines of each of the six species (including methanol) for a total of 14 excitation pulses (28 $\mu$ s data trace).

\section{Results}

\section{A. Broadband Mixture Survey}

The full-band spectrum from $260-295 \mathrm{GHz}$ of the EPA VOC mix \#6 headspace at $100 \mu \mathrm{g} / \mathrm{ml}$ is shown in Fig 3.1. The measurement took approximately 10 minutes and is comprised of two 100,000 average spectra averaged in the frequency domain, one set 
with frequency sweeps running up in frequency and set running down. Chloromethane, chloroethane, vinylchloride, bromomethane, and methanol all appear at high signal-to-noise suitable for trace detection (see Table 3.1). Features for these molecules are indicated in the full band spectrum of Fig 3.1. Trichlorofluoromethane and dichlorodifluoromethane are significantly heavier than the other volatiles in the mix. The large rotational partition functions and lower dipole moments of the two heavier volatiles contribute to a significantly weaker and denser room temperature, $260-295 \mathrm{GHz}$ spectrum. Practical instrument limitations aside, it is more favorable to analyze heavier molecules at lower frequency bands where the Boltzmann peak spectral intensity lies. Trichlorofluoromethane and dichlorofluoromethane are visible in the expanded plots of Fig. 3.1 at greater than 10:1.

The full band spectrum at the top of Fig. 3.1 conveys the chemical coverage addressed in the $35 \mathrm{GHz}$ bandwidth, however it hardly justifies the high-resolution information content. The subplots in Fig. 3.1 convey the relative silence of the large bandwidth spectrum even with the mixture content. Spectral patterns and individual lines are well resolved. Considering a broadband spectrum of $35 \mathrm{GHz}$ and a line center accuracy of $100 \mathrm{kHz}$, there are on the order of $10^{5}$ independent data channels. Comparison of the intensity axes of the subplots, highlights the dynamic range addressed in the 10 minute measurement. The intensity scale of the chloromethane plot is 1,000 times larger than that of the dichlorodifluoromethane plot where the baseline noise floor becomes visible (approximately $400 \mathrm{nV}$ ). Trichlorofluoromethane and 

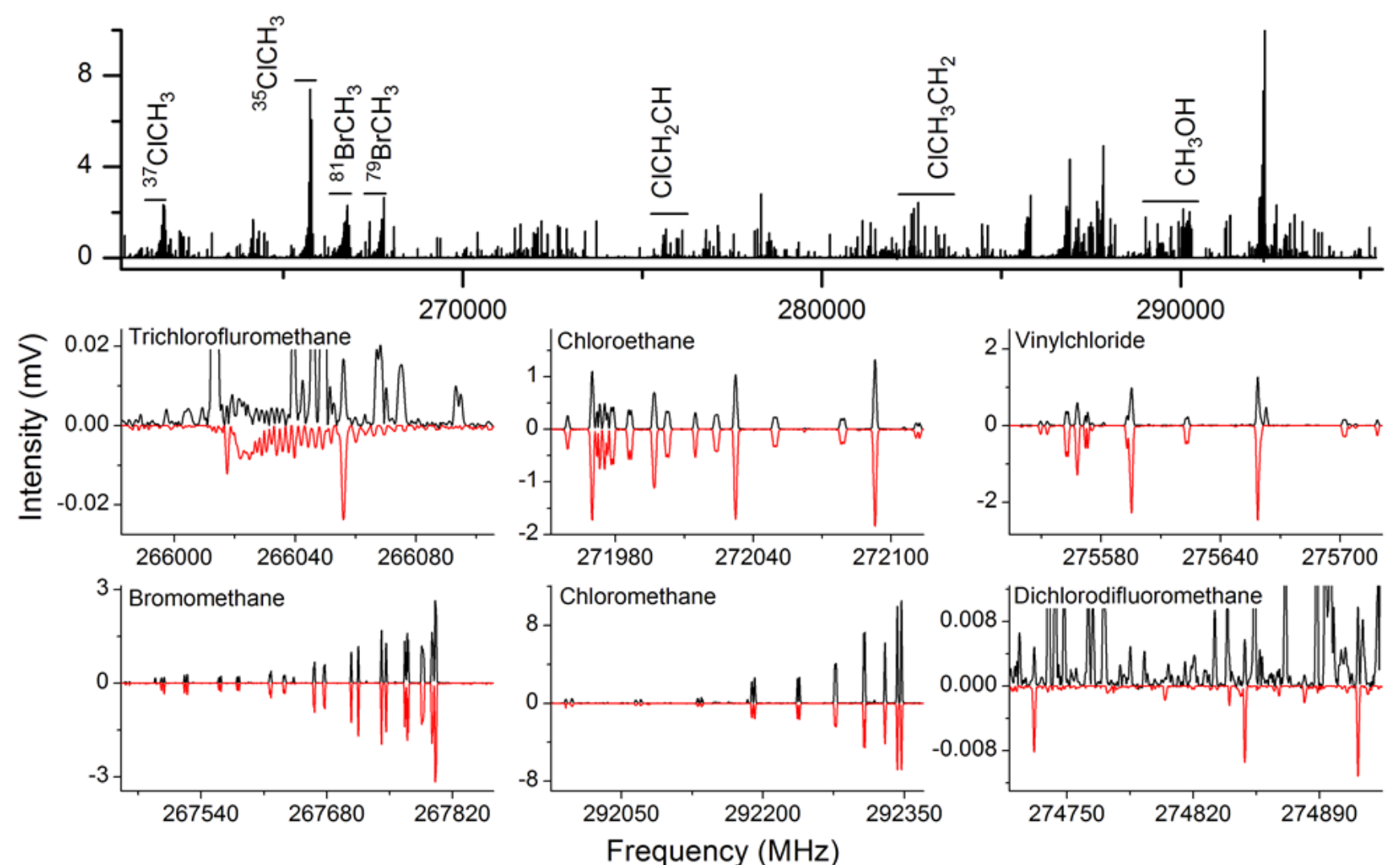

Figure 3.1: The full-band spectrum of 10 mTorr of headspace over $100 \mu \mathrm{g} / \mathrm{mL}$ solution of EPA VOC mix \#6 (top). The diluent is 20:1 water/methanol. The plots below isolate spectral regions and illustrate the selectivity of the gas mixture millimeter spectrum. Reference spectra of single component solutions are shown in red. 
dichlorodifluoromethane signals are clearly identified in the presence of signals 1,000 times stronger. There are still a large number of emission features visible at 3 times the noise floor, but the spectrum is not totally confusion limited. A confusion limited spectrum will have a baseline emission floor that comes from weak molecular signals occupying every data channel, which overtakes the instrument noise floor and limits the vertical dynamic range. The solvent choice could limit the dynamic range for a gas mixture analysis. For example, the pure reference spectrum of ethyl cyanide, is confusion limited at $0.1 \%$ of the strongest signals. [31]

For applications where the sample matrix is uncatalogued, the broadband search mode is appropriate for surveying the composition before dedicated monitoring of specific analytes. The rotational spectrum is suitable for automated library matching in complex gas mixtures, whereby cataloged reference spectra are used to systematically detect all known species in the broadband spectrum. The negative spectra plotted in Fig. 3.1 are examples of reference spectra of the headspace of each component dissolved in methanol and diluted 10:1 in water. For illustration purposes the methanol contribution to each reference spectrum is subtracted out (a pure methanol headspace reference spectrum was also measured). Both the high-resolution and the spectral redundancy contribute to the simple, high confidence ability to identify components in the mixture. All of the molecules in the EPA VOC mix \#6 have a repeating spectral pattern for each quantized total angular momentum state (quantum number J) within the instrument band, which is the typical case for molecules with 3 or more, heavy atoms. For this mixture, if one examines the top $90 \%$ by intensity, of each molecule's lines it is rare that there are line overlaps. However, the high spectral redundancy practically 
ensures that there is sufficient clean spectral space to make a confident detection of each species in the mixture. It is important to note that both the frequency and intensity pattern are parameters that influence high-confidence library matching. The frequency dependent excitation source and response of the spectrometer is accounted for in a calibration scheme described elsewhere in order to achieve spectral intensity accuracy. [31]

Also visible in the full band view are repeated patterns of a different nature for the chlorinated and brominated species. Chlorine and bromine each have two isotopes with considerable natural abundance. Since the rotational spectrum is directly influenced by a molecule's mass distribution, isotopologues give distinctly shifted spectra with relative intensities that match the relative isotope abundance. The chlorine isotopes ${ }^{35} \mathrm{Cl}$ and ${ }^{37} \mathrm{Cl}$ have a natural abundance of 3:1 and the two chloromethane patterns at the low end of the band match a 3:1 relative intensity ratio. The same is illustrated for bromomethane with approximately equal abundance between ${ }^{79} \mathrm{Br}$ and ${ }^{81} \mathrm{Br}$. The isotopologue patterns can be used to determine accurate isotopic ratios. Monitoring the chlorine isotopic ratio is a strength for rotational spectroscopy that is challenging for current GC-MS techniques which require chemical conversion of the analyte. [54]

Generally, headspace measurements involve vapor-liquid phase equilibration into atmosphere. High-resolution rotational spectroscopy requires low pressure measurements around 10 mTorr $\left(\sim 10^{-5}\right.$ atm), above which pressure broadening pushes the integrated signal intensity to the wings of the line resulting in long Lorentzian tails that degrade the baseline resolution of the measurement. Because the pressure must be reduced, the millimeter wave spectrum presented in this work is not strictly a 
measurement of the partial pressure composition, but rather the fractional pressure. Assuming an ideal gas, the equilibrium partial pressure $\left(P_{i}\right)$ of each component in the headspace is independent of the total pressure $\left(P_{t o t}\right)$. As a result, the fractional pressure $\left(f_{i, m e a s}\right)$ of each volatile species can be enhanced by removing the atmosphere before equilibration so that there is a lower total pressure. Equation 3.1 shows the relationship between Henry's Law constant $\left(k_{h, i}\right)$ and partial pressure. The rotational spectrum is measured by reducing the total pressure to 10 mTorr (equation 3.2) so the partial pressure of component $i$ is enhanced if there is less total pressure in the headspace (equation 3.3). Within experimental error, the fractional pressure of chloromethane in a $20 \mu \mathrm{g} / \mathrm{mL}$ solution headspace expansion into vacuum is measured to be 16 times higher than for expansion into atmosphere (see Table 3.2).

$$
\begin{gathered}
P_{i}=k_{h, i}[i] \\
f_{i, \text { meas }}=\frac{P_{i, \text { meas }}}{10 \mathrm{mTorr}}=\frac{k_{h}[i]}{P_{\text {tot }}} \\
P_{i, \text { meas }}=\frac{k_{h}[i]}{P_{\text {tot }}} * 10 \mathrm{mTorr}
\end{gathered}
$$

A practical method for removing the atmosphere contribution to the total pressure is to simply inject the VOC solution into an evacuated vial sealed by rubber septum, allow time for phase equilibrium, and sample the headspace through a needle connected to the spectrometer vacuum line. Needle sampling through sealed vials is the conventional method for headspace sampling (and solution sampling). With the atmosphere removed, the total pressure will represent the sum of the partial pressures of the analytes and the vapor pressure of the solvent. If all analytes are at very low mole fractions in the solution, then they will only contribute fractionally small amounts to the headspace pressure so that the total pressure is dominated by vapor pressure of the 
solvent. Since low vapor pressure solvents are favorable, water was chosen as a solvent over methanol. There are several reasons for diluting the standard methanol solution in water. The first is that Henry's Law constants are generally available for volatiles dissolved in water, but another is the fact that with a vapor pressure of 18 Torr it contributes 5 times less vapor than methanol (100 Torr) to the total pressure.

Another advantage of using water is that it contributes no appreciable rotational spectrum at room temperature in the $260-295 \mathrm{GHz}$ band of the spectrometer. This can be viewed as a significant advantage for rotational spectroscopy; water causes no spectral interference. The water solvent also makes the sample matrix compatible with the EPA method for determining volatile impurities in water and the USP method 467 for residual solvents. [55] In general, for residual solvent analysis, it is favorable to use a low vapor pressure solvent which will elute very late from the column so that it doesn't interfere with the analytes. For example, dimethylacetimide (DMAC) with a vapor pressure of 2 Torr is a common solvent allowed for in the USP method.

In addition to contributing vapor pressure, solvent choice also influences the fractionation of an analyte into the headspace since it will have different solubility in different solvents. For solutes that have poor solubility in water, methanol generally enhances the solubility and lowers the fractionation into headspace. [56-58] This effect has been measured and is generally considered not to have much of an effect so long as the methanol concentration is well below $1 \%$. The solution headspace of the original EPA VOC mix \#6 dissolved in 100\% methanol solution headspace was also measured. After diluting 20:1 in water, the signal levels did not drop, they were slightly enhanced due to the decreased solubility in water, corresponding with an increase in partial 
Table 3.2: Enhancement by vacuum equilibration ${ }^{\text {a }}$ Fractional

Pressure

\begin{tabular}{lcccc}
$\begin{array}{l}\text { Equilibration } \\
\text { Method }\end{array}$ & $\begin{array}{c}\text { Calc. } \\
(\mu \text { Torr) }\end{array}$ & $\begin{array}{c}\text { Obs. } \\
(\mu \text { Torr) }\end{array}$ & $\%$ error & factor \\
\hline vacuum & 929 & 600 & 35.4 & $\mathbf{1 6}$ \\
atmosphere & 34.0 & 37.5 & 10.4 & $\mathbf{1}$ \\
\hline
\end{tabular}

a) chloromethane $20 \mu \mathrm{g} / \mathrm{mL}$ in $10 \%$ methanol 
pressures and enhanced fractional pressures due to the lower vapor pressure contribution from water.

The dichlorodifluoromethane signal was higher by a factor of 10 and can be explained due to its high fractionation out of water (high Henry' Law Constant). Methanol has the greatest effect on altering the fractionation into the vapor phase for volatiles least soluble in water. The next least soluble component in water is trichlorofluoromethane.The 5\% (by volume) methanol concentration in the stock, $100 \mu \mathrm{g} / \mathrm{mL}$ water solution is expected to cause some deviation from measured Henry's Law behavior in water. Obtaining pure dichlorofluoromethane, vinyl chloride, and bromomethane for reference spectra were not practical. However, methanol, chloromethane, chloroethane, and trichlorofluoromethane (a high vapor pressure liquid) were all available for pure reference measurements in order to independently determine the fractional pressure. So long as the pressure is below the pressure broadening limit (< 10 mTorr), signals decrease linearly with molecule number density (or partial pressure) all of the way down to the detection limit $(<30 \mathrm{nTorr}$ for chloromethane and chloroethane). The partial pressure of a gas is determined by scaling the intensity of the pure reference spectrum to the measured spectrum and applying the scaling factor to the reference spectrum pressure. When summing the expected contribution of partial pressure of all of the analytes, the measured fractional pressure of approximately 500 mTorr of chloromethane and $450 \mathrm{mTorr}$ of chloroethane were within experimental precision (see Table 3.3). The experimental precision is determined by the reproducibility of the sampling method and is approximately $30 \%$. Back to back spectra of the same sample are reproducible to better than $1 \%$ by CP-FTmmW. [31] The 
trichlorofluoromethane, however, has a $90 \%$ error. The 5\% methanol is expected to contribute a reduction in headspace fractionation for trichlorofluoromethane and dichlorofluoromethane since they have the highest Henry's Law constants (and lowest solubility in water). Additionally, measurements of Henry's Law constants are historically accepted to have $30 \%$ error, which would affect the calculated total pressure and therefore the predicted fractional pressures. Since any introduction of air would affect the fractional pressures of the analytes, it is an important source of error and requires that the sample holder maintain a good seal against ambient air. Such was the basis for benchmarking the technique using the sampling method presented in the experimental section, which included a tightly sealed glass tube (by Swagelok on one end and Viton o-ring on the other end) and air removal after freezing the mixture.

\section{B. Targeted Mixture Survey}

At a 200:1 dilution in water, the trichlofofluoromethane is no longer resolved from the base line. In order to detect trichlorofluoromethane at lower concentrations, a 1 million shot HDR spectrum could be measured, however at two hours of measurement time further broadband signal averaging becomes prohibitive for maintaining sample integrity (and moves beyond the scope of high throughput, fast spectroscopy). Since the spectrometer is driven by an AWG, any excitation scheme (within the nyquist limits of $12 \mathrm{GS} / \mathrm{s}$ ) can be implemented. In targeted mode, a single color, transform limited excitation pulse can be utilized in order to put all of the millimeter wave source power into the narrow spectral band of interest. Additionally, the pulse length can be optimized for maximum population inversion (the $\pi / 2$ condition). 
Table 3.3: EPA VOC mix \#6 Headspace Fractionation at $100 \mu \mathrm{g} / \mathrm{mL}$

\begin{tabular}{|c|c|c|c|c|c|c|}
\hline Chemical & 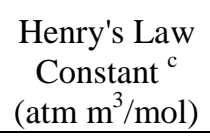 & $\begin{array}{c}\text { Conc. } \\
\left(\mathrm{mol} / \mathrm{m}^{3}\right)\end{array}$ & $\begin{array}{l}\text { Partial } \\
\text { Pressure } \\
\text { (Torr) }\end{array}$ & $\begin{array}{c}\text { Fractional } \\
\text { Pressure } \\
(\mu \text { Torr }) \\
\end{array}$ & $\begin{array}{c}\text { Observed } \\
(\mu \text { Torr })\end{array}$ & $\begin{array}{c}\% \\
\text { error }\end{array}$ \\
\hline Chloromethane $^{\text {a }}$ & $8.82 \mathrm{E}-03$ & 1.98 & 13.3 & 430 & 500 & 16.2 \\
\hline Chloroethane $^{\text {a }}$ & $1.11 \mathrm{E}-02$ & 1.55 & 13.1 & 424 & 450 & 6.2 \\
\hline Bromomethane & $6.24 \mathrm{E}-03$ & 1.05 & 5.00 & 162 & -- & -- \\
\hline Vinyl Chloride & $2.70 \mathrm{E}-02$ & 1.60 & 32.8 & 1060 & -- & -- \\
\hline Dichlorodifluoromethane & $2.25 \mathrm{E}-01$ & 0.827 & 141 & 4580 & -- & -- \\
\hline Trichlorofluoromethane ${ }^{a}$ & $9.70 \mathrm{E}-02$ & 0.728 & 53.7 & 1740 & 150 & 91.4 \\
\hline Methanol ${ }^{\mathrm{a}, \mathrm{b}}$ & $4.55 \mathrm{E}-06$ & 1230 & 30.9 & -- & 1000 & -- \\
\hline Water $^{\mathrm{b}}$ & -- & -- & 18.5 & -- & -- & -- \\
\hline
\end{tabular}

a) Pure reference spectrum measured

b) Diluent

c) See Table 3.1 for references 
Nutation curves are measured for each targeted molecular transition whereby the signal intensity is monitored as a function of the excitation pulse duration (see Fig. 3.2). The optimal $\pi / 2$-pulse is determined from the Rabi frequency $\left(\omega_{R}\right)$ which is related to the strength of the excitation pulse electric field $(E)$ and the transition dipole moment $\left(\mu_{i, j}\right)$ of the molecular transition.

$$
\omega_{R}=\frac{\mu_{10} E}{\hbar}
$$

The excitation field strength is a function of frequency in the spectrometer band and transition dipole moments will differ with each species (and molecular transition to a smaller degree), so the $\pi / 2$-pulse length should be measured for each targeted transition. For chloromethane, a $200 \mu$ s excitation pulse was optimum. In general, the $\pi / 2$ optimized excitation produced up to a factor of 2 enhancement in the signals compared to the broadband HDR measurement. The major sensitivity benefit comes from signal averaging, which is much faster because only a few spectral segments are monitored; the rest is left out. A 10 million shot targeted acquisition takes 40 seconds ( $2 \mu$ s cycles). Signal intensity versus partial pressure for chloromethane and chloroethane is shown in figures 3.3 and 3.4, respectively. Linearity is established across 6 orders of magnitude in dynamic range. In targeted mode, the total time is cumulative for each consecutive transition monitored. Although the signal averaging is a round robin scheme of addressing a new transition every $2 \mu \mathrm{s}$. The targeted detection sensitivities for chloromethane, chloroethane, bromomethane, and vinyl chloride are all less than $1 \mu \mathrm{g} / \mathrm{L}$ (see Table 3.4) showing matched performance to the EPA method 524 which entails preconcentration followed by GC-MS, one of the most sensitive techniques for trace detection of volatiles. 


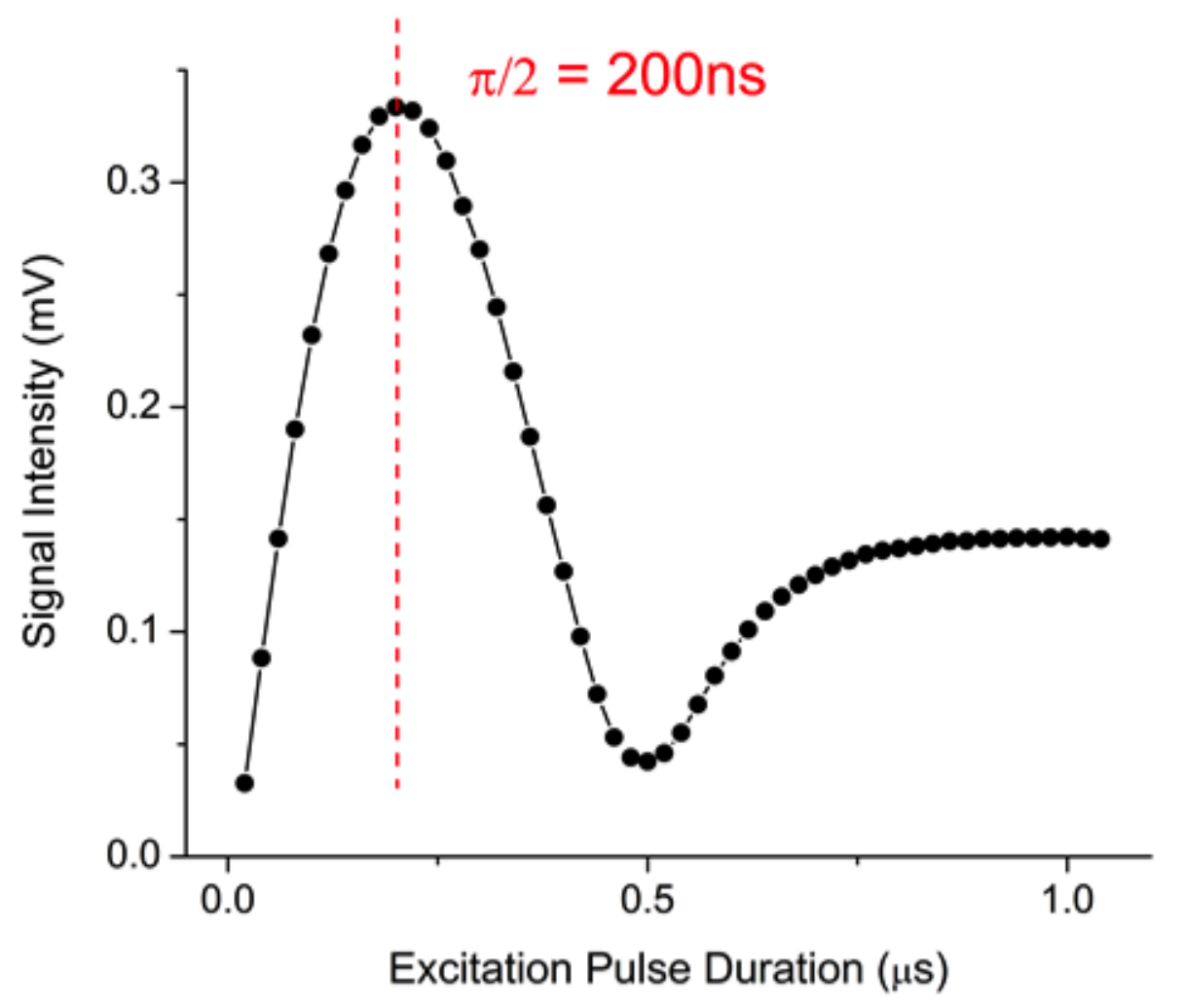

Figure 3.2: A nutation curve for the $292346.9 \mathrm{GHz}$ transition of chloromethane. The excitation pulse drives the population inversion of two adjacent rotational energy levels at the Rabi frequency where maximum population inversion occurs at $\pi / 2$. 


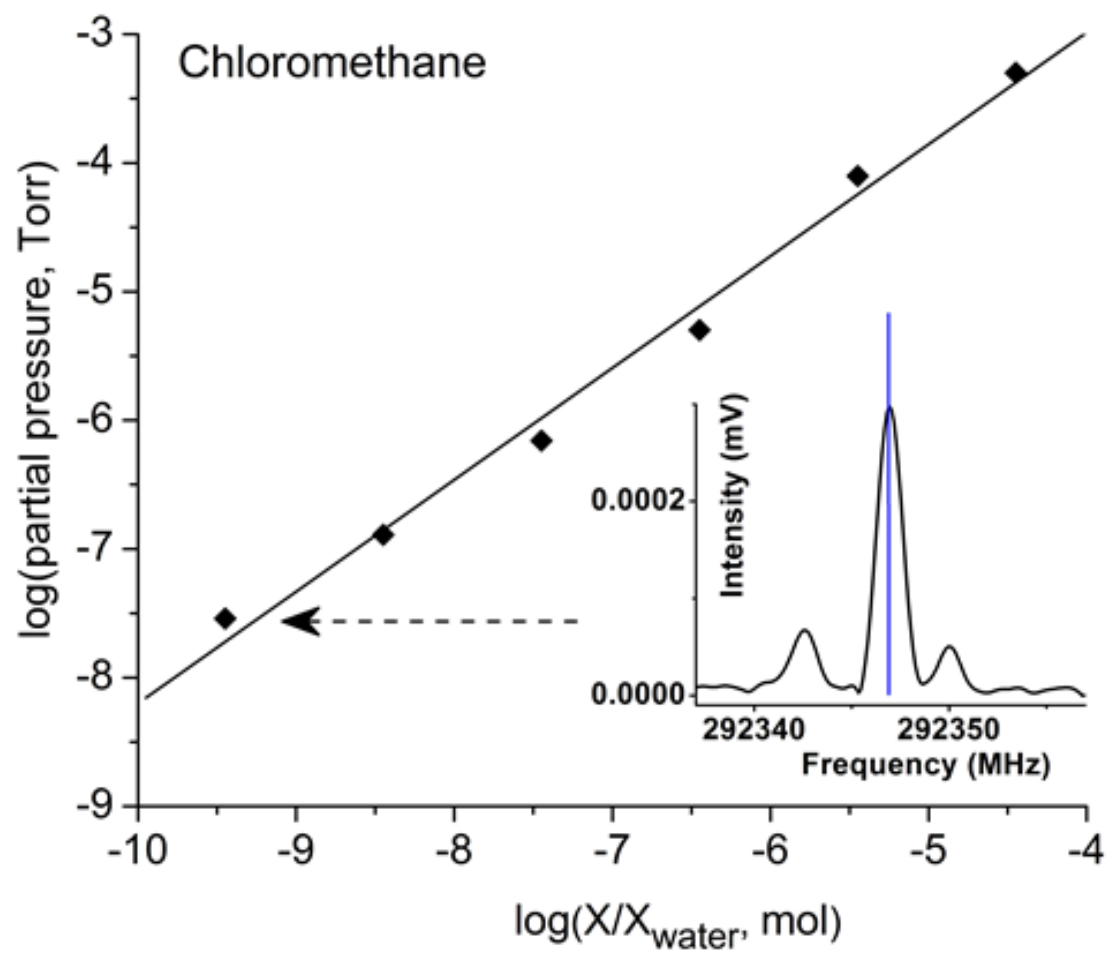

Figure 3.3: Logarithmic response curve for chloromethane measured in the mixture by serial dilutions. The inset illustrates the signal-to-noise at $1 \mu \mathrm{g} / \mathrm{L}$. 


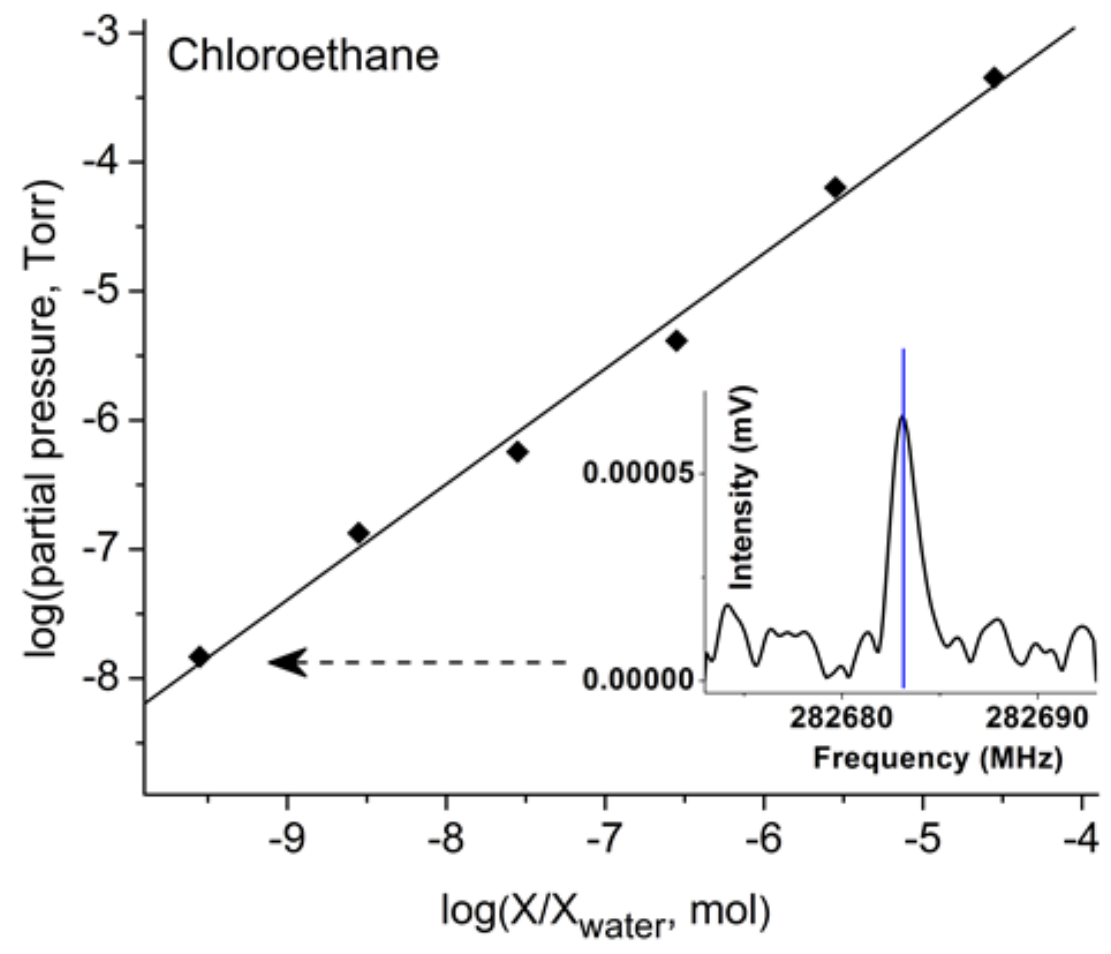

Figure 3.4: Logarithmic response curve for chloroethane measured in the mixture by serial dilutions. The inset illustrates the signal-to-noise at $1 \mu \mathrm{g} / \mathrm{L}$. 
Although the targeted mode is not an unbiased, full composition analysis, it is the mode of choice for situations where only a few analytes are of importance. For example in a manufacturing setting regulated by the FDA, residual solvents need only be quantified when they are part of the synthesis. It is undesirable to spend analysis time on results that do not push the product to release. There is no capability to trade time for analysis breadth in the EPA method 524. For chromatography, resolution can be traded for speed, and custom temperature control can selectively resolve analytes of interest, but it requires customization of the measurement and is not a universal mode. Changing the CP-FTmmW broadband analysis to a targeted analysis does not require any change in sampling or equipment. With the flexibility of the CP-FTmmW spectrometer, there is full control over the balance of analysis depth and measurement time via selection of desired signal averaging depth and frequency band.

\section{Discussion and Conclusions}

It is important to note how the sample matrix affects the instrument data sheet specifications for detection limit and detection confidence. Detection limits for many techniques are highly matrix dependent and may be misrepresented when determined from pure standards. The headspace analysis of EPA VOC mix \#6 keeps true to the goal of evaluating CP-FTmmW performance on a real mixture that is relevant to the chemical industry. The rotational spectrum of a chemical used for library reference spectra will be made up of contributions from isotopologues of the main species in natural relative abundance, molecules in vibrationally excited states, and low barrier conformations or 
Table 3.4: Detection Limits in Water

\begin{tabular}{ccc}
$\left.\frac{\text { Broadband }}{(5 \mathrm{~min}, \mu \mathrm{g} / \mathrm{L}}\right)$ & $\frac{\text { Target Mix }}{(10 \mathrm{~min}, \mu \mathrm{g} / \mathrm{L})}$ & $60 \underline{\text { EPA 524 }}$ \\
5 & 0.1 & 0.1 \\
22 & 0.8 & 0.1 \\
26 & 0.5 & 0.1 \\
42 & 0.5 & 0.2 \\
100,000 & 1000 & 0.1 \\
100,000 & 1000 & 0.1 \\
\hline
\end{tabular}

Chloromethane

Bromomethane

Chloroethane

Vinyl Chloride

Dichlorodifluoromethane

100,000

1000 
isomers. Capturing all of the emission requires the high dynamic range capabilities of CP-FTmmW. Typically, calibration curves are measured within the detection range of interest. Since the CP-FTmmW spectrum is capable of measuring 6 orders of magnitude of dynamic range in minutes, instrument verification measurements can be measured at high concentrations where samples can be easily prepared. For example, reference library spectra can be recorded from pure, 1 mTorr standards.

The full analytical chemistry instrument suite is made up of many niche instruments. There is no universal detector, but broad chemical coverage is an important characteristic to maximize because it is a major influence to commercial potential. Universal platforms lead to low manufacturing costs, broad market applicability, and high return on investment to the end user. The chemical niche for rotational spectroscopy has a rigid requirement that the molecule be polar. Beyond that, the analytical space for rotational spectroscopy is large. The strength of CP-FTmmW is for sensitive room temperature detection of low molecular weight, polar volatiles $(<150 \mathrm{amu})$ and is the basis for choosing the $260-295 \mathrm{GHz}$ band for the first design. However, the platform design for the CP-FTmmW $260-295 \mathrm{GHz}$ spectrometer can be applied to other frequency ranges. Broadband sources (> $30 \mathrm{GHz}$ ) are commercially available from $75 \mathrm{GHz}$ up to $2 \mathrm{THz}$. [59] Halogenated species (like refrigerants) tend to have higher molecular weights (towards $150 \mathrm{amu}$ and above). Peak spectral intensity for these kinds of molecules favors frequency bands below $260 \mathrm{GHz}$. A large molecule detector (for detecting trichlorofluoromethane and dichlorodifluoromethane) might make use of $75-110 \mathrm{GHz}$ sources. On the other extreme, low molecular weight molecules (diatomics or molecules with only two heavy atoms, like methanol) favor frequency 
bands above $295 \mathrm{GHz}$. Chirped pulse measurements have been extended up to $860 \mathrm{GHz}$ where the spectrum of methanol was measured for the performance demonstration. [28] At some point (above $500 \mathrm{amu}$ ), the complexity of the rotational spectrum becomes prohibitive and the volatility of the chemical reduced so that gas mixture analysis is no longer applicable. The $260-295 \mathrm{GHz}$ spectrometer is ideally suited for composition analysis addressing common solvents used in chemical synthesis. They are typically of low mass and are polar.

CP-FTmmW spectrometers match the absolute recognition capability of rotational spectroscopy with high-performance, automated technology for direct gas mixture analysis. In order to displace current techniques, new technology must offer entirely new capabilities or offer a substantial improvement over existing techniques. Considering the broadband speed enhancements that CP-FTmmW brings to the current state of millimeter spectroscopy, trace composition analysis can be achieved in minutes, a time scale 10 times faster than typical EPA VOC methods. The comparison of CP-FTmmW direct headspace analysis of EPA VOC mix \#6 to EPA method 524 indicate competitive performance and introduce a highly simplified technique that offers automated, high throughput capabilities for the analysis of volatiles without any consumables. Methods for headspace enhancements (heating, salt addition) and for direct injection can also be incorporated for CP-FTmmW. [60, 61]

The cost curve for high frequency components driven by the defense and communications industry will continue drop, and performance will continue to increase. Even without the mass production efficiency that will come with integrated circuits and chips, the cost is already appropriate for industry adoption, and the performance 
demonstrated on the VOC mix \#6 draws attention to the analytical power for gas mixture analysis. The CP-FTmmW spectrometer is a strong design for transforming rotational spectroscopy into a tool for tackling industry scale analytical challenges and creating the market drive for scaled up manufacturing of an instrument product line.

\section{References for Chapter 3:}

1. R. D. Snook, Analyst, 2000, 125, 45.

2. I. R. Medvedev, M. Behnke, F. C. De Lucia, Analyst, 2006, 131, 1299.

3. C. Townes and A. Schawlow, Microwave Spectroscopy, Dover, New York, 1975.

4. W. Gordy and R.L. Cook, Microwave Molecular Spectra, Wiley-Interscience, 1984.

5. J. K. G. Watson, A. Roytburg, W. Ulrich, J. Mol. Spec., 1999, 196, 102.

6. H. W. Harrington, J. R. Hearn, R. F. Rauskolb, Hewlett-Packard Journal, 1971, $22,1$.

7. R. H. Hughes, E. B. Wilson, Phys. Rev., 1947, 71, 562.

8. S. Armstrong, Applied Spectroscopy, 1969, 23, 575.

9. T. J. Balle, W. H. Flygare, 1981, Rev. Sci. Intsr., 53, 33.

10. E. J. Campbell, L. W. Buxton, T. J. Balle, M. R. Keenan, W. H. Flygare, 1981, J. Chem. Phys., 74, 829.

11. E. J. Campbell, L. W. Buxton, T. J. Balle, W. H. Flygare, 1981, J. Chem. Phys., 74, 813.

12. R. D. Suenram, J. U. Grabow, A. Zuban, I. Leanov, Rev. Sci. Instru., 1999, 70, 2127.

13. M. D. Harmony, K. A. Beran, D. M. Angst, K. L. Ratzlaff, Rev. Sci. Instru., 1995, 66, 5196.

14. D. Petkie, T. Goyette, R. Bettens, S. Belov, S. Belov, S. Albert, P. Helminger, F. De Lucia, Rev. Sci. Instr., 1997, 68, 1675.

15. I. R. Medvedev, C. F. Neese, G. M. Plummer, F. C. De Lucia, Opt. Lett., 2010, 35, 1533. 
16. C. Neese, I. Medvedev, G. Plummer, A. Frank, C. Ball, F. De Lucia, IEEE Sensors J., 2012, 12, 2565.

17. S. Albert, D.T. Petkie, R. P.A. Bettens, S.P. Belov, F. C. De Lucia, Anal. Chem. 1998, 70, 719A.

18. F. C. De Lucia, J. Opt. Soc. Am. B, 2004, 21, 1273.

19. R. R. Ernst, W. A. Anderson, Rev. Sci. Instru., 1966, 37, 93.

20. G. G. Brown, B. C. Dian, K. O. Douglass, S. M. Geyer, B. H. Pate, J. Mol. Spec., 2006, 238, 200.

21. G. G. Brown, B. C. Dian, K. O. Douglass, S. M. Geyer, S. T. Shipman, B. H. Pate, Rev. Sci. Instr., 2008, 79, 053103.

22. D. Zaleski, J. Neill, M. Muckle, N. Seifert, P. Carroll, S. WidicusWeaver, B. Pate, J. Mol. Spec., 2012, 280, 68.

23. G.S. Grubbs, C. T. Dewberry, K. C. Etchison, K. E. Kerr, S. A. Cooke, Rev. Sci. Instr., 2007, 78, 096106.

24. S. L. Stevens, N. R. Walker J. Mol. Spec., 2010, 263, 27.

25. D. A. Obenchain, A. A. Elliott, A. L. Steber, R. A. Peebles, S. A. Peebles, C. J. Wurrey, G. A. Guirgis, J. Mol. Spec., 2010, 261, 35.

26. R. G. Bird, J. L. Neill, V. J. Alstadt, J. W. Young, B. H. Pate, D.W. Pratt, J. Phys. Chem. A, 2011, 115, 9392.

27. A. L. Steber, B. J. Harris, J. L. Neill, B. H. Pate, J. Mol. Spec., 2012, $280,3$.

28. J. L. Niell, B. J. Harris, A. L. Steber, K. O. Douglas, D. F. Plusquellic, B. H. Pate, Opt. Expres., 2013, 21, 19743.

29. E. Gerecht, K. Douglass, D. Plusquellic, Opt. Lett., 2011, 19, 8973.

30. G. B. Park, A. H. Steeves, K. Kuyanov-Prozument, J. L. Neill, R. W. Field, J. Chem. Phys., 2011, 135, 024202.

31. B. J. Harris, A. L. Steber, B. H. Pate, Rev. Sci. Instr., in preparation.

32. S. M. Fortman, I. Medvedev, C. Neese, F. C. De Lucia, 2010, ApJ, 725, 1682. 
33. U.S. Food and Drug Administration, Guidance for Industry, PAT-A Framework for Innovative Pharmaceutical Development, Manufacturing, and Quality Assurance, September 2004.

34. D. C. Hinz, Anal. Bioanal. Chem., 2006, 384, 1036.

35. USEPA, Measurement of Purgeable Organic Compounds in Water by Capillary Column Gas Chromatography/Mass Spectrometery, Method 524.2, Environmental Monitoring Systems Laboratory, Cincinnati, OH, USA, 1982.

36. USEPA. Code of Federal Regulations: Title 40 part 136, Appendix B. Methods for Organic Chemical Analysis of Municipal and Industrial Wastewater, Method 624 Purgeables, Office of Science and Technology Engineering and Analysis Division, Washington D. C., USA, 2013.

37. USEPA. Determination of Volatile Organic Compounds in Ambient Air Using Specially Prepared Canisters with Subsequent Analysis by Gas Chromotagraphy, Compendium Method TO-14A, Center for Environmental Research Information, Cincinnati, OH, USA, 1999.

38. USEPA. Determination of Volatile Organic Compounds in Air Collected in SpeciallyPrepared Canisters and Analyzed by Gas Chromotagraphy/Mass Spectrometry, Compendium Method TO-15, Center for Environmental Research Information, Cincinnati, OH, USA, 1999.

39.USEPA. Determination of Volatile Organic Compounds in Ambient Air Using Active Sampling onto Sorbent Tubes, Compendium Method TO-17, Center for Environmental Research Information, Cincinnati, OH, USA, 1999.

40. USEPA. SW-846: Test Methods for Evaluating Solid Waste, Physical/Chemical Methods, Method 8260B, Office of Solid Waste, Washington D. C., USA, 1996.

41. USEPA. SW-846: Test Methods for Evaluating Solid Waste, Physical/Chemical Methods, Method 5035, Office of Solid Waste, Washington D. C., USA, 1996.

42. D. R. Knapp. Handbook of analytical derivatization reactions, Wiley, 1979.

43. Y. Roggo, P. Chalus, L. Maurer, C. Lema-Martinez, A. Edmond, N. Jent, 2007, J. Pharm. Bio. Anal., 44, 683.

44. S. Schreyer, Eastern Analytical Symposium, Sommerset, NJ, 2013, Abstract 458.

45. E. R Crosson, 2008, Appl. Phys., 92, 403.

46. K. K. Lehmann, 1996, U. S. Patent 5528040. 
47. F. K. Tittel, R. Lewicki, R. Lascola, S. McWhorter in, Trace Analysis of Specialty and Electronic Gases, ed. W. M Geiger and M. W. Raynor, Wiley, New Jersey, 2013, ch. 4.

48. B. M. Marquez, D. Foley, IFPAC Process Analytical Chemistry, Washington D. C., 2014, Abstract 347.

49. S. W. Smith, The Scientist and Engineer's Guide to Digital Signal Processing, California Technical Publishing, San Diego, 1997.

50. J. M. Gosset, Environ. Sci. Technol., 1987, 21, 202.

51. USEPA. Air and steam stripping of toxic pollutants. Tech. Rep. EPA-68-03-002, Industrial Environmental Research Laboratory, Cincinnati, OH, USA, 1982.

52. R. D. Nelson Jr., D. R. Lide, A. A. Maryott, Selected Values of electric dipole moments for molecules in the gas phase, NSRDS-NBS10, 1967.

53. C. G. Gray, K. E. Gubbins, Theory of molecular fluids. Volume 1: Fundamentals, Clarendon Press, Oxford 1984.

54. T. Kuder, P. Philp, Environ. Sci. Technol., 2013, 47, 1461.

55. USP-NF. General Chapter 467: Residual Solvents, 2007.

56. C. Munz, P. V. Roberts, J. Am. Water. Works Assoc., 1987, 79, 62.

57. C. T. Chiou, R. L. Malcolm, T. I. Brinton, D. E. Kile, 1986, 20, 502.

58. J. Staudinger, P. V. Roberts, Critical Rev. in Environ. Sci. and Tech., 1996, 26, 205.

59. J. C. Pearson, B. J. Drouin, A. Maestrini, I. Mehdi, J. Ward, R H. Lin, S. Yu, J. J. Gill, B. Thomas, C. Lee, G. Chattopadhyay, E. Schlecht, F. W. Maiwald, P. F. Goldsmith, P. Siegel, 2011, Rev. Sci. Instru., 82, 093105.

60. J. Takeoka, W. Jennings, J. Chromatogr. Sci.,1984, 22, 177.

61. S. L. Friant, I. H. Suffet, Analytical Chemistry, 1979,51, 2167. 


\section{Chapter 4. Mixture Analysis - Solids}

The millimeter-wave rotational spectrum of the 4(5)-methylimidazole tautomeric pair

\section{Introduction}

Nucleic acid bases (NABs), the building blocks of DNA, are well known to exhibit many conformers with appreciable abundance in thermal equilibrium. Structural studies of NAB tautomers have been driven by their suggested role in the mutagenesis of DNA. [1-4] Computational methods for predicting spectroscopic parameters of NAB tautomers are usually paired with experiment in order to address the challenge of independently characterizing several molecular conformers in a mixture. Isolated NABs can be studied in the gas phase, independent from solvent effects using isomer selective spectroscopy in the UV/IR and microwave region of the spectrum. [6-9, 10-15] UV/IR methods usually involve double resonance techniques to achieve selectivity whereas the inherent structural selectivity of microwave rotational spectroscopy requires no special excitation schemes. Both techniques involve creating a vapor through melting or laser desorption of a solid sample or simulating a vapor-like environment using matrix isolation.

The NAB tautomerization happens through a proton (or double proton) transfer across conjugated rings (see Fig 4.1). From a chemist's point of view, the tautomer equilibrium is of interest in a broad biological context and provides a subject for theoretical/experimental pairing for improving computational capabilities. One class of molecules that have received much attention is imidazole derivatives. $[13,16]$ Imidazole 


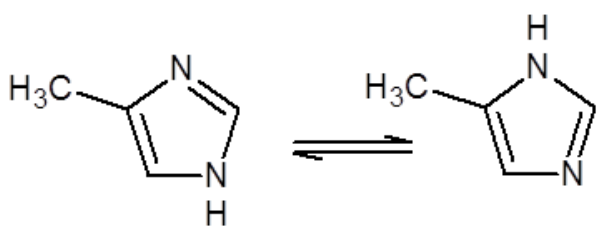

Figure 4.1: 4(5)-methylimidazole tautomerziation via proton tunneling across the two nitrogen positions on the imidazole ring. 
is the basic ring component of histamine, an important molecule involved in immune response, neurotransmission, and digestive regulation. Typical biological derivatives of imidazole involve substitution at the 4 position of the ring which generate a 4,5 tautomer from the proton transfer between the two nitrogen atoms. The microwave spectrum of imidazole has been measured and the 4-vinylimidazole tautomers have also been studied by microwave spectroscopy. [17]

Recently, toxicity studies for 4-methylimidazole have highlighted its potential carcinogenicity and raised concerns for regulating its content in foods and drinks. [18-21] During caramelization processes that use the Maillard reaction system, substituted imidazoles form as side products, including 4-methylimidazole, which has been identified at levels up to 200 ppm. [22-28] The Office of Environmental Health Hazard Assessment within the California EPA has listed 4-methylimidazole as a carcinogen and imposes a daily ingestion limit of $16 \mu \mathrm{g} / \mathrm{day}$. [29] There have been some experimental studies of the 4(5)-methylimidazole equilibrium, but the research focus has shifted from structural characterization to detection. [30,31]

Small structural differences in molecules often imply significantly different biological effects and detection can pose a challenge for techniques that are not sensitive to different isomeric forms. Although long promised through the decades as a technique with superior advantages in molecular recognition capability, with the exception of an isolated commercial design introduced in the 1970s, rotational spectroscopy has only in modern years been trending towards instrument designs suitable for analytical chemistry. [32-40] The Stark modulated microwave spectrometers conventionally used for studying tautomeric systems are not suitable for benchtop analytics. With the introduction of a 
new instrument for chirped pulse millimeter wave spectroscopy (CP-FTmmW), rotational spectroscopy finally meets the right balance of cost, performance, and simplicity for wide spread analytical chemistry challenges. [40] Microwave spectroscopy already has the hallmark of being suitable for monitoring "delicate equilibria" of isomerization, essentially an inseparable mixture. [13] The drawbacks of the older techniques are the inefficient use of sample, requiring molten materials to generate appreciable vapor pressure. CP-FTmmW is a room temperature, fast, broadband method more suitable for measuring headspace of solids because of the low sample requirements. Although analytical studies may predominantly involve liquid samples, the 4(5)-methylimidazole solid is used here to generate a reference spectrum for identification through the millimeter wave rotational spectrum. No previous rotational spectroscopy measurements have been reported in the spectroscopic literature for 4(5)-methylimidazole.

\section{Experimental}

\section{A. Spectrometer}

The 260 - 295 GHz segmented chirped pulse Fourier transform millimeter wave (CP-FTmmW) spectrometer used for the measurement of 4(5)-methylimidazole is described elsewhere. [40] To summarize here, the spectrometer is driven by a dual channel, 12 GS/s arbitrary waveform generator (AWG), which is used to generate a series of chirped pulses out of channel one with paired local oscillator (LO) sine waves out of the second channel for the heterodyne receiver. The AWG output runs from approximately from $2-4 \mathrm{GHz}$. Both the chirped pulses and local oscillator outputs are

upconverted off of a common $8.8 \mathrm{GHz}$ PDRO in order to generate the $10.7-12.2 \mathrm{GHz}$ input for solid state active multiplier chains (AMCs). The excitation source is a Virginia 
Diodes high power $\mathrm{x} 24 \mathrm{AMC}$ and the receiver is comprised of a x12 AMC which feeds the LO into a subharmonic mixer.

In the high dynamic range mode used in this study, $720 \mathrm{MHz}$ of bandwidth is addressed in 30, $100 \mathrm{~ns}, 24 \mathrm{MHz}$ chirps each followed by $1900 \mathrm{~ns}$ of detection against the same local oscillator frequency such that the intermediate down conversion frequency out of the heterodyne receiver runs from $720-1440 \mathrm{MHz}$. The entire spectrometer band is addressed after stepping up the routine by $720 \mathrm{MHz} 50$ times. After an excitation pulse, the broadband molecular free induction decay (FID) is coupled into the subharmonic mixer of the heterodyne receiver, downconverted, and digitized on an interleaved 4GS/s PCI digitizer equipped with a field programmable gate array (FPGA) for real-time signal averaging. The accumulator has a memory depth of $512 \mathrm{kpts}$ when interleaved and allows $131 \mu$ s of real-time signal accumulation (or $1440 \mathrm{MHz}$ of bandwidth in HDR mode). Each segment is fast Fourier transformed and concatenated to build the full band magnitude transform spectrum. Signal processing includes Kaiser-Bessel windowing in order to suppress Lorentzian broadening of the spectrum and enhance the dynamic range.

The spectrometer sample cell is a $65 \mathrm{~cm}$ steel tube approximately $3.8 \mathrm{~cm}$ in diameter and $1 \mathrm{~L}$ in volume. PTFE focal lenses act as windows in assist in beam collimation. The low pressure millimeter wave measurements are maintained with a compact turbo/mechanical pump combination.

\section{B. Sample}

Since pure 4(5)-methylimidazole solid is available as a stock chemical (Aldrich 199885), no synthesis is required to measure the reference spectrum. In contrast to the 
NABs, the spectrum 4(5)-methylimidazole is not complicated by the abundance of multiple conformers; however, it does include a methyl rotor complicating the analysis and requiring a fitting program that incorporates a torsional Hamiltonian. The room temperature vapor pressure of 4(5)-methylimidazole is estimated to be 7 mTorr and is sufficient for measurement by millimeter wave spectroscopy where pure spectra are recorded below a pressure broadened limit ( $<10 \mathrm{mTorr})$. [41]

The 4(5) methylimidazole powder was purified by heating and melting to release water and other volatiles. Without purification, the spectrum of 4(5) methylimidazole was dominated and totally obscured by acetic acid, which may be a residue from its chemical synthesis. After cooling, the recrystallized solid was crushed in a crucible and transferred to a steel bomb sealed by conflat and equipped with a transfer valve. After removing the air, the sample was allowed to sublime and equilibrate at room temperature for 30 minutes. Then, the transfer valve was opened slightly to set-up a slow, steady flow at approximately 5 mTorr. The flow cell was set up so to minimize solid condensate in the transfer lines. A three minute, 10,000 shot HDR spectrum was acquired.

\section{Spectral Assignment}

The rotational constants and principle axis dipole moments for 4-methylimidazole and 5-methylimidazole were calculated from a geometry optimization using Gaussian 09. [42] The calculations were performed at the m062x level with a 6-311++g(d,p) basis set including anharmonic corrections. The calculated rotational pattern for the ground vibrational state of 4-methylimidazole was matched to the measured spectrum by scaling the rotational constants using the JB95 fitting program which provides a convenient local 
fitting approach for $a v_{t}=0$, A state. [43] With an estimate of the quartic distortion constants determined using JB95 there was a good match for transitions with up to $\mathrm{K}_{\mathrm{a}}=9$. SPFIT, in combination with AABS was used to incorporate higher order distortion constants and fit transitions with quantum numbers up to $K_{a}=15$. [44,45] Only the A state converged to a suitable rms. The A/E doubling was clearly distinguished at low $\mathrm{K}_{\mathrm{a}}$ quantum numbers so that RAM36 could be used to assign the $\mathrm{A} / \mathrm{E} \mathrm{v}_{\mathrm{t}}=0$ torsion lines. [46] The initial guess parameters were taken from the SPFIT A-state constants. For 5-methylimidazole, the initial assignment in JB95 was initiated from a simulation of the spectrum using scaled computational constants, where the scale factor was determined by comparing the calculated constants for 4-methylimidazole to the fitted constants.

Since only high $J$ transitions are available for the fit, correlation between centrifugal distortion and rotation-torsion poses a challenge to determining F, rho, and $\mathrm{V}_{3}$. However, the strong lines of the spectrum can be fit to an $\mathrm{A} / \mathrm{E}$ splitting pattern in order to generate a suitable line list for molecule identification. $\mathrm{V}_{3}$ was estimated using Gaussian09 to be approximately $306.1 \mathrm{~cm}^{-1}$ and $364.2 \mathrm{~cm}^{-1}$ for 4- and 5-methylimidazole, respectively. These values served as the guess parameters in the RAM36 fit. The internal rotation constant $(\mathrm{F})$ was fixed to a value of $5.38 \mathrm{~cm}^{-1}$, calculated from the $\mathrm{sp}^{3}$ hybridized methyl rotor rotational inertia. The final optimization used fixed estimates for the $\mathrm{F}$ value while floating all other parameters. 


\section{Results}

The broadband, HDR mode, 10000 shot spectrum of 4(5)-methylimidazole is shown in Figure 4.2 at a total pressure of 5 mTorr. Across $35 \mathrm{GHz}$, there are seven harmonic patterns that generate a spread in $J$ from $J=41$ to $J=56$. Due to the strong dipole moments across the a and b principle axes for both the 4 and 5 substituted isomers of methylimidazole, the strongest lines in each spectrum result from collapsed quartets of degenerate ${ }^{\mathrm{a}} \mathrm{R}$ pairs and ${ }^{\mathrm{b}} \mathrm{R}$ pairs at low $K_{\mathrm{a}}$. The quartets become resolved at $\mathrm{K}_{\mathrm{a}}=7,8$. An additional splitting arises from the internal methyl rotor. With an intermediate barrier

height $\left(300-400 \mathrm{~cm}^{-1}\right)$ the $\mathrm{A} / \mathrm{E}$ splitting for each of the isomers are on the order of several $\mathrm{MHz}$ which appear in the spectra as recognizable doublets that separate as the $K_{\mathrm{a}}$ quantum number increases (see Fig. 4.2). The two isomer patterns are well resolved from each other and from the baseline; the 4-methylimidazole spectrum has a signal-to-noise ratio greater than 1,000 (see Fig. 4.3,4.4). At $0.1 \%$ intensity of the strongest lines, the spectrum for the mixture is very dense and on the edge of confusion limited.

The rho axis method is generally favored for fitting torsion spectra because of the ability to eliminate the Coriolis-like terms used in the principal axis method and effectively separate the torsional Hamiltonian from the rotational Hamiltonian. There is a progression in recent literature towards use of the RAM36 spectral fitting program over other options for assigning 3-fold and 6-fold internal rotation spectra in part because it is designed to handle higher total angular momentum. [46,47] 


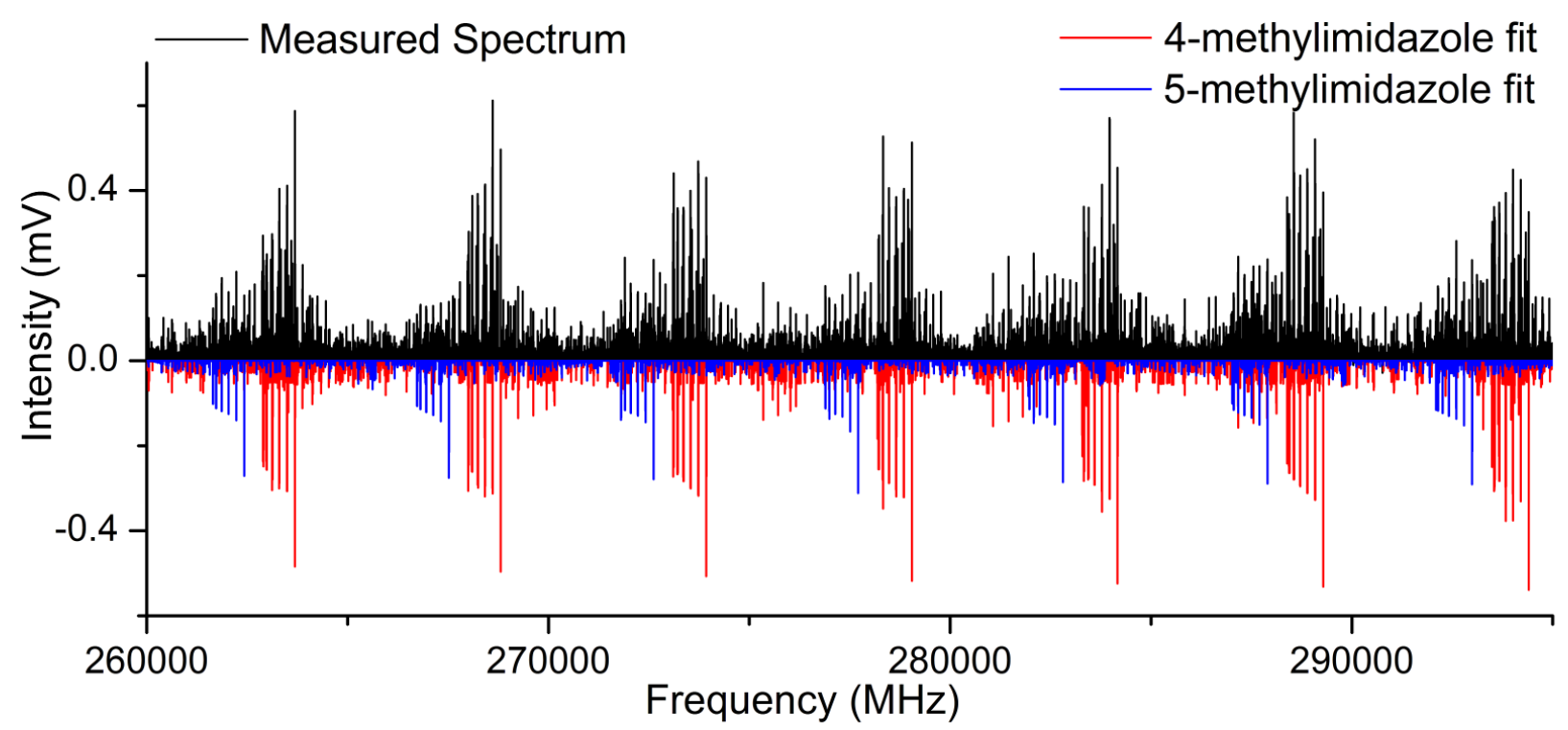

Figure 4.2: A full band, 10,000 shot, high dynamic range mode CP-FTmmW spectrum of 4(5) methylimidazole solid headspace. The total pressure is 5 mTorr with an approximately $1 \mathrm{~L} / \mathrm{min}$ steady flow. 

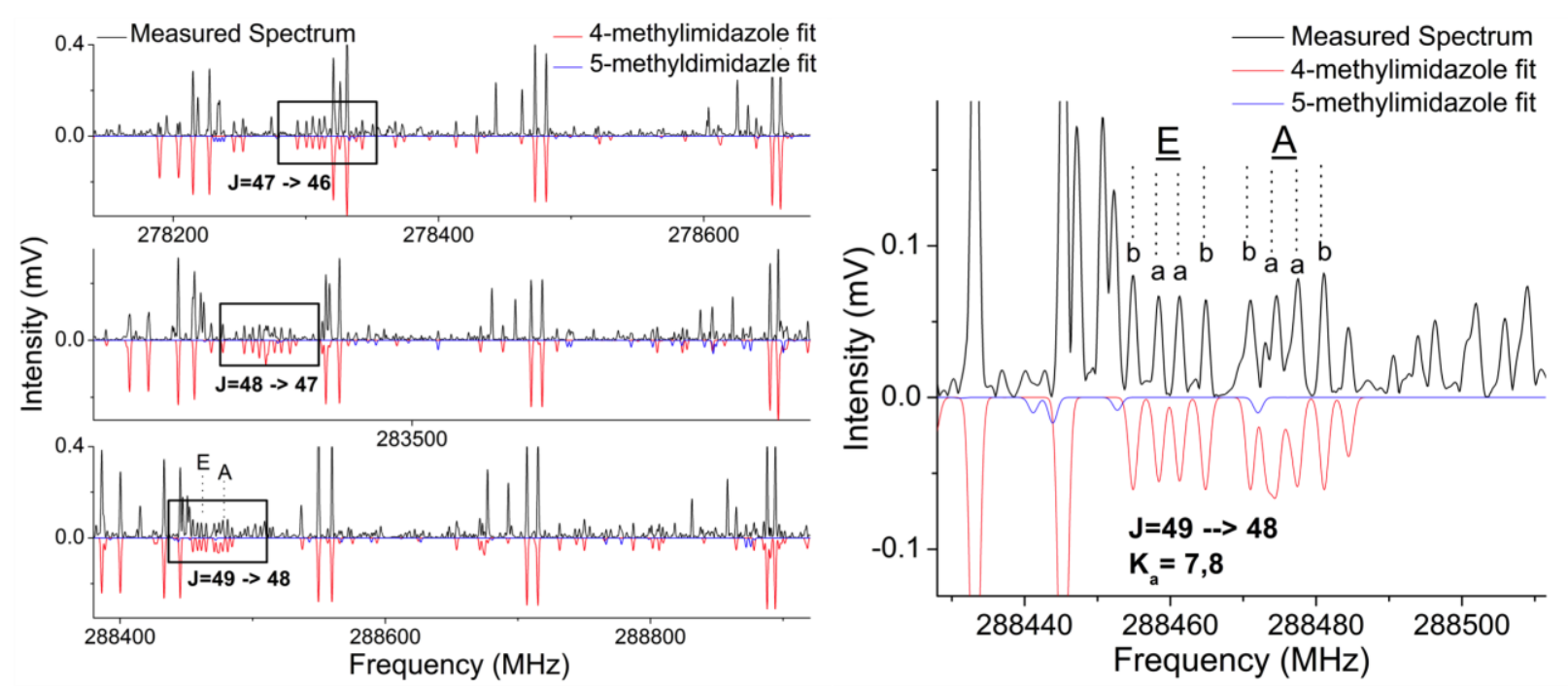

Figure 4.3: Three harmonic patterns of 4 methylimidazole. The ${ }^{a} \mathrm{R}$ and ${ }^{\mathrm{b}} \mathrm{R}$ quartets resolve out at $\mathrm{K}_{\mathrm{a}}=7,8$ shown in the black box across three harmonic patterns. The A/E splitting gives rise to 8 resolved signals. 

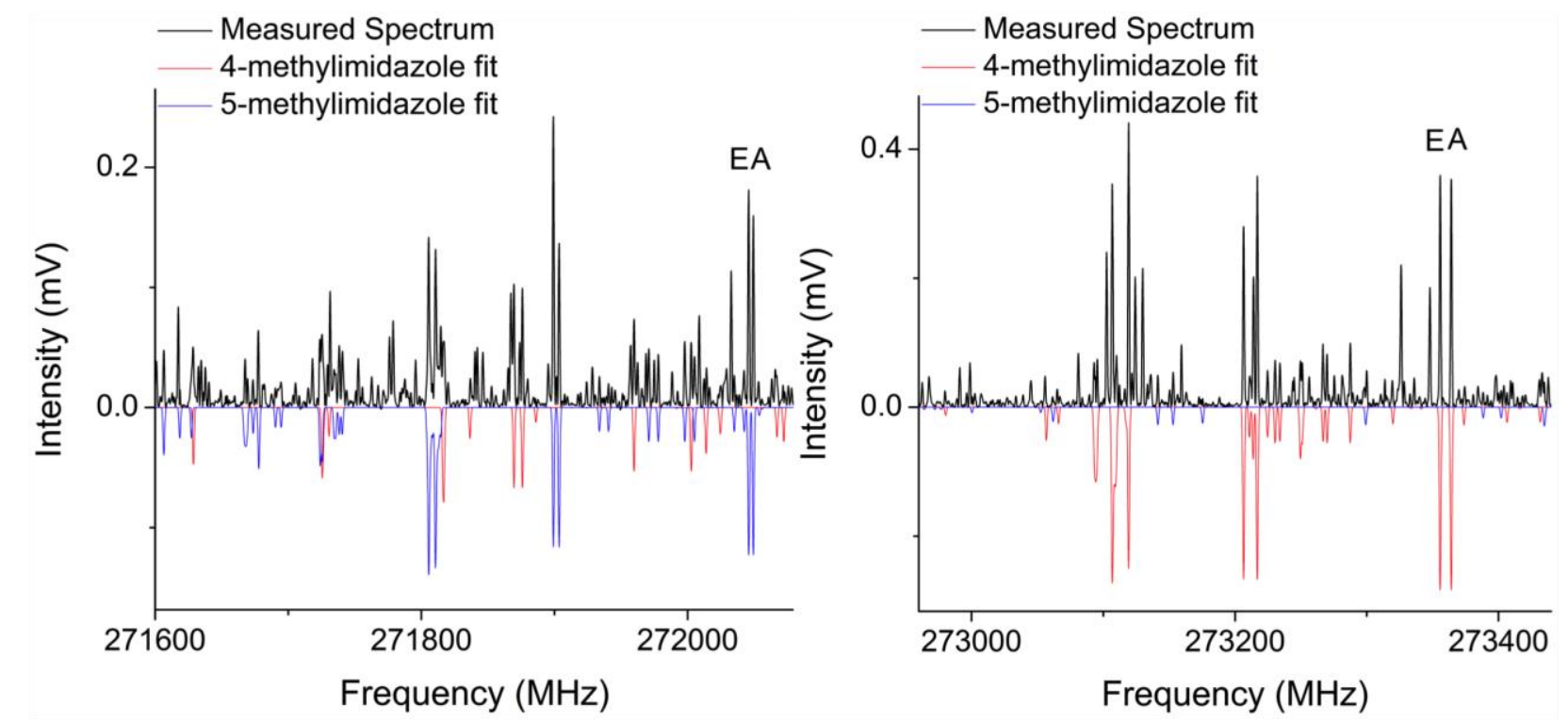

Figure 4.4: A/E splitting and isolation from 5-methylimidazole. 
It has been applied successfully for the high $J$, high $K$, global assignment of the spectrum of acetic acid. [47] Due to the high angular momentum states $(J \leq 56)$ that are probed in the 260 - $295 \mathrm{GHz}$ region for 4(5)-methylimidazole, the RAM36 program was chosen to assign the ground vibration $\mathrm{A} / \mathrm{E}$ spectral lines.

Rotational constants for 4- and 5-methylimidazole are given in Tables 4.1 and 4.2, respectively. A fit to 502 lines of 4-methylimidazole using RAM36 resulted in an rms of $82 \mathrm{kHz}$ for the A-state and $56 \mathrm{kHz}$ for the E-state. 4-methylimidazole was simulated from the fitted parameters and scaled in intensity to match the measurement. For 5-methylimidazole, 301 lines were used in the RAM36 fit giving an rms of $83 \mathrm{kHz}$ and $75 \mathrm{kHz}$ for the A and E states, respectively. The scaling factors for the rotational constants of both isomers are shown in Table 4.3, with calculated vs. SPCAT fitted values for the a-state agreeing to within $0.1 \%$ for the principal rotational constants $\mathrm{A}, \mathrm{B}$, and $\mathrm{C}$. The $\mathrm{J}$ dependent quartic distortion constants agree within $5 \%$ and the $\mathrm{K}$ dependent constants, $\Delta_{\mathrm{K}}$ and $\delta_{\mathrm{K}}$, have larger discrepancy of $80 \%$ and $30 \%$ respectively. The M062x calculation of the $\mathrm{V}_{3}$ internal rotation barriers of $306.1 \mathrm{~cm}^{-1}$ and $364.2 \mathrm{~cm}^{-1}$ for 4- and 5-methylimidazole respectively, agreed with experiment to better than $4 \%$. Using the global A/E fit from RAM36, the experimental value of $V_{3}$ is $311.9 \mathrm{~cm}^{-1}$ for the 4-methyl tautomer and $378.4 \mathrm{~cm}^{-1}$ for the 5-methyl tautomer (see Tables 4.1,4.2).

In order to determine the relative abundance of the two tautomers in the gas mixture, the line strengths and frequencies from the RAM36 output were used to scale a simulated spectrum to the actual spectrum of each isomer. A least squares optimization was performed to scale the simulation and minimize the rms difference between the line center intensities of the simulation and the measured spectrum. For both tautomers the 
rms was calculated near $15 \%$, which is a typical relative intensity accuracy for the CP-FTmmW spectrometer. Across the band, 69 lines of 4-methylimidazole and 54 lines of 5-methylimidazole were used for the multi-point scaling. Only the strongest, well resolved lines representing a collapsed a/b quartet were used for matching. Since the 5methylimidazole A/E splitting was smaller there were fewer well resolved lines at low $\mathrm{K}_{\mathrm{a}}$ quantum number resulting in few lines for the matching compared to 4-methylimidazole. The results indicate a relative abundance of 3.23 to 1 in favor of the lower energy 4-methylimidazole tautomer of the analysis as displayed in Table 4.4. This translates to a relative Gibbs free energy difference of $242.8 \mathrm{~cm}^{-1}$ or $0.69 \mathrm{kcal} / \mathrm{mol}$.

Since RAM36 is a non-linear method, and strong correlations are expected between the parameters torsion parameters, a good initial guess is established for the rotational constants $\mathrm{A}, \mathrm{B}, \mathrm{C}$ and the quartic centrifugal distortion constants by fitting the A-state lines to a standard Watson A Hamiltonion using Picketts's SPFIT fitting program. The local A-state fits were comparable in rms to the $\mathrm{A} / \mathrm{E}$ ground state global fits both, well within the standards of accuracy for millimeter wave spectroscopy. In order to better define the torsional parameters, a global fit including the microwave and higher frequency submillimeter bands are required, however the millimeter wave line list is reported as supplementary material for the ground rotational state spectrum of the 4(5)-methylimidazole pair so that identifications can be made based in the $260-295 \mathrm{GHz}$ frequency band. [48] 


\begin{tabular}{|c|c|c|c|c|c|}
\hline Operator & Parameter & Value $\left(\mathrm{cm}^{-1}\right)^{\mathrm{a}}$ & Value $(v)^{\mathrm{a}}$ & $\begin{array}{l}\text { M062x } \\
6-311++g(d, p)^{b}\end{array}$ & 4-MI A state ${ }^{c}$ \\
\hline $\mathrm{p}_{\alpha}^{2}$ & $\mathrm{~F}$ & 5.38 & $160.1(\mathrm{GHz})$ & - & -- \\
\hline$(1 / 2)(1-\cos 3 \alpha)$ & $\mathrm{V}_{3}$ & $311.9362(78)$ & $935.16(24)(\mathrm{GHz})$ & $306.1(28)$ & -- \\
\hline $\mathrm{p}_{\alpha} \mathrm{P}_{\mathrm{a}}$ & $\rho$ (unitless) & $0.0563465(31)$ & $0.0563465(31)$ & 0.03006 & -- \\
\hline $\mathrm{P}^{2}{ }^{2}$ & $\mathrm{~A}_{\mathrm{RAM}}$ & $0.29908743(44)$ & 8966.416(13) (MHz) & $8972.570(\mathrm{MHz})$ & $8971.89831(109)(\mathrm{MHz})^{\mathrm{d}}$ \\
\hline $\mathrm{P}_{\mathrm{b}}^{2}$ & $\mathrm{~B}_{\mathrm{RAM}}$ & $0.11701867(45)$ & $3508.131(13)(\mathrm{MHz})$ & $3503.319(\mathrm{MHz})$ & $3508.27689(55)(\mathrm{MHz})^{\mathrm{d}}$ \\
\hline $\mathrm{P}_{\mathrm{c}}{ }^{2}$ & $\mathrm{C}_{\mathrm{RAM}}$ & $0.8543334(45)$ & $2561.227(13)(\mathrm{MHz})$ & $2559.499(\mathrm{MHz})$ & $2561.37076(37)(\mathrm{MHz})^{d}$ \\
\hline$-\mathrm{P}^{4}$ & $\Delta_{\mathrm{J}}$ & $0.83733(37) \times 10^{-8}$ & $0.25103(11)(\mathrm{kHz})$ & $0.241(\mathrm{kHz})$ & $0.251281(118)(\mathrm{kHz})$ \\
\hline$-\mathrm{P}^{2} \mathrm{P}_{\mathrm{a}}^{2}$ & $\Delta_{\mathrm{IK}}$ & $0.5324(28) \times 10^{-7}$ & $1.5961(84)(\mathrm{kHz})$ & $1.548(\mathrm{kHz})$ & $1.510469(261)(\mathrm{kHz})$ \\
\hline$-\mathrm{P}_{\mathrm{a}}^{4}$ & $\Delta_{\mathrm{K}}$ & $0.37481(27) \times 10^{-7}$ & $1.12364(80)(\mathrm{kHz})$ & $0.890(\mathrm{kHz})$ & $7.0055(48)(\mathrm{kHz})$ \\
\hline$-2 \mathrm{P}^{2}\left(\mathrm{P}_{\mathrm{b}}^{2}-\mathrm{P}_{\mathrm{c}}^{2}\right)$ & $\delta_{\mathrm{J}}$ & $0.22448(13) \times 10^{-7}$ & $0.067297(37) \mathrm{kHz}$ & $0.0653(\mathrm{kHz})$ & $0.0673866(314)(\mathrm{kHz})$ \\
\hline$-\left\{\mathrm{P}_{\mathrm{a}}^{2},\left(\mathrm{P}_{\mathrm{b}}^{2}-\mathrm{P}_{\mathrm{c}}^{2}\right)\right\}$ & $\delta_{\mathrm{K}}$ & $0.37368(39) \times 10^{-7}$ & $1.1203(12) \mathrm{kHz}$ & $0.382(\mathrm{kHz})$ & $0.58848(70)(\mathrm{kHz})$ \\
\hline \multirow[t]{8}{*}{$\mathrm{p}_{\alpha}^{2} \mathrm{P}^{2}$} & $\mathrm{~F}_{\mathrm{J}}$ & $0.2040(90) \times 10^{-5}$ & $0.0587(27)(\mathrm{MHz})$ & -- & - \\
\hline & $\Phi_{\mathrm{K}}$ & -- & -- & -- & $0.0673866(314)(\mathrm{Hz})$ \\
\hline & $\mu_{\mathrm{a}}(\mathrm{D})$ & & & 2.2 & \\
\hline & $\mu_{\mathrm{b}}(\mathrm{D})$ & & & 2.7 & \\
\hline & $\operatorname{rms} A(m=0)$ & & $0.0800(\mathrm{MHz})$ & & $0.0636(\mathrm{MHz})$ \\
\hline & $\operatorname{rms} \mathrm{E}(\mathrm{m}=1)$ & & $0.0563(\mathrm{MHz})$ & & \\
\hline & \# Lines A & 253 & 253 & & 267 \\
\hline & \# Lines E & 249 & 249 & & \\
\hline
\end{tabular}

a) results from a global A/E fit using RAM36, see reference 47

b) including anharmonic corrections

c) results from a local A state fit using SPFIT, see reference 45

d) principal axis 


\begin{tabular}{|c|c|c|c|c|c|}
\hline Operator & Parameter & Value $\left(\mathrm{cm}^{-1}\right)^{\mathrm{a}}$ & Value $(v)^{\mathrm{a}}$ & $\begin{array}{l}\text { M062x } \\
6-311++g(d, p)\end{array}$ & 5-MI A state \\
\hline $\mathrm{p}_{\alpha}{ }^{2}$ & $\mathrm{~F}$ & 5.5 & $164.8858519(\mathrm{GHz})$ & -- & -- \\
\hline$(1 / 2)(1-\cos 3 \alpha)$ & $\mathrm{V}_{3}$ & $378.416(68)$ & $1134.46(20)(\mathrm{GHz})$ & $364.2(70)$ & -- \\
\hline $\mathrm{p}_{\alpha} \mathrm{P}_{\mathrm{a}}$ & $\rho$ & $0.056292(28)$ & $0.056292(28)$ & 0.0535 & -- \\
\hline $\mathrm{P}_{\mathrm{a}}^{2}$ & $\mathrm{~A}_{\mathrm{RAM}}$ & $0.3020185(18)$ & $9054.286(55)(\mathrm{MHz})$ & $9067.579(\mathrm{MHz})$ & $9057.12732(292)(\mathrm{MHz})^{\mathrm{d}}$ \\
\hline $\mathrm{P}_{\mathrm{b}}^{2}$ & $\mathrm{~B}_{\mathrm{RAM}}$ & $0.1157839(19)$ & $3471.114(56)(\mathrm{MHz})$ & $3465.351(\mathrm{MHz})$ & $3471.61516(86)(\mathrm{MHz})^{\mathrm{d}}$ \\
\hline $\mathrm{P}_{\mathrm{c}}{ }^{2}$ & $\mathrm{C}_{\mathrm{RAM}}$ & $0.850148(19)$ & $2548.679(56)(\mathrm{MHz})$ & $2547.172(\mathrm{MHz})$ & $2549.44019(73)(\mathrm{MHz})^{\mathrm{d}}$ \\
\hline$-\mathrm{P}^{4}$ & $\Delta_{\mathrm{J}}$ & $0.84090(72) \times 10^{-8}$ & $0.25209(22)(\mathrm{kHz})$ & $0.242(\mathrm{kHz})$ & $0.252239(169)(\mathrm{kHz})$ \\
\hline$-\mathrm{P}^{2} \mathrm{P}_{\mathrm{a}}^{2}$ & $\Delta_{\mathrm{JK}}$ & $0.637(11) \times 10^{-7}$ & $1.908(32)(\mathrm{kHz})$ & $1.678(\mathrm{kHz})$ & $1.62400(49)(\mathrm{kHz})$ \\
\hline$-\mathrm{P}_{\mathrm{a}}^{4}$ & $\Delta_{\mathrm{K}}$ & $0.2420(26) \times 10^{-7}$ & $0.7254(77)(\mathrm{kHz})$ & $0.565(\mathrm{kHz})$ & $3.2455(160)(\mathrm{kHz})$ \\
\hline$-2 \mathrm{P}^{2}\left(\mathrm{P}_{\mathrm{b}}^{2}-\mathrm{P}_{\mathrm{c}}^{2}\right)$ & $\delta_{\mathrm{J}}$ & $0.22301(30) \times 10^{-8}$ & $0.066855(9)(\mathrm{kHz})$ & $0.0649(\mathrm{kHz})$ & $0.066857(73)(\mathrm{kHz})$ \\
\hline$-\left\{\mathrm{P}_{\mathrm{a}}^{2},\left(\mathrm{P}_{\mathrm{b}}^{2}-\mathrm{P}_{\mathrm{c}}^{2}\right)\right\}$ & $\delta_{\mathrm{K}}$ & $0.3931(7) \times 10^{-7}$ & $1.1785(21)(\mathrm{kHz})$ & $0.519(\mathrm{kHz})$ & $0.74739(176)(\mathrm{kHz})$ \\
\hline \multirow[t]{10}{*}{$\mathrm{p}_{\alpha}{ }^{2} \mathrm{P}^{2}$} & $\mathrm{~F}_{\mathrm{J}}$ & $0.376(33) \times 10^{-5}$ & $0.113(10)(\mathrm{MHz})$ & -- & -- \\
\hline & $\Phi_{\mathrm{K}}$ & -- & -- & & $0.9842(315)(\mathrm{kHz})$ \\
\hline & $\mu_{\mathrm{a}}(\mathrm{D})$ & & & 2.8 & \\
\hline & $\mu_{\mathrm{b}}(\mathrm{D})$ & & & 3.0 & \\
\hline & rms A & & & & \\
\hline & $(\mathrm{m}=0)$ & -- & 0.0827 & & $0.0729(\mathrm{MHz})$ \\
\hline & rms E & & & & \\
\hline & $(m=1)$ & -- & 0.0748 & & \\
\hline & \# Lines A & 189 & 189 & & 200 \\
\hline & \# Lines E & 112 & 112 & & \\
\hline
\end{tabular}

a) results from a global A/E fit using RAM36, see reference 47

b) including anharmonic corrections

c) results from a local A state fit using SPFIT, see reference 45

d) principal axis 


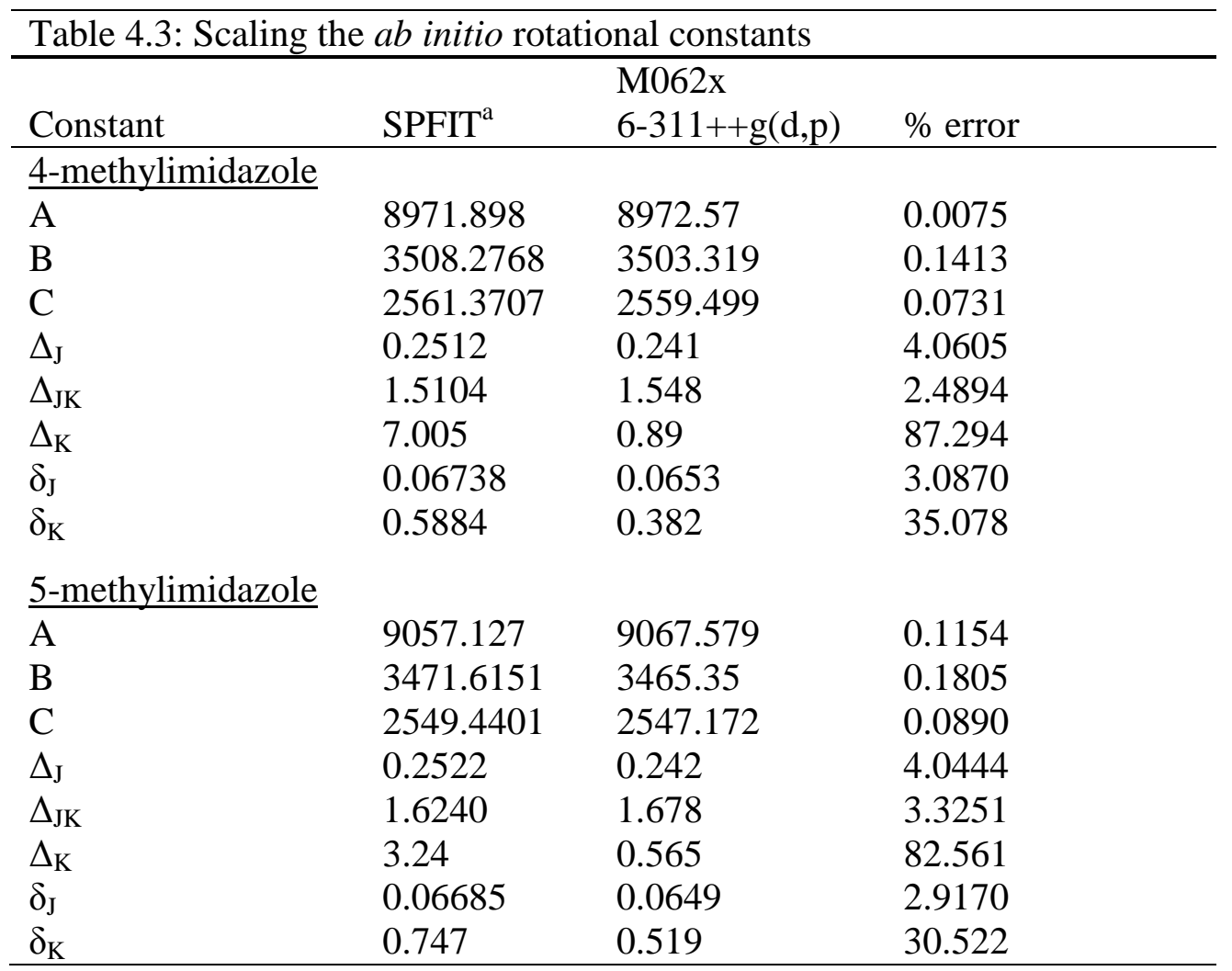

a) results from a local A state fit using SPFIT, see reference 45 
Table 4.4: 4(5)-methylimidazole relative abundance by CPFTmmW

\begin{tabular}{lll}
\hline & 4-methylimidazole & 5-methylimidazole \\
\hline \# lines & 69 & 54 \\
rms \% & 15.4 & 16.7 \\
average & -0.0025 & 0.0021 \\
scale & 1.241 & 0.384 \\
relative abundance & 3.23 & 1 \\
\hline
\end{tabular}




\section{Discussion}

The low pressure gas requirement for high-resolution rotational spectroscopy is an advantage that enables sampling flexibility. When dealing with liquids, headspace vapor is typically sufficient to produce the required number density (a few millitorr of partial pressure) for sensitive gas phase spectroscopy. For low vapor pressure liquids and solids, heating is typically used to enhance the vapor phase. The enhanced vapor pressure is necessary in order to continually supply the backing gas of a jet-expansion nozzle with sufficient analyte molecules in pulsed, microwave spectroscopy. The extreme hot to cold conditions, however, may negatively affect the sample. Although the conformational equilibrium can be frozen out and studied in the molecular jet, the appropriate equilibrium at some biological or atmospheric temperature of interest cannot be represented unless the backing gas is regulated at that temperature. This is straightforward to do, however, structures with low barriers between them can relax in the jet expansion and perturb the expected composition. [49]

The segmented chirped pulse spectrometer brings some advantages for measuring the headspace of solids without a jet expansion. Although a slow, steady flow was used in this work, static measurements also produced equivalently strong spectra. In a static 1L cell, assuming 1 mTorr partial pressure of 4(5)-methylimidazole, only $45 \mu \mathrm{mol}$ are required to make the measurement. Since the high dynamic range, CP-FTmmW full band spectrum takes just 3 minutes to acquire, even with a $1 \mathrm{~L} / \mathrm{min}$ flow only $135 \mu \mathrm{mol}$ of sample would be consumed. The low sample consumption relieves the need to heat the sample, which is advantageous for those that can degrade with moderate heating. For studying effects at a range of temperatures, the sample cell of the spectrometer can also 
be reasonably well controlled by using cooling or heating jackets. Since there is no jet expansion, the millimeter wave spectrum requires no carrier gas.

The tautomer equilibrium of 4(5)-methylimidazole has been studied both experimentally and computationally. In particular, Worth et al. 1989 used computational chemistry methods to calculate the gas phase equilibrium constant and Gibbs free energy. [50] These results illustrated a strong dependence of the values to converge towards accepted experimental values at higher levels of theory. However, experimental methods of measuring the tautomer equilibrium abundance had only been done in solution. Most involved aqueous solutions, however Schuster et. al. performed an analysis using $\mathrm{N}^{15}$ NMR with methylimidazole dissolved in several non-aqueous solvents. [51-55] The computational and experimental results for this CP-FTmmW study are compared to some non-aqueous historical results in Table 4.5. In order to generate more accurate $298 \mathrm{~K}$ thermochemical parameters, an MP2 level calculation with a 6-311++g(d,p) basis set was included in addition to the M06-2x result. The higher level calculations are in better agreement with experiment.

The CP-FTmmW experimental measurement is based on the abundance ratio determined from matching scaled simulations to experimental data that is influenced by the estimated dipole moments and experimental precision across the spectrometer band. CP-FTmmW measurements have reported to have a precision better than $1 \%$ and a broadband intensity accuracy of 15\%. [40] Since the intensity fluctuation across the band is random, the full band, multipoint comparison of 50-60 lines results in a statistically accurate relative abundance ratio. The scaling optimization for both tautomers resulted in an absolute average fluctuation about the simulated intensities of $0.002 \mathrm{mV}$, a $0.05 \%$ 
error for 4-methylimidazole signals and a $0.15 \%$ error for 5-methylimidazole signals. The largest source of error for the abundance determination comes from the error in the simulation resulting from the calculated dipole moments, not the CP-FTmmW spectrum. Dipole moment estimations are generally accurate to $10-20 \%$. Since signals in the weak pulse limit scale with $\mu^{2}$ this could translate to a $20-40 \%$ error in the simulated spectrum. Accurate experimentally measured dipole moments are the primary source to improve the error of the abundance determination reported in this work.

The 4-methylimidazole signal level is approximately $0.5 \mathrm{mV}$ against a 10,000 shot noise level of $2 \times 10^{-3} \mathrm{mV}$. Assuming the sqrt(N) signal averaging advantage, a 10 million shot noise level would be approximately $1 \times 10^{-5} \mathrm{mV}$. Precluding any reference spectrum, the total pressure may have contributions from air and water. The $0.5 \mathrm{mV}$ signal strength may represent a 1 mTorr partial pressure of 4-methylimidazole, for example, resulting in a $10 \mathrm{ppm}$ detection limit. By optimizing the detection for a specific subset of molecular lines, optimum $\pi / 2$ excitation can be used to both enhance the signal strength and decrease the measurement time. A $2 \mu$ s excitation/detection scheme with electronic background for a total of $4 \mu \mathrm{s}$ can be signal averaged 10 million times in $40 \mathrm{~s}$. The Henry's law constant of $4.1 \times 10^{-6} \mathrm{~atm} \mathrm{m^{3 }} / \mathrm{mol}$ suggests fractionation out of water will be very low so detection would favor direct liquid injection over headspace analysis. [41] The methylimidazole tautomers are relatively easy to distinguish in their equilibrium mixture using rotational spectroscopy; however, there has been some note of the limitations of microwave spectroscopy for conformer analysis in complex mixtures. For example, cytosine conformers were originally a challenge to independently assign in 


\begin{tabular}{lllllll}
\hline \multicolumn{2}{l}{ Table 4.5: 4(5)-methylimidazole gas equilibrium } & & & \\
\hline & Schuster 1979 & Worth 1989 & Charton 1965 & MP2 & M06-2X & \\
parameter & NMR (non-aq) & MP2/6-31G** & Hammett eqn & $16-311++\mathrm{g}(\mathrm{d}, \mathrm{p})^{\mathrm{a}}$ & $16-311++\mathrm{g}(\mathrm{d}, \mathrm{p})^{\mathrm{a}}$ & CP-FTmmW \\
\hline $\mathrm{kcal} / \mathrm{mol}$ & 0.8 & 0.33 & 0.6 & 0.525 & 0.432 & 0.694 \\
$\mathrm{~K}_{\mathrm{eq}}$ & 0.26 & 0.57 & 0.36 & 0.41 & 0.48 & 0.31 \\
$\Delta \mathrm{G}(\mathrm{KJ} / \mathrm{mol})$ & 3.35 & 1.38 & 2.51 & 2.20 & 1.81 & 2.90 \\
$\Delta \mathrm{G}(\mathrm{cm}-1)$ & 279.9 & 115.4 & 209.9 & 183.7 & 151.2 & 242.81 \\
$4-\mathrm{MI}:$ 5-MI & 3.86 & 1.75 & 2.75 & 2.43 & 2.07 & 3.22 \\
\hline
\end{tabular}

a) Includes zero point energy corrections 
the microwave spectrum. [10,14] Today, microwave studies can be revived with a combination of much higher level computational chemistry and new time domain CP-FT spectrometers that can implement double resonant techniques in order verify conformer assignment through probing the rotational energy manifold. The capability is applicable across a broader context of synthesized mixtures that require in situ chemical tests for identity verification.

In addition to cataloguing the rotational signature of the 4(5)-methylimidazole tautomers, the results have implications to industrial scale analytical chemistry. The speed of CP-FTmmW is presented as an advantage for low sample consumption important for measuring the headspace of solids. In addition to chemical identification of the solid, the spectrometer can be used as a detector in combination with thermo gravimetric analysis of solids as means to analyze solvent content. Without recrystallization, the appearance of acetic acid in the headspace of 4(5)-methylimidazole solid demonstrated a concept for residual solvent analysis, a very high throughput assay used in pharmaceutical manufacturing and a production bottleneck using the current chromatographic methods. [56]

\section{Conclusion}

We have presented the first published rotational spectrum of methylimidazole isomers and the first results using a segmented CP-FTmmW spectrometer for millimeter wave measurements of new molecules. One of the motivations behind new spectrometer designs in rotational spectroscopy is to transform the laboratory-made, research specific image into one that looks and works like a commercial instrument for analytical 
chemistry. The 4(5)-methylimidazole mixture was chosen as an analytical challenge that requires the high chemical selectivity of rotational spectroscopy; the sampling benefits of CP-FTmmW, and comes with a pressing need for detection methods. The 4(5)-methylimidazole tautameric pair is a mixture that cannot be conventionally separated by chemical means and since the analysis requires absolute structure selectivity only a few methods can answer the analytical challenge. Rotational spectra are ideal for structure determination because of the well-understood direct connection between the molecular mass distribution and the rotational energy levels. 4-methylimidazole is the only regulated methylimidazole isomer and requires chemical separation in a GC-MS detection in order to distinguish it from other unregulated methylimidazole isomers. Detection by their rotational spectrum would circumvent the need for chromatography.

Chirped pulse spectroscopy has become an important analytical tool in rotational spectroscopy because it has changed the analysis approach from one of confirmation and refinement to one of unbiased investigation. The broader scanning capability of millimeter/submillimeter spectroscopy is one of the advantages that make it complementary to the higher resolution of microwave spectroscopy. Now, fast, full band data sets can be generated up to $300 \mathrm{GHz}$ using time domain chirped pulse spectroscopy. The extension of chirped pulse rotational spectroscopy to millimeter/submillimeter wavelengths is a step forward toward the day when a spectroscopist can chose the best spectral region based solely on the molecular dynamic of interest as opposed to technology limitations. 


\section{References for Chapter 4:}

1. B. Pullman, A. Pullman, Advan. Heterocycl. Chem., 1977, 13, 77.

2. R. Stolarski, B. Kierdaszuk, C. E. Hagberg, D. Shugar, Biochem., 1984, 23, 2906.

3. Y. Podolyan, L. Gorb, J. Lesczynski, Int. J. Mol. Sci., 2003, 4, 410.

4. J. D. Watson, F. H. C. Crick, Nature, 1953, 171, 737.

5. L. Zandee, R. B. Bernstein, J. Chem. Phys., 1979, 71, 1359.

6. C. Plutzer, I. Hunig, K. Kleinermanns, Phys. Chem. Chem. Phys., 2003, 5, 1158.

7. J. R. Carney, T. S. Zweir, J. chem. Phys., 2000, 104, 8677.

8. E. Nir, P. Imhof, K. Kleinermanns, M. S. de Vries, J. Am. Chem. Soc., 2000, 122, 8091.

9. E. Nir, C. Janzen, P. Imhof, K. Kleinermanns, M. S. de Vries, J. Chem. Phys., 2001, 115, 4604

10. R. D. Brown, P. D. Godfrey, D. McNaughton, A. P. Pierlot, J. Am. Chem. Soc., 1989, 89, 2308.

11. B. Vogelsanger, P. D. Godfrey, R. D. Brown, J. Am. Chem Soc., 1991, 113, 7864.

12. R. D. Brown, P. D. Godfrey, D. McNaughton, A. P. Pierlot, Chem. Phys. Lett., 1989, 156, 61 .

13. S. Samdal, H. Mollenal, J. Chem. Phys., 2011, 115, 7559.

14. M. Y. Choi, F. Dong, R. E. Miller, Phil. Trans. R. Soc. A, 2005, 363, 393.

15. M. Y. Choi, R. E. Miller, J. Am. Chem. Soc., 2006, 128, 7320.

16. P. D. Godfrey, E. G. Robertson, J. Chem. Phys., 2012, 137, 064306.

17. G. L. Blackman, R. D. Brown, F. R. Burden, I. R. Elsum, J. Mol. Spec., 1976, 60, 63.

18. NIH. National Toxicology Program (NTP) (2004b) NTP technical report on the toxicology and carcinogenesis studies of 2-methylimidazole (CAS No. 693-98-1) in F344/N rats and B6C3F1 mice (feed studies). Technical report series no. 516. NIH Publication No. 05-4456. U.S. Department of Health and Human Services, Public Health Service, National Institutes of Health, Research Triangle Park, NC. 
19. NIH. National Toxicology Program (NTP) (2006) NTP technical report on the toxicology and carcinogenesis studies of 4-mmethylimidazole (CAS No. 822-36-6) in F344/N rats and B6C3F1 mice (feed studies). Technical report series No. 535. NIH Publication No. 05- 4471. U.S. Department of Health and Human Services, Public Health Service, National Institutes of Health, Research Triangle Park, NC.

20. P. Chan, J. Mahler, G. Travlos, A. Nyska, M. Wenk, Arch. Toxicol., 2006, 80, 169.

21. P. C. Chan, G. D. Hills, G. E. Kissling, A. Nyska, Arch. Toxicol., 2008, 82, 45.

22. J. K. Moon, T. Shibamoto, J. Agric. Food. Chem., 2011, 59, 615.

23. B. Klejdus, J. Moravcova, L. Lojkova , J. Vacek, V. Kuban, J. Sep. Sci., 2006, 29, 378.

24. L. Lojkova, B. Klejdus, J. Moravcova, V. Kuban, Food Addit. Contam. 2006, 23, 963

25. S. Casal, J. O. Fermandes, M. B. P. P. Oliveira, M. A. Ferreira, J. Chromatogr., A, 2002, 975, 285.

26. S. Cunha, A. I. Barrado, M. A. Faria, J. O. Fernandes, J. Food Comp. Anal., 2011, 24, 609.

27. J. F. da Silveira Petruci, E. A. Pereira, A. A. Cardoso, J. Agric. Food. Chem., 2013, 61, 2263.

28. C. Shlee, M. Markova, J. Schrank, F. Laplagne, R. Shneider, D. W. Lachenmeier, J. Chrom. B, 2013, 927, 223.

29. Office of Environmental Health Assessment (OEHHA).

30. K. Hasegawa, T. Ono, T. Noguchi, J. Phys. Chem. B, 2000, 104, 4265.

31. L. Klasinc, B. Ruscic, F. Kajfez, V. Sunjic, International J. Quant. Chem.: Quantum Biology Symposium, 1978, 5, 367.

32. H. W. Harrington, J. R. Hearn, R. F. Rauskolb, Hewlett-Packard Journal, 1971, 22, 1.

33. R. D. Suenram, J. U. Grabow, A. Zuban, I. Leanov, Rev. Sci. Instru., 1999, 70, 2127.

34. M. D. Harmony, K. A. Beran, D. M. Angst, K. L. Ratzlaff, Rev. Sci. Instru., 1995, 66, 5196.

35. I. R. Medvedev, C. F. Neese, G. M. Plummer, F. C. De Lucia, Opt. Lett., 2010, 35, 1533. 
36. C. Neese, I. Medvedev, G. Plummer, A. Frank, C. Ball, F. De Lucia, IEEE Sensors J., 2012, 12, 2565.

37. S. Albert, D. T. Petkie, R. P. A. Bettens, S. P. Belov, F. C. De Lucia, Anal. Chem. 1998, 70, 719A.

38. I. R. Medvedev, M. Behnke, F. C. De Lucia, Analyst, 2006, 131, 1299.

39. J. L. Niell, B. J. Harris, A. L. Steber, K. O. Douglas, D. F. Plusquellic, B. H. Pate, Opt. Expres., 2013, 21, 19743.

40. B. J. Harris, A. L. Steber, B. H. Pate, Rev. Sci. Instr., in preparation.

41. USEPA. Estimation Program Interface (EPI) Suite, ver. 4.0. available from: http://www.epa.gov/oppt/exposure/pubs/episuitedl.htm.

42. M. J. Frish et al., Gaussian 09, Gaussian Inc., Wallingford CT, 2009.

43. D. F. Plusquellic, R. D. Suenram, B. Mate, J. O. Jenson, A. C. Samuels, J. Chem. Phys. 2001, 115, 3057. The JB95 spectral fitting program can be downloaded from the following web address: http://www.nist.gov/pml/div685/grp08/jb95.cfm.

44. H .M. Pickett, J. Mol. Spectrosc., 1991 148, 371.

45. Z. Kisiel, L. Pszczolkowski, I. R. Medvedev, M. Winnewisser, F. C. De Lucia, E. Herbst, J.Mol.Spectrosc., 2005, 233,231.

46. V. V. Ilyushin, Z. Kisieal, L. Pszczolowski, H. Mader, J. T. Hougen, J. Mol. Spec., 2010, 259, 26.

47. V. V. Illyushin, C. P. Endres, F. Lewen, S. Schlemmer, B. J. Drouin, J. Mol. Spec., 2013, 290, 32.

48. supplementary material.

49. R. S. Ruoff, T. D. Klots, T. Emilsson, H. S. Gutowsky, J. Chem. Phys., 1990, 93, 3142.

50. G. A. Worth, P. M. King, W. G. Richards, Biochimica et Biophysica Acta, 1989, 993, 134.

51. J. Catalan, J. L. G. de Paz, M. Yanez, R. M. Claramunt, C. Lopez, J. Elguero, F. Anvia, J. H. Quian, M. Taagepera, R. W. Taft, JACS, 1990, 112, 1303. 
52 F. J. Luque, J M. Lopez-Bes, J. Cemeli, M. Aroztegui, M. Orozco, Thero. Chem. Acc., 1997, 96, 105.

53 G. S. Li, M. F. Ruiz-Lopez, B. Maigret, J. Phys. Chem. A, 1997, 101, 7885.

54. M. Charton, J. Org. Chem., 1965, 30, 3346.

55. I. I. Schuster, J. D. Roberts, J. Org. Chem., 1979, 44, 3865.

56. USP-NF. General Chapter 467: Residual Solvents, 2007. 


\section{Concluding Chapter: A New Generation for Rotational Spectroscopy}

This dissertation has thus far highlighted the fundamental advantages of chirped pulse Fourier transform instruments for millimeter/submillimeter spectroscopy (CP-FTmmW). The broadband, multiplexing approach translates the high power output of new millimeter light sources directly into sensitivity enhancement, a concept inaccessible for absorption spectrometers. The time resolved excitation/detection approach also brings new measurement modes that are challenging for direct absorption spectroscopy, which must rely on detection against a strong excitation light field. Double resonance methods for identity verification and free induction decay modeling for mass estimation can enhance the CP-FTmmW measurement with information to aid in spectral assignment.

The real-time segmented CP-FTmmW spectrometer relies on the convergence of two modern technical developments: new solid-state active multiplier chains for generating broadband, high frequency light ( > $200 \mathrm{GHz}$ ) and advances in high-speed digital electronics. Both fields are continually developing the next generation products to answer the demands of an expanding market. For a new technology like CP-FTmmW, it is important to have external drivers commoditizing the underlying hardware. The defense and communications industries are substantial contributors to the advancement of millimeter/submillimeter technology making the spectrometer design a systems integration problem rather than a much slower moving, basic engineering design

problem. The only customized components in the CP-FTmmW spectrometer are the AMCs, which require a choice of bandwidth window to concentrate all of the power. It is 
very conceivable that this cryogen free, software controlled spectrometer could be manufactured to scale as a commercial product line.

The basics of performance and cost are in a healthy balance. The CP-FTmmW spectrometer is a competitive design compared to its direct counterparts in rotational spectroscopy and is a strong design for a commercially viable instrument, however, the value must be validated against the current suite of analytical instrumentation; it should be validated by analytical chemists themselves. The National Science Foundation gives academic researchers the opportunity to simulate a start-up company and validate the value of their new technology in front of would be users though the Innovation Corps ( $\mathrm{i}$-Corps) program. Over the course of nearly 100 interviews in the i-Corps program, the CP-FTmmW spectrometer was placed into the landscape of analytical chemistry to learn about the challenges that are not well addressed by current techniques.

The life sciences and chemical instrumentation sector is a $\$ 45$ billion market, where spectroscopy (including mass spectrometry) takes up a substantial portion of $\$ 15$ billion and gas detection equipment occupies $\$ 1.5$ billion of the spectroscopy share. New instruments for gas detection are being marketed for monitoring airborne molecular contaminants in semiconductor cleanrooms, toxic chemicals in work environments, and volatiles content in chemical manufacturing. The fundamental advantage of rotational spectroscopy for gas mixture analysis has already been highlighted throughout this dissertation as absolute chemical selectivity. Other metrics are compared in Table 5.1. This is the kind of analysis that a technical executive must consider as he/she weighs the total cost of ownership which includes challenges to method transfer, throughput, training level, and maintenance. 
Table 5.1: The instrumentation landscape for gas mixture analysis

\begin{tabular}{ccccc}
\hline & CP-FTmmW & GC-FID/MS & FTIR & NIR (CRDS) \\
\hline Coverage & High & High & High & Low \\
Sensitivity & High & High & Low & High \\
Throughput & High & Low & Medium & High \\
Cost & $\mathbf{\$ 3 0 k - 5 0 0 k}$ & \$20k-500k & \$20k-80k & \$20k-70k \\
User & Technician & Scientist & Technician & Technician \\
Form Factor & Benchtop & Benchtop & Bench/Handheld & Rackmount \\
Maturity & New & 50+ years & 50+ years & 10 years \\
\hline
\end{tabular}

Value proposition for CP-FTmmW for each method

1) GC-FID or GC/MS: For current users of gas chromatography-flame ionization detector (GC-FID) or gas chromatography-mass spectroscopy (GC-MS) instruments, CP-FTmmW can deliver similar sensitivity and selectivity for VOC mixture analyses, but the advantages for CP-FTmmW are the simplification or elimination of the required sample handling, reduction in operator expertise and training required, increased sample throughput (analysis time), and reduced ongoing costs of consumables.

2) FTIR: For Fourier transform-infrared (FTIR) users, CP-FTmmW offers vastly superior spectral resolution so that complex mixtures can be analyzed with the same instrument footprint and simple sampling methodology. CP-FTmmW can achieve measurement dynamic ranges of $10^{5}$ or higher and significantly outperforms FT-IR spectroscopy.

3) NIR laser absorption and CRDS: CP-FTmmW instruments can offer similar sensitivity to cavity ringdown spectroscopy (CRDS) instruments, but without the requirement of buying a separate instrument for each molecule to be monitored. 
One CP-FTmmW instrument can, therefore, replace a large number of CRDS instruments.

There are significant opportunities for improving throughput in applications that require column chromatography; for example, residual solvent analysis in the pharmaceutical industry. The speed and simplicity of CP-FTmmW are very attractive to analytical chemists. However, as a fundamentally new detection principle for analytical chemistry, it also brings new capabilities that are derived from the absolute molecular structure selectivity. For example, isotopic ratio analysis can be redefined using rotational spectroscopy, which can identify the site of isotopic enhancement on a molecule, because each isotopologue has a distinctly resolved spectrum. 2D compound specific isotope analysis is becoming a popular technique, which involves monitoring the

isotope fractionation of two elements at once, for example, ${ }^{13} \mathrm{C} /{ }^{12} \mathrm{C}$ and ${ }^{15} \mathrm{~N} /{ }^{14} \mathrm{~N}$. Thus, it may be appropriate to market rotational spectrometers as a $3 D$ isotopic fractionation analyzer.

\section{Answering the needs for laboratory astrochemistry:}

As described in the introductory chapter, the interstellar medium (ISM) has a large chemical inventory of species considered to be high-energy, transient intermediates by terrestrial standards. This truth has driven the development of new synthetic methods, many of which are paired with millimeter/submillimeter spectrometers. The advantage of chirped pulse spectroscopy is the speed at which one can capture a sufficiently broad band of data at high dynamic range and produce a spectrum with unbiased chemical coverage. Although there is some informed intuition involved in targeting a molecule for 
discovery in the ISM, the complete understanding of the complex conditions under which molecules are formed is a challenge, and there is a large effort towards generating broad molecular line surveys of molecular clouds and circumstellar envelopes. The same concept should be true for laboratory measurements. By generating a broad spectral view of the complex laboratory chemistry, discovery can be focused on features that match the broad molecular line surveys of the ISM (representing the complex chemistry of the molecular cloud). This concept was the motivation behind the "reaction screening" approach employed in recent CP-FTMW studies and introduced earlier by Miller et al. in the $1950 \mathrm{~s}$ to investigate the production of pre-biotic molecules from simple carbon-oxygen-nitrogen parent molecules. [1-4]

Astrochemists should view chirped pulse spectroscopy as a tool for visualizing the broad chemical composition that ensues from conventional synthetic methods involving electric discharge, laser ablation, laser photolysis, etc. Since the time scale for broadband chirped pulse is on the order of microseconds and a typical radical or intermediate has a lifetime of up to several milliseconds after an excitation pulse (excimer laser or mercury flash), the CP-FTmmW spectrometer can capture the evolution of several chemical species at once to monitor the multiple branching ratios that result in a complex reaction. [5-7] These studies would involve introducing the energy source to a reactant gas flow. A custom gas mixture consisting of many proposed parent molecules could be introduced in a gas flow and coupled with electric discharge excitation or excimer laser excitation. The experiment would pose a challenge to identifying all of the daughter species, but matches could be made to known libraries and give astrochemists an indication of which kind of excitation techniques or source materials are best for 
matching the composition of a particular source in the ISM. For example, there are general distinctions in molecular clouds: oxygen rich regions versus nitrogen rich regions. [8] The same is true for circumstellar envelopes where carbon rich, oxygen rich, or even nitrogen rich (indicated for WR stage stars) content can strongly influence the chemical composition. $[9,10]$

In some cases, an experiment run time can be limited by the deposition of soot in the measurement cell or the depletion of reactant (when melting solids, for example). Faster spectroscopy can be a benefit when reactant materials are expensive or a challenge to introduce at sufficient number density. Metal bearing molecules are particularly challenging to produce, especially for high boiling point metals like titanium; however, metals are known to be a significant contributor to the molecular inventory of the circumstellar envelopes. [11-14] Broad spectral searching in the laboratory can necessitate multiple measurement runs with new solid sample and a clean spectrometer. The speed of chirped-pulse spectroscopy can turn a month's experiment into a day's work. Due to the challenges in laboratory synthesis, the search for metal-bearing molecules would benefit from the reaction screening approach, so that discovery can be focused on the readily produced, more abundant molecules in the reaction mixture. The screening approach could be applied to the study of metal-carbon chain formation, for example. Saturated carbon chains are abundant in the carbon rich circumstellar shell of IRC+102016. [15] It is possible that some metal content is tied up in Metal- $\mathrm{C}_{2 \mathrm{n}+1} \mathrm{H}$, Metal- $\mathrm{C}_{2 \mathrm{n}+1} \mathrm{~N}$ or Metal- $\mathrm{C}_{\mathrm{n}}$ species, of which there are many candidates for detection. [16-18] The fast, high dynamic range, broad band capabilities of the CP-FTmmW 
spectrometer is ideal for investigating the many reaction products that may form with acetylene, for example, in the presence of electric discharge and metal vapor. $[19,20]$

Although modern direct absorption spectrometers can introduce a significant speed advantage over many spectrometers currently in astrochemistry laboratories, they are not well suited for the advanced analytical tools presented in Chapter 2. Due to their transient nature, for many astrochemically relevant molecules, chemical identification cannot be made based off of a pure reference spectrum. Identification is usually verified by indirect chemical tests (removing a reactant, for example) and by fitting to a rotational Hamiltonian consistent with the molecule's structure. It is also generally true that new molecule detections will be increasingly focused on the weaker signals in molecular line surveys. These species are likely to pose a challenge for confident identification in laboratory synthesis, as well. Since they must be identified in the presence of a laboratory mixture, the advanced analytical tools presented in Chapter 2 can be used to speed spectral assignment. Double resonance identity verification and mass estimation may prove to be an important test for identifying diatomic or linear molecules, which do not have the benefit of high spectral redundancy. 2D double resonance can also be used to find spectral connections.

In summary, the CP-FTmmW broadband spectrometer speed and advanced measurement modes can bring a significant enhancement to molecule discovery in Astrochemistry studies. The design is accomplished in the millimeter light generation and detection, with the result that CP-FTmmW can be coupled without modification to current measurement cells and synthetic techniques. In fact, for applying the benefits of FT emission spectroscopy to narrow-band monitoring, and for some of the advanced 
analysis tools, a simple, low-cost "upgrade" could be designed as an add-on to current absorption spectrometers. Chirped-pulse spectrometers are already becoming a mainstream for new instruments in microwave spectroscopy. [21-28] The adoption of CP-FTmmW is a likely extension to that trend.

\section{Conclusion:}

The CP-FTmmW spectrometer is tool that can finally deliver the power of rotational spectroscopy to a broader scientific community. For physical chemistry research, the speed enables time resolved analysis for studying transient phenomena in dynamic spectroscopy or for studying reaction kinetics. For astrochemistry, the broadband investigative power, and the analytical tools can speed new molecule discovery. In general, the speed, broad band chemical coverage, and software controlled customizability of CP-FTmmW will give scientists in these fields the opportunity to focus more of their attention and time on the actual data which is the window to new discovery rather than constantly grappling with a tool that can frequently be a barrier to progress. The same concept is the principle behind new instruments in analytical chemistry. Historically, there has been a blind spot in the analytical chemistry suite-the frequency gap in the electromagnetic spectrum from microwave to terahertz wavelengths. This gap has been an oversight by analytical chemists for so long that some do not even realize it exists. With the introduction of CP-FTmmW, rotational spectroscopy can finally bring light to that frequency gap. 


\section{References for Concluding Chapter:}

1. D.P. Zaleski, N. A. Seifert, A.L. Steber, M.T. Muckle, R. A. Loomis, J.F. Corby, O. Martinez, K.N. Crabtree, P.R. Jewell, J.M. Hollis, F.J. Lovas, D. Vasquez, J. Nyiramahirwe, N. Sciortino, K. Johnson, M.C. McCarthy, A.J. Remijan, B.H. Pate, ApJ, 2013, 765, L10.

2. R. A. Loomis, D.P. Zaleski, A.L. Steber, J.L. Neill, M.T. Muckle, B.J. Harris, J.M. Hollis, P.R. Jewell, V. Lattanzi, F.J. Lovas, O. Martinez, M.C. McCarthy, A.J. Remijan, B.H. Pate, and J.F. Corby, ApJ, 2013, 765, L9.

3. S. L. Miller, Sci., 1953 117, 528.

4. S. L. Miller, H. C. Urey, Sci., 1959, 130, 245.

5. Y. Endo, H. Kanamori, E. Hirota, Laser Chem., 1987, 7, 61.

6. W. F. Kolbe, B. Leskovar, Rev. Sci. Instrum., 1985, 56, 1577.

7. S. Saito, J. Chem. Phys., 1970, 53, 2544.

8. D. N. Friedel, L. E. Snyder, ApJ, 2008, 672, 962.

9. E. D. Tenenbaum, J. L. Dodd, S. N. Milam, N. J. Woolf, L.M. Ziurys, ApJ, 2010, 720, L102.

10. J. R. Rizzo, J. Martin-Pintado, C. Henkel, ApJ, 2001, 553, L181.

11. K. I. Namiki, S. Saito, J. C. Robinson, T. C. Steimle, J. Mol. Spec., 1988, 191, 176.

12. S. Brunken, H. S. P. Muller, K. M. Menten, M. C. McCarthy, P. Thaddeus, ApJ, 2008, 676, 1367.

13. T. Kaminski, C. A. Gottleib, K. M. Menten, N. A. Patel, K. H. Young, S. Brunken, H. S. P. Muller, M. C. McCarthy, J. M Winters, L. Decin, A\&A, 2013, 551, A113.

14. R. L Pulliam, L. N. Zack, L. M. Ziurys, J. Mol. Spec., 2010, 264, 50.

15. E. D. Tenenbaum, J. L. Dodd, S. N. Milam, N. J. Woolf, L.M. Ziurys, ApJ Lett., 2010, 190, 348 .

16. S. Petrie, MNRAS, 1999, 302, 482.

17. R. C. Dunbar, S. Petrie, ApJ, 2002, 564, 792.

18. A. Largo, P. Redondo, C. Barrientos, J. Am. Chem. Soc., 2004, 126, 14611. 
19. J. Min, D. T. Halfen, M. Sun, B. Harris, L. M. Ziurys, J. Chem. Phys., 2010, 136, 244310 .

20. M. Sun, D. T. Halfen, J. Min, B. Harris, D. J. Clouthier, L. M. Ziurys, J. Chem. Phys. 2010, 133, 174301.

21. G. G. Brown, B. C. Dian, K. O. Douglass, S. M. Geyer, B. H. Pate, J. Mol. Spec., 2006, 238, 200.

22. G. G. Brown, B. C. Dian, K. O. Douglass, S. M. Geyer, S. T. Shipman, B. H. Pate, Rev. Sci. Instr., 2008, 79, 053103.

23. D. Zaleski, J. Neill, M. Muckle, N. Seifert, P. Carroll, S. Widicus-Weaver, B. Pate, J. Mol. Spec., 2012, 280, 68.

24. G. S. Grubbs, C. T. Dewberry, K. C. Etchison, K. E. Kerr, S. A. Cooke, Rev. Sci. Instr., 2007, 78, 096106.

25. S. L. Stevens, N. R. Walker J. Mol. Spec., 2010, 263, 27.

26. D. A. Obenchain, A. A. Elliott, A. L. Steber, R. A. Peebles, S. A. Peebles, C. J. Wurrey, G. A. Guirgis, J. Mol. Spec., 2010, 261, 35.

27. R. G. Bird, J. L. Neill, V. J. Alstadt, J. W. Young, B. H. Pate, D.W. Pratt, J. Phys. Chem. A, 2011, 115, 9392.

28. G. B. Park, A. H. Steeves, K. Kuyanov-Prozument, J. L. Neill, R. W. Field, J. Chem. Phys., 2011, 135, 024202. 